\title{
Study of $B_{s} \rightarrow K^{(*)} \mathscr{\ell}^{+} \mathscr{\ell}^{-}$decays in the PQCD factorization approach with lattice QCD input
}

\author{
Su-Ping Jin, ${ }^{1, *}$ Xue-Qing Hu $\odot,{ }^{1, \dagger}$ and Zhen-Jun Xiao $\odot^{1,2, \$}$ \\ ${ }^{1}$ Department of Physics and Institute of Theoretical Physics, Nanjing Normal University, \\ Nanjing, Jiangsu 210023, People's Republic of China \\ ${ }^{2}$ Jiangsu Key Laboratory for Numerical Simulation of Large Scale Complex Systems, \\ Nanjing Normal University, Nanjing 210023, People's Republic of China
}

(Received 31 March 2020; accepted 22 June 2020; published 7 July 2020)

\begin{abstract}
In this paper, we studied systematically the semileptonic decays $\bar{B}_{s} \rightarrow K^{(*)} \ell^{+} \ell^{-}$with $l^{-}=\left(e^{-}, \mu^{-}, \tau^{-}\right)$ by using the perturbative QCD (PQCD) and the "PQCD + Lattice" factorization approach, respectively. We first evaluated all relevant form factors $F_{i}\left(q^{2}\right)$ in the low- $q^{2}$ region using the PQCD approach, and we also took the available lattice QCD results at the end point $q_{\max }^{2}$ as additional inputs to improve the extrapolation of the form factors to the high- $q^{2}$ region. We calculated the branching ratios and twelve other kinds of physical observables. From our studies, we find the following points: (a) for $\bar{B}_{s} \rightarrow K l^{+} l^{-}$decays, the PQCD and PQCD + Lattice predictions for branching ratios $\mathcal{B}\left(\bar{B}_{s} \rightarrow K l^{+} l^{-}\right)$, the ratios of the branching ratios $R_{K}^{e \mu}$ and $R_{K}^{\mu \tau}$, and the longitudinal polarization asymmetry of the leptons $P_{L}$ agree well within errors; (b) the PQCD and PQCD + Lattice predictions for the $C P$-averaged branching ratio $\mathcal{B}\left(\bar{B}_{s} \rightarrow\right.$ $\left.K^{*} \mu^{+} \mu^{-}\right)$are $\left(3.17_{-0.78}^{+0.95}\right) \times 10^{-8}$ and $\left(2.48_{-0.50}^{+0.56}\right) \times 10^{-8}$ respectively, which agree well with the $\mathrm{LHCb}$ measured value $(2.9 \pm 1.1) \times 10^{-8}$ and the light-cone sum rule prediction; (c) for the ratios $R_{K^{*}}^{e \mu}$ and $R_{K^{*}}^{\mu \tau}$, the PQCD and PQCD + Lattice predictions agree well with each other and have a small error less than $10 \%$; (d) for the direct $C P$ asymmetries $\mathcal{A}_{C P}$ of all considered decay modes, they are always very small as expected: less than $5 \%$ in magnitude; (e) for the angular observables $P_{1,2,3}$ and $P_{4,5,6,8}^{\prime}$, our theoretical predictions for each kind of lepton are consistent within errors; (f) the theoretical predictions of the angular observables $P_{3}$ and $P_{6}^{\prime}$ are less than $10^{-2}$ in size, but the magnitude of $P_{1,2}$ and $P_{4,5}^{\prime}$ are larger than 0.2 ; and (g) the PQCD and PQCD + Lattice predictions of the binned values of all considered observables in the two $q^{2}$ bins [0.1-0.98] $\mathrm{GeV}^{2}$ and $[1.1-6] \mathrm{GeV}^{2}$ generally agree with each other and are also consistent with the light-cone sum rule results within errors. We believe that above predictions could be tested by future $\mathrm{LHCb}$ and Belle-II experiments.
\end{abstract}

DOI: 10.1103/PhysRevD.102.013001

\section{INTRODUCTION}

The lepton flavor universality (LFU), as one of the distinctive hypotheses of the standard model (SM), requires the same kinds of couplings between the gauge bosons and the three families of leptons except for mass effects. However, the recently reported $R_{K}$ and $R_{K^{*}}$ anomalies bring a primary hint of the LFU violation. The measured values of the ratios $R_{K}$ and $R_{K^{*}}$, defined as the ratios of the

\footnotetext{
*2223919088@qq.com

†hu-xueqing@qq.com

xiaozhenjun@njnu.edu.cn
}

Published by the American Physical Society under the terms of the Creative Commons Attribution 4.0 International license. Further distribution of this work must maintain attribution to the author(s) and the published article's title, journal citation, and DOI. Funded by SCOAP ${ }^{3}$. branching fractions (BRs) $\mathcal{B}\left(B \rightarrow K^{(*)} \mu^{+} \mu^{-}\right)$and $\mathcal{B}(B \rightarrow$ $K^{(*)} e^{+} e^{-}$) [1], are clearly smaller than the SM predictions [2-8]: the deviation is about $2.6 \sigma$ for $R_{K}$ and $2.3 \sigma$ for $R_{K^{*}}$ [9-14]. In addition, the LHCb experiment first observed the so-called $P_{5}^{\prime}$ anomaly, a sizeable discrepancy at $3.7 \sigma$ between the measurement and the SM prediction in one bin for the angular observables $P_{5}^{\prime}[15,16]$.

If the above mentioned anomalies are indeed the signal of the LFU violation in $b \rightarrow s \ell^{+} \ell^{-}$decays, it must appear in the similar process $b \rightarrow d \ell^{+} \ell^{-}$, because they are the same kinds of flavor-changing neutral current (FCNC) transitions at the quark level with the differences of Cabibbo-Kobayashi-Maskawa (CKM) matrix elements $\left(V_{t d}\right.$ vs $\left.V_{t s}\right)$ and the masses $\left(m_{d}\right.$ vs $\left.m_{s}\right)$. As a consequence of the Glashow-Iliopoulos-Maiani mechanism [17], the flavor structure of the SM theory permits the FCNC to arise at the loop level only, leaving some space for heavy new degrees of freedom to contribute to these rare 
processes [18]. With the same quark level $b \rightarrow d \mu^{+} \mu^{-}$ transitions, the exclusive $B^{ \pm} \rightarrow \pi^{ \pm} \mu^{+} \mu^{-}$and $B_{s}^{0} \rightarrow$ $\bar{K}^{* 0} \mu^{+} \mu^{-}$decays have been measured recently by the LHCb experiment $[19,20]$ :

$$
\begin{aligned}
& \mathcal{B}\left(B^{ \pm} \rightarrow \pi^{ \pm} \mu^{+} \mu^{-}\right)=(1.83 \pm 0.24 \text { (stat. }) \\
& \pm 0.05(\text { syst. })) \times 10^{-8}, \\
& \mathcal{B}\left(B_{s}^{0} \rightarrow \bar{K}^{* 0} \mu^{+} \mu^{-}\right)=(2.9 \pm 1.0 \text { (stat.) } \pm 0.2 \text { (syst. }) \\
& \pm 0.3(\text { norm. })) \times 10^{-8},
\end{aligned}
$$

and they agree well with those currently available SM predictions as given, for example, in Refs. [21-29].

In this paper, $K^{* 0}$ denotes a vector $K^{* 0}(892)$ meson, which is reconstructed in the $K^{+} \pi^{-}$final state experimentally by selecting candidates within $100 \mathrm{MeV} / c^{2}$ of the mass [30,31]. In the LHCb experiment, however, no attempt is made to separate the vector $K^{* 0}$ from the $S$ wave or other broad contributions which may present in the selected $K^{+} \pi^{-}$pair [14]. Fortunately, the $S$-wave fraction contribution to the $B^{0} \rightarrow \bar{K}^{* 0} \mu^{+} \mu^{-}$mode has been measured by the LHCb and found to be small [13]. For the $B_{s}$ case, the $S$-wave contamination of the $B_{s}^{0} \rightarrow \bar{K}^{* 0} \mu^{+} \mu^{-}$ decay is also unknown now and assumed to be small compared to that of the $B^{0} \rightarrow \bar{K}^{* 0} \mu^{+} \mu^{-}$decay. Specifically, the $S$-wave fraction of $F_{S}\left(\bar{B}^{0} \rightarrow \bar{K}^{* 0} \mu^{+} \mu^{-}\right)=(3.4 \pm$ $0.8) \%$ in the $K^{+} \pi^{-}$system [20]. Theoretically, the authors of Ref. [32] found the $S$-wave contribution will modify differential decay widths by about $10 \%$ in the process of $\bar{B}^{0} \rightarrow K^{-} \pi^{+} \ell^{+} \ell^{-}$.

Analogous to the ratios $R_{K}$ and $R_{K^{*}}$ for $B \rightarrow K^{(*)} l^{+} l^{-}$ decays as defined in Refs. [1-14], we can define the similar ratios of the BRs $R_{s, K}^{e \mu}$ and $R_{s, K^{*}}^{e \mu}$ for the $\bar{B}_{s} \rightarrow K^{(*)} \ell^{+} \ell^{-}$ decays:

$$
R_{s, K^{(*)}}^{e \mu}=\frac{\mathcal{B}\left(\bar{B}_{s} \rightarrow K^{(*)} \mu^{+} \mu^{-}\right)}{\mathcal{B}\left(\bar{B}_{s} \rightarrow K^{(*)} e^{+} e^{-}\right)} .
$$

Similarly, we can also define the ratios $R_{s, K}^{\mu \tau}$ and $R_{s, K^{*}}^{\mu \tau}$ in the following form:

$$
R_{s, K^{(*)}}^{\mu \tau}=\frac{\mathcal{B}\left(\bar{B}_{s} \rightarrow K^{(*)} \tau^{+} \tau^{-}\right)}{\mathcal{B}\left(\bar{B}_{s} \rightarrow K^{(*)} \mu^{+} \mu^{-}\right)} .
$$

These new ratios $R_{s, K^{(*)}}^{e \mu}$ and $R_{s, K^{(*)}}^{\mu \tau}$, together with the ratios $R_{K}$ and $R_{K^{*}}$, can help us to examine the $b \rightarrow(s, d) \ell^{+} \ell^{-}$ transitions in great details.

Unlike the well-studied $B \rightarrow K^{(*)} \ell^{+} \ell^{-}$decays, the semileptonic $\bar{B}_{s} \rightarrow K^{(*)} \ell^{+} \ell^{-}$decays have not caught much attention partially due to their lower branching ratios and the lack of the relevant experimental measurements. In recent years, these decays have been studied by several authors, for example, in Refs. [26-29], and the first measured branching ratio as listed in Eq. (2) was reported last year by the LHCb Collaboration [20]. Besides the measurements for the branching ratios, a precise angular reconstruction of the polarized $K^{*}$ in $\bar{B}_{s} \rightarrow K^{(*)} \ell^{+} \ell^{-}$ decays was discussed in Ref. [8]. Recently, the predictions of several angular observables for the $\bar{B}_{s} \rightarrow K^{*} \ell^{+} \ell^{-}$ decays were provided using the light-cone sum rule (LCSR) and the lattice QCD method in Ref. [29].

By using the perturbative QCD (PQCD) factorization approach [33-35], the semileptonic $\bar{B}_{s} \rightarrow K \ell^{+} \ell^{+}$decays have been studied by us in a previous paper [26]. We considered the next-to-leading order (NLO) contributions known at 2012 and presented our PQCD predictions of the branching ratios:

$$
\begin{gathered}
\mathcal{B}\left(\bar{B}_{s}^{0} \rightarrow K^{0} \ell^{+} \ell^{-}\right)=\left(1.63_{-0.58}^{+0.73}\right) \times 10^{-8}, \quad l=(e, \mu), \\
\mathcal{B}\left(\bar{B}_{s}^{0} \rightarrow K^{0} \tau^{+} \tau^{-}\right)=\left(0.43_{-0.15}^{+0.18}\right) \times 10^{-8} .
\end{gathered}
$$

In this paper, we will make a systematic study for the semileptonic decays $\bar{B}_{s} \rightarrow\left(K, K^{*}\right) \ell^{+} \ell^{-}$with $l=(e, \mu, \tau)$, and present the theoretical predictions of many new physical observables:

(1) For $\bar{B}_{s} \rightarrow K \ell^{+} \ell^{-}$decays, besides the branching ratios, we also calculate their forward-backward (FB) asymmetry $\mathcal{A}_{F B}\left(q^{2}\right)$, the longitudinal lepton polarization asymmetry $P_{L}\left(q^{2}\right)$, the direct $C P$ asymmetry $\mathcal{A}_{C P}$, and the ratios $R_{s, K}^{e \mu}$ and $R_{s, K}^{\mu \tau}$.

(2) For $\bar{B}_{s} \rightarrow K^{*} \ell^{+} \ell^{-}$decays, we treat them as a four body decay $\bar{B}_{s} \rightarrow K^{*}(\rightarrow K \pi) \ell^{+} \ell^{-}$described by four kinematic variables: the lepton invariant mass squared $q^{2}$ and three angles $\left(\theta_{K^{*}}, \theta_{\ell}, \phi\right)$. We define and calculate the full angular decay distributions, the transverse amplitudes, the partially integrated decay amplitudes over the angles $\left(\theta_{K^{*}}, \theta_{\ell}, \phi\right)$, the FB asymmetry $\mathcal{A}_{F B}\left(q^{2}\right)$, the $K^{*}$ polarization fraction $R_{L, T}\left(q^{2}\right)$ and the longitudinal lepton polarization asymmetry $P_{L}\left(q^{2}\right)$, and the ratios $R_{s, K^{*}}^{e \mu}$ and $R_{s, K^{*}}^{\mu \tau}$. Since we do not know how to calculate the possible $S$-wave or other broad contributions related with the reconstruction of the $K \pi$ pair $[13,20]$, we add a $10 \%$ uncertainty to the PQCD predictions of the branching ratios as an additional theoretical error [32], but neglect it in the calculations for other ratios due to the strong cancellation.

(3) We use both the PQCD factorization approach and the "PQCD + Lattice" approach to determine the values and their $q^{2}$ dependence of the $\bar{B}_{s} \rightarrow K^{(*)}$ transition form factors. We use the BourrelyCaprini-Lellouch (BCL) parametrization method $[36,37]$ to make the extrapolation for all form factors from the low- $q^{2}$ region to $q_{\max }^{2}$. We will calculate the branching ratios and all other physical observables using the PQCD approach and the PQCD + Lattice 
approach, respectively, and compare the theoretical predictions obtained based on different models.

The paper is organized as follows: In Sec. II, we give a short review for the kinematics of the $\bar{B}_{s} \rightarrow K^{(*)} \ell^{+} \ell^{-}$ decays including the distribution amplitudes of $B_{s}$ and $K^{(*)}$ mesons. Section III is devoted to the theoretical framework including the Hamiltonian and transition form factors based on the PQCD $k_{T}$ factorization formalism. In Sec. IV, we list all the observables for both types of decays considered in this paper. Section V contains the numerical results of relevant observables and some phenomenological discussions. We conclude and summarize in the last section.

\section{KINEMATICS AND THE WAVE FUNCTIONS}

We discuss the kinematics of these decays in the largerecoil (low- $q^{2}$ ) region, where the PQCD factorization approach is applicable to the considered semileptonic decays involving $K^{(*)}$ as the final state meson. In the rest frame of $\bar{B}_{s}^{0}$ meson, we define the $\bar{B}_{s}^{0}$ meson momentum $p_{1}$, the $K^{(*)}$ momentum $p_{2}$ in the light-cone coordinates, as in Ref. [38]

$$
p_{1}=\frac{m_{B_{s}}}{\sqrt{2}}\left(1,1,0_{\perp}\right), \quad p_{2}=\frac{r m_{B_{s}}}{\sqrt{2}}\left(\eta^{+}, \eta^{-}, 0_{\perp}\right),
$$

where the mass ratio $r=m_{K} / m_{B_{s}}$ or $m_{K}^{*} / m_{B_{s}}$, and the factor $\eta^{ \pm}$is defined in the following form:

$\eta^{ \pm}=\eta \pm \sqrt{\eta^{2}-1}, \quad$ with $\quad \eta=\frac{1}{2 r}\left[1+r^{2}-\frac{q^{2}}{m_{B_{s}}^{2}}\right]$,

where $q=p_{1}-p_{2}$ is the lepton-pair four-momentum. For the final state $K^{*}$ meson, its longitudinal and transverse polarization vector $\epsilon_{L, T}$ can be written as

$$
\epsilon_{L}=\frac{1}{\sqrt{2}}\left(\eta^{+},-\eta^{-}, 0_{\perp}\right), \quad \epsilon_{T}=(0,0,1) .
$$

The momenta of the spectator quarks in $B_{s}$ and $K^{(*)}$ mesons are parametrized as

$k_{1}=\left(0, x_{1} \frac{m_{B_{s}}}{\sqrt{2}}, k_{1 \perp}\right), \quad k_{2}=\frac{m_{B_{s}}}{\sqrt{2}}\left(x_{2} r \eta^{+}, x_{2} r \eta^{-}, k_{2 \perp}\right)$.

We make the approximation in the small $k_{\perp}$.

For the $\bar{B}_{s}^{0}$ meson wave function, we use the same parametrizations as in Refs. [26,39]

$$
\Phi_{B_{s}}=\frac{i}{\sqrt{2 N_{c}}}\left(\not \not_{B_{s}}+m_{B_{s}}\right) \gamma_{5} \phi_{B_{s}}\left(k_{1}\right) .
$$

Here, only the contribution of the Lorentz structure $\phi_{B_{s}}\left(k_{1}\right)$ is taken into account, since the contribution of the second Lorentz structure $\bar{\phi}_{B_{s}}$ is numerically small and has been neglected. We adopted the $B_{s}$-meson distribution amplitude that was the same as the $B$ meson in the $S U(3)_{f}$ limit widely used in the PQCD approach

$\phi_{B_{s}}(x, b)=N_{B_{s}} x^{2}(1-x)^{2} \exp \left[-\frac{m_{B_{s}}^{2} x^{2}}{2 \omega_{B_{s}}^{2}}-\frac{1}{2}\left(\omega_{B_{s}} b\right)^{2}\right]$.

In order to analyze the uncertainties of theoretical predictions induced by the inputs, one usually takes $\omega_{B_{s}}=$ $0.50 \pm 0.05 \mathrm{GeV}$ for the $B_{s}^{0}$ meson. The normalization factor $N_{B_{s}}$ depends on the values of the shape parameter $\omega_{B_{s}}$ and the decay constant $f_{B_{s}}$ and is defined through the normalization relation: $\int_{0}^{1} d x \phi_{B_{s}}(x, b=0)=$ $f_{B_{s}} /(2 \sqrt{6})[26]$.

For the pseudoscalar $K$ meson, the wave function can be chosen as the same one in Ref. [40]:

$$
\begin{aligned}
\Phi_{K}(p, x) \equiv & \frac{i}{\sqrt{6}} \gamma_{5}\left[\not \phi_{K} \phi_{K}^{A}(x)+m_{0}^{K} \phi_{K}^{p}(x)\right. \\
& \left.+\zeta m_{0}^{K}(\not \not h-1) \phi_{K}^{T}(x)\right],
\end{aligned}
$$

where $m_{0}^{K}$ and $p$ are the chiral mass and the momentum of the meson $K$. The parameter $\zeta=1$ or -1 when the momentum fraction of the quark (antiquark) of the meson is set to be $x$. The distribution amplitudes (DAs) of the kaon meson can be found easily in Refs. [41-44]:

$$
\begin{aligned}
\phi_{K}^{A}(x)= & \frac{3 f_{K}}{\sqrt{6}} x(1-x)\left[1+a_{1}^{K} C_{1}^{3 / 2}(t)+a_{2}^{K} C_{2}^{3 / 2}(t)\right. \\
& \left.+a_{4}^{K} C_{4}^{3 / 2}(t)\right], \\
\phi_{K}^{P}(x)= & \frac{f_{K}}{2 \sqrt{6}}\left\{1+\left(30 \eta_{3}-\frac{5}{2} \rho_{K}^{2}\right) C_{2}^{1 / 2}(t)\right. \\
& \left.-3\left[\eta_{3} \omega_{3}+\frac{9}{20} \rho_{K}^{2}\left(1+6 a_{2}^{K}\right) C_{4}^{1 / 2}(t)\right]\right\}, \\
\phi_{K}^{T}(x)= & \frac{f_{K}(1-2 x)}{2 \sqrt{6}}\left\{1+6\left[5 \eta_{3}-\frac{1}{2} \eta_{3} \omega_{3}\right.\right. \\
& \left.\left.-\frac{7}{20} \rho_{K}^{2}-\frac{3}{5} \rho_{K}^{2} a_{2}^{K}\right]\left(1-10 x+10 x^{2}\right)\right\},
\end{aligned}
$$

where $t=2 x-1, f_{K}$ is the decay constant of the kaon meson and $\rho_{K}=m_{K} / m_{K}^{0}$ is the mass ratio. The Gegenbauer moments and other parameters are [41-44]

$a_{1}^{K}=0.06 \pm 0.03, \quad a_{2}^{K}=0.25 \pm 0.15$,

$a_{4}^{K}=-0.015, \quad \eta_{3}=0.015, \quad \omega=-3.0$.

The Gegenbauer polynomials that appeared in Eqs. (14) and (15) are of the following form [41-44]: 


$$
\begin{aligned}
& C_{1}^{3 / 2}(t)=3 t, \quad C_{2}^{1 / 2}(t)=\frac{1}{2}\left(3 t^{2}-1\right), \quad C_{2}^{3 / 2}(t)=\frac{3}{2}\left(5 t^{2}-1\right), \\
& C_{4}^{1 / 2}(t)=\frac{1}{8}\left(3-30 t^{2}+35 t^{4}\right), \quad C_{4}^{3 / 2}(t)=\frac{15}{8}\left(1-14 t^{2}+21 t^{4}\right) .
\end{aligned}
$$

For the light vector meson $K^{*}$, the longitudinal and transverse polarization components can provide the contribution. Here we adopt the wave functions of the vector $K^{*}$ as in Ref. [44]:

$$
\begin{gathered}
\Phi_{K^{*}}^{\|}\left(p, \epsilon_{L}\right)=\frac{i}{\sqrt{6}}\left[\phi_{L} m_{K^{*}} \phi_{K^{*}}(x)+\phi_{L} \not p \phi_{K^{*}}^{t}(x)+m_{K^{*}} \phi_{K^{*}}^{s}(x)\right], \\
\Phi_{K^{*}}^{\perp}\left(p, \epsilon_{T}\right)=\frac{i}{\sqrt{6}}\left[\phi_{T} m_{K^{*}} \phi_{K^{*}}^{v}(x)+\phi_{T} \not p \phi_{K^{*}}^{T}(x)+m_{K^{*}} i \epsilon_{\omega v \rho \sigma} \gamma_{5} \gamma^{\omega} \epsilon_{T}^{v} n^{\rho} v^{\sigma} \phi_{K^{*}}^{a}(x)\right],
\end{gathered}
$$

where $p$ and $m_{K^{*}}$ are the momentum and the mass of the $K^{*}$ meson, and $\epsilon_{L}$ and $\epsilon_{T}$ correspond to the longitudinal and transverse polarization vectors of the vector meson, respectively. The $\phi_{K^{*}}$ and $\phi_{K^{*}}^{T}$ in Eqs. (19) and (20) are the twist-two DAs [44]:

$$
\begin{aligned}
& \phi_{K^{*}}(x)=\frac{3 f_{K^{*}}}{\sqrt{6}} x(1-x)\left[1+a_{1 K^{*}}^{\|} C_{1}^{3 / 2}(t)+a_{2 K^{*}}^{\|} C_{2}^{3 / 2}(t)\right], \\
& \phi_{K^{*}}^{T}(x)=\frac{3 f_{K^{*}}^{T}}{\sqrt{6}} x(1-x)\left[1+a_{1 K^{*}}^{\perp} C_{1}^{3 / 2}(t)+a_{2 K^{*}}^{\perp} C_{2}^{3 / 2}(t)\right],
\end{aligned}
$$

where $f_{K^{*}}$ and $f_{K^{*}}^{T}$ are the longitudinal and transverse components of the decay constants. The Gegenbauer moments in Eqs. (19) and (20) are the same ones as those in Ref. [44]:

$$
\begin{array}{ll}
a_{1 K^{*}}^{\|}=0.03 \pm 0.02, & a_{2 K^{*}}^{\|}=0.11 \pm 0.09 \\
a_{1 K^{*}}^{\perp}=0.04 \pm 0.03, & a_{2 K^{*}}^{\perp}=0.10 \pm 0.08
\end{array}
$$

The twist-three DAs $\phi_{K^{*}}^{s, t}$ and $\phi_{K^{*}}^{v, a}$ in Eqs. (19) and (20) are defined with the asymptotic form as in Ref. [44]:

$$
\phi_{K^{*}}^{t}=\frac{3 f_{K^{*}}^{T}}{2 \sqrt{6}} t^{2}, \quad \phi_{K^{*}}^{s}=\frac{3 f_{K^{*}}^{T}}{2 \sqrt{6}}(-t), \quad \phi_{K^{*}}^{v}=\frac{3 f_{K^{*}}}{8 \sqrt{6}}\left(1+t^{2}\right), \quad \phi_{K^{*}}^{a}=\frac{3 f_{K^{*}}}{4 \sqrt{6}}(-t),
$$

\section{THEORETICAL FRAMEWORK}

\section{A. Effective Hamiltonian for $b \rightarrow d \ell^{+} \ell^{-}$decays}

For the considered $b \rightarrow d \ell^{+} \ell^{-}$transitions, the effective Hamiltonian in the framework of the SM can be written in the following form $[29,45-47]$ :

$$
\begin{aligned}
\mathcal{H}_{\mathrm{eff}}= & -\frac{G_{F}}{\sqrt{2}} V_{t b} V_{t d}^{*}\left\{C_{1}(\mu) \mathcal{O}_{1}^{c}(\mu)+C_{2}(\mu) \mathcal{O}_{2}^{c}(\mu)+\sum_{i=3}^{10} C_{i}(\mu) \mathcal{O}_{i}(\mu)\right. \\
& \left.+\lambda_{u}\left[C_{1}(\mu)\left(\mathcal{O}_{1}^{c}(\mu)-\mathcal{O}_{1}^{u}(\mu)\right)+C_{2}(\mu)\left(\mathcal{O}_{2}^{c}(\mu)-\mathcal{O}_{2}^{u}(\mu)\right)\right]\right\},
\end{aligned}
$$

where $G_{F}=1.16638 \times 10^{-5} \mathrm{GeV}^{-2}$ is the Fermi constant, $\lambda_{u}=V_{u b} V_{u d}^{*} /\left(V_{t b} V_{t d}^{*}\right)$ is a ratio of the CKM elements, and $C_{i}(\mu)$ and $\mathcal{O}_{i}(\mu)$ are the Wilson coefficients and the four-fermion operators at the renormalization scale $\mu$. In the SM, a suitable basis of the operators $\mathcal{O}_{i}(\mu)$ for the $b \rightarrow d \ell^{+} \ell^{-}$transition is given by the current-current operators $\mathcal{O}_{1,2}^{u, c}$, the QCD penguin operators $\mathcal{O}_{3-6}$, the electromagnetic penguin operator $\mathcal{O}_{7}$, and the chromomagnetic penguin operator $\mathcal{O}_{8}$, as well as the semileptonic operators $\mathcal{O}_{9,10}$ : 


$$
\begin{aligned}
\mathcal{O}_{1}^{c}=\left(\bar{d}_{\alpha} c_{\beta}\right)_{V-A}\left(\bar{c}_{\beta} b_{\alpha}\right)_{V-A}, & \mathcal{O}_{2}^{c}=\left(\bar{d}_{\alpha} c_{\alpha}\right)_{V-A}\left(\bar{c}_{\beta} b_{\beta}\right)_{V-A}, \\
\mathcal{O}_{1}^{u}=\left(\bar{d}_{\alpha} u_{\beta}\right)_{V-A}\left(\bar{u}_{\beta} b_{\alpha}\right)_{V-A}, & \mathcal{O}_{2}^{u}=\left(\bar{d}_{\alpha} u_{\alpha}\right)_{V-A}\left(\bar{u}_{\beta} b_{\beta}\right)_{V-A}, \\
\mathcal{O}_{3}=\left(\bar{d}_{\alpha} b_{\alpha}\right)_{V-A} \sum_{q}\left(\bar{q}_{\beta} q_{\beta}\right)_{V-A}, & \mathcal{O}_{4}=\left(\bar{d}_{\alpha} b_{\beta}\right)_{V-A} \sum_{q}\left(\bar{q}_{\beta} q_{\alpha}\right)_{V-A}, \\
\mathcal{O}_{5}=\left(\bar{d}_{\alpha} b_{\alpha}\right)_{V-A} \sum_{q}\left(\bar{q}_{\beta} q_{\beta}\right)_{V+A}, & \mathcal{O}_{6}=\left(\bar{d}_{\alpha} b_{\beta}\right)_{V-A} \sum_{q}\left(\bar{q}_{\beta} q_{\alpha}\right)_{V+A}, \\
\mathcal{O}_{7}=\frac{e m_{b}}{8 \pi^{2}} \bar{d} \sigma^{\mu \nu}\left(1+\gamma_{5}\right) b F_{\mu \nu}, & \mathcal{O}_{8}=\frac{g m_{b}}{8 \pi^{2}} \bar{d} \sigma^{\mu \nu} T^{a}\left(1+\gamma_{5}\right) b G_{\mu \nu}^{a}, \\
\mathcal{O}_{9}=\frac{\alpha_{\mathrm{em}}}{2 \pi}\left(\bar{d} \gamma^{\mu}\left(1-\gamma_{5}\right) b\right)\left(\bar{e}_{\mu} \ell\right), & \mathcal{O}_{10}=\frac{\alpha_{\mathrm{em}}}{2 \pi}\left(\bar{d} \gamma^{\mu}\left(1-\gamma_{5}\right) b\right)\left(\bar{\ell} \gamma_{\mu} \gamma_{5} \ell\right),
\end{aligned}
$$

where $T^{a}$ denotes the generators of the $S U(3)_{C}$ group and $m_{b}$ is the running $b$-quark mass in the $\overline{\mathrm{MS}}$ scheme; $F_{\mu \nu}$ and $G_{\mu \nu}^{a}$ are the electromagnetic and chromomagnetic tensors, respectively. The labels $V \pm A$ refer to the Lorentz structure $\gamma_{\mu}\left(1 \pm \gamma_{5}\right)$. In Fig. 1, we show the typical Feynman diagrams for the semileptonic decays $\bar{B}_{s}^{0} \rightarrow K^{(*)} l^{+} l^{-}$in the PQCD approach. The dominant contribution to the $b \rightarrow d \ell^{+} \ell^{-}$transitions is given by $\mathcal{O}_{7}$ and $\mathcal{O}_{9,10}$, as well as $\mathcal{O}_{1,2}^{u, c}$. The operator $\mathcal{O}_{7}$ corresponds to the $\gamma$-penguin diagram, as shown in Fig. 2(a). The operators $\mathcal{O}_{9,10}$ describe the sum of the contributions from the $Z$ and $\gamma$ penguin in Fig. 2(a) and the $W$ box diagrams in Fig. 2(b). The current-current operators $\mathcal{O}_{1,2}^{u, c}$ involve a long-distance contribution, which originates in the real $u \bar{u}, d \bar{d}$, and $c \bar{c}$ intermediate states, namely, the $(\rho, \omega, \phi)$ and $J / \psi$ family in Fig. 2(c), coupled to the lepton pair via the virtual photon. This contribution is proportional to $C_{9}$ and can be absorbed into an effective Wilson coefficient $C_{9}^{\text {eff }}$ [48].

Here we neglect the contribution from the subleading chromomagnetic penguin, and the quark-loop and annihilation diagrams because these effects are highly suppressed [29]. Hence, the decay amplitude for $b \rightarrow d l^{+} l^{-}$loop transition can be decomposed as

$$
\begin{aligned}
\mathcal{A}\left(b \rightarrow d l^{+} l^{-}\right)= & \frac{G_{F}}{2 \sqrt{2}} \frac{\alpha_{\mathrm{em}}}{\pi} V_{t b} V_{t d}^{*}\left\{C_{9}^{\mathrm{eff}}\left(q^{2}\right)\left[\bar{d} \gamma_{\mu}\left(1-\gamma_{5}\right) b\right]\left[\bar{l} \gamma^{\mu} l\right]+C_{10}\left[\bar{d} \gamma_{\mu}\left(1-\gamma_{5}\right) b\right]\left[\bar{l} \gamma^{\mu} \gamma_{5} l\right]\right. \\
& \left.-2 m_{b} C_{7}^{\mathrm{eff}}\left[\bar{d} i \sigma_{\mu \nu} \frac{q^{\nu}}{q^{2}}\left(1+\gamma_{5}\right) b\right]\left[\bar{l} \gamma^{\mu} l\right]\right\},
\end{aligned}
$$

where $C_{7}^{\mathrm{eff}}(\mu)$ and $C_{9}^{\mathrm{eff}}(\mu)$ are the effective Wilson coefficients, defined as in Refs. [26,49]

$$
\begin{gathered}
C_{7}^{\mathrm{eff}}(\mu)=C_{7}(\mu)+C_{b \rightarrow d \gamma}^{\prime}(\mu), \\
C_{9}^{\mathrm{eff}}\left(\mu, q^{2}\right)=C_{9}(\mu)+Y_{\text {pert }}(\hat{s})+Y_{\text {res }}\left(q^{2}\right) .
\end{gathered}
$$

The analytic expressions for all Wilson coefficients in the NLO approximation can be found easily in Ref. [50]. The numerical values of the NLO Wilson coefficients $C_{i}(\mu)$ at three different renormalization scales

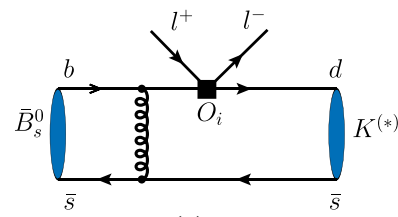

(a)

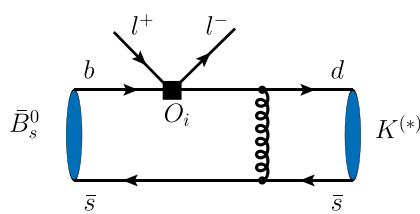

(b)
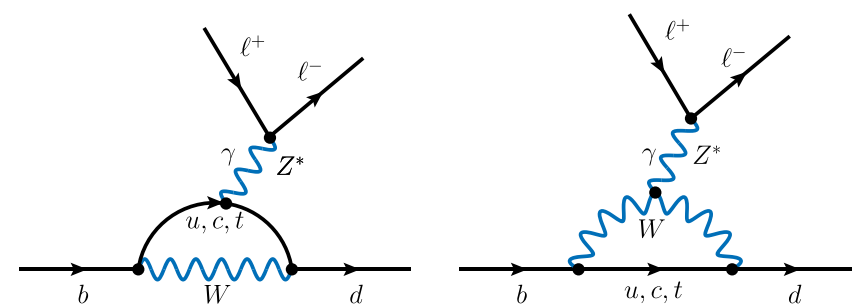

(a)

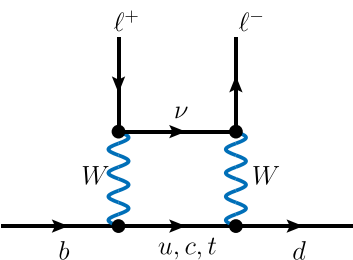

(b)

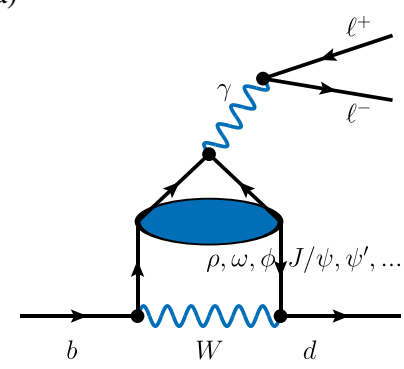

(c)
FIG. 1. The typical Feynman diagrams for the semileptonic decays $\bar{B}_{s}^{0} \rightarrow K^{(*)} \ell^{+} \ell^{-}$in the PQCD approach with the FCNC contributions due to the operators $O_{i}$ as defined in Eq. (26) and denoted as black squares in this figure.
FIG. 2. Typical Feynman loop diagrams: the $\gamma$ penguin (a) with $\mathcal{O}_{7}$, the $z(\gamma)$ penguin (a) and $W$ box (b) with $\mathcal{O}_{9,10}$, and the loops (c) with $\mathcal{O}_{1,2}^{u, c}$. 
TABLE I. The values of the Wilson coefficients $C_{i}(\mu)$ in NLO level at three different renormalization scales $\mu=\left(m_{b} / 2, m_{b}, 3 m_{b} / 2\right)$.

\begin{tabular}{lcccccccccr}
\hline \hline$\mu \backslash C_{i}(\mu)$ & $C_{1}$ & $C_{2}$ & $C_{3}(\%)$ & $C_{4}(\%)$ & $C_{5}(\%)$ & $C_{6}(\%)$ & $C_{7}$ & $C_{8}$ & $C_{9}$ & $C_{10}$ \\
\hline$m_{b} / 2$ & -0.276 & 1.131 & 2.005 & -4.845 & 1.375 & -5.841 & -0.329 & -0.165 & 4.450 & -4.410 \\
$m_{b}$ & -0.175 & 1.076 & 1.258 & -3.279 & 1.112 & -3.634 & -0.302 & -0.148 & 4.232 & -4.410 \\
$3 m_{b} / 2$ & -0.129 & 1.053 & 0.966 & -2.608 & 0.964 & -2.786 & -0.287 & -0.139 & 4.029 & -4.410 \\
\hline \hline
\end{tabular}

$\mu=\left(m_{b} / 2, m_{b}, 3 m_{b} / 2\right)$ are listed in Table I. Note that the Wilson coefficient $C_{10}$ is independent of the $\mu$ scale and $C_{9}(\mu)$ is relatively sensitive to the choice of $\mu$.

The term $C_{b \rightarrow d \gamma}^{\prime}$ in Eq. (28) is the absorptive part of $b \rightarrow d \gamma$ and was given in Ref. [49]

$$
C_{b \rightarrow d \gamma}^{\prime}(\mu)=i \alpha_{s}\left\{\frac{2}{9} \eta^{14 / 23}\left[G_{I}\left(x_{t}\right)-0.1687\right]-0.03 C_{2}(\mu)\right\}
$$

where $\eta=\alpha_{s}\left(m_{W}\right) / \alpha_{s}(\mu), x_{t}=m_{t}^{2} / m_{W}^{2}$ and

$$
G_{I}\left(x_{t}\right)=\frac{x_{t}\left(x_{t}^{2}-5 x_{t}-2\right)}{8\left(x_{t}-1\right)^{3}}+\frac{3 x_{t}^{2} \ln x_{t}}{4\left(x_{t}-1\right)^{4}}
$$

Besides the ordinary Wilson coefficient $C_{9}(\mu)$, the effective Wilson coefficient $C_{9}^{\text {eff }}\left(q^{2}\right)$ in Eq. (29) also contains two additional effective terms $Y_{\text {pert }}(\hat{s})$ and $Y_{\text {res }}\left(q^{2}\right)$. The term $Y_{\text {pert }}(\hat{s})$ describes the short distance contribution from the soft-gluon emission and the one-loop contribution of the four-quark operators $\mathcal{O}_{1}-\mathcal{O}_{6}$. The term $Y_{\text {res }}\left(q^{2}\right)$ includes the contributions of the virtual resonances described by the Breit-Wigner form prescribed in Refs. [47,51-54].

$$
\begin{aligned}
Y_{\text {pert }}(\hat{s})= & 0.124 \omega(\hat{s})+g\left(\hat{m}_{c}, \hat{s}\right) C_{0}+\lambda_{u}\left[g\left(\hat{m}_{c}, \hat{s}\right)-g\left(\hat{m}_{u}, \hat{s}\right)\right]\left(3 C_{1}+C_{2}\right) \\
& -\frac{1}{2} g\left(\hat{m}_{d}, \hat{s}\right)\left(C_{3}+3 C_{4}\right)-\frac{1}{2} g\left(\hat{m}_{b}, \hat{s}\right)\left(4 C_{3}+4 C_{4}+3 C_{5}+C_{6}\right) \\
+ & \frac{2}{9}\left(3 C_{3}+C_{4}+3 C_{5}+C_{6}\right), \\
Y_{\text {res }}\left(q^{2}\right)= & -\frac{3 \pi}{\alpha_{\mathrm{em}}^{2}}\left[C_{0} \cdot \sum_{V=J / \Psi, \Psi^{\prime} \ldots} \frac{m_{V} \mathcal{B}\left(V \rightarrow l^{+} l^{-}\right) \Gamma_{\text {tot }}^{V}}{q^{2}-m_{V}^{2}+i m_{V} \Gamma_{\text {tot }}^{V}}\right. \\
& \left.\quad-\lambda_{u} g\left(\hat{m}_{u}, \hat{s}\right)\left(3 C_{1}+C_{2}\right) \cdot \sum_{V=\rho, \omega, \phi} \frac{m_{V} \mathcal{B}\left(V \rightarrow l^{+} l^{-}\right) \Gamma_{\text {tot }}^{V}-m_{V}^{2}+i m_{V} \Gamma_{\text {tot }}^{V}}{q^{2}}\right],
\end{aligned}
$$

where $C_{0}=3 C_{1}+C_{2}+3 C_{3}+C_{4}+3 C_{5}+C_{6}, \hat{s}=q^{2} / m_{b}^{2}$, and $\hat{m}_{q}=m_{q} / m_{b}$. In the above expressions, $\omega(\hat{s})$ is the softgluon correction to the matrix element of operator $\mathcal{O}_{9}$ and was given in Refs. [47,55]

$$
\begin{aligned}
\omega(\hat{s})= & -\frac{2}{9} \pi^{2}+\frac{4}{3} \int_{0}^{\hat{s}} \frac{\ln (1-u)}{u} d u-\frac{2}{3} \ln (\hat{s}) \ln (1-\hat{s})-\frac{5+4 \hat{s}}{3(1+2 \hat{s})} \ln (1-\hat{s}) \\
& -\frac{2 \hat{s}(1+\hat{s})(1-2 \hat{s})}{3(1-\hat{s})^{2}(1+2 \hat{s})} \ln \hat{s}+\frac{5+9 \hat{s}-6 \hat{s}^{2}}{6(1-\hat{s})(1+2 \hat{s})} .
\end{aligned}
$$

The loop coefficient functions $g\left(\hat{m}_{q}, \hat{s}\right)$ in Eqs. (32) and (33) describe the one-loop $(q \bar{q})$ contributions to the four-quark operators $\mathcal{O}_{1}-\mathcal{O}_{6}$, and can be written as the well-known expression [56-59]:

$$
g\left(\hat{m}_{q}, \hat{s}\right)=-\frac{8}{9} \ln \left(\hat{m}_{q}\right)+\frac{8}{27}+\frac{4}{9} x-\frac{2}{9}(2+x) \sqrt{|1-x|} \times \begin{cases}2 \arctan \frac{1}{\sqrt{x-1}} & , x>1 \\ \ln \left|\frac{1+\sqrt{1-x}}{1-\sqrt{1-x}}\right|-i \pi & , x<1,\end{cases}
$$

where $x \equiv 4 \hat{m}_{q}^{2} / \hat{s}$.

In Ref. [59], the authors employed the dispersion approach to compute the charm-loop effect in a form of the correction to the Wilson coefficient $C_{9}$. By fitting the whole dispersion relation to the operator-product expansion
(OPE) result at $q^{2} \ll 4 m_{c}^{2}$, the authors found that there exists a destructive interference between the $J / \psi$ and $\psi(2 S)$ states. According to their opinion, a reliable prediction for the charm-loop effect above $\psi(2 S)$ based on QCD is hard to make. Although the actual effect, 
TABLE II. The masses, decay widths and branching fractions of the dilepton decays of the vector charmonium states [30].

\begin{tabular}{lccc}
\hline \hline & $\begin{array}{c}\text { Mass } \\
{[\mathrm{GeV}]}\end{array}$ & $\begin{array}{c}\Gamma_{\text {tot }}^{V} \\
{[\mathrm{MeV}]}\end{array}$ & $\begin{array}{c}\mathcal{B R}\left(V \rightarrow l^{+} l^{-}\right) \\
\text {with } l=e, \mu\end{array}$ \\
\hline$\rho(770)$ & 0.775 & 149.1 & $4.63 \times 10^{-5}$ \\
$\omega(782)$ & 0.782 & 8.490 & $7.38 \times 10^{-5}$ \\
$\phi(1020)$ & 1.019 & 4.249 & $2.92 \times 10^{-4}$ \\
$J / \psi(1 S)$ & 3.096 & 0.093 & $5.96 \times 10^{-2}$ \\
$\psi(2 S)$ & 3.686 & 0.294 & $7.96 \times 10^{-3}$ \\
$\psi(3770)$ & 3.773 & 27.2 & $9.60 \times 10^{-6}$ \\
$\psi(4040)$ & 4.039 & 80 & $1.07 \times 10^{-5}$ \\
$\psi(4160)$ & 4.191 & 70 & $6.90 \times 10^{-6}$ \\
$\psi(4415)$ & 4.421 & 62 & $9.40 \times 10^{-6}$ \\
\hline \hline
\end{tabular}

depending on the interference of many charmonium states, cannot be reliably constrained by OPE, it could be considered as small in this region.

The term $Y_{\text {res }}$ in Eq. (33) denotes the long-distance resonance contributions from those $B_{s} \rightarrow K^{(*)} V \rightarrow$ $K^{(*)}\left(V \rightarrow \ell^{+} \ell^{-}\right)$transitions, where $V$ stands for the possible intermediate resonance states decaying to lepton pairs:

(1) The charmless light vector mesons $V=(\rho, \omega, \phi)$. The kinematic region where the light resonances $(\rho, \omega, \phi)$ contribute is typically not excluded from the experimental analyses because their effects on branching fractions and other physical observables might be substantial [60].

(2) The $c \bar{c}$ charmonia $V_{c \bar{c}}=\psi(1 S, 2 S, 3770,4040$, $4160,4415)$. The two lowest charmonium states $\psi(1 S)$ and $\psi(2 S)$ (i.e., $J / \psi$ and $\psi^{\prime}$ ), whose masses are below the open charm threshold $(D \bar{D})$, have tiny width and can induce large breaking of quarkhadron duality. Hence, the narrow charmonia resonance regions are routinely rejected in the theoretical and experimental analysis. For the four higher charmonium resonances, however, they are broad and overlapping throughout the high- $q^{2}$ regions. One usually makes the integration over the full high- $q^{2}$ range.
As reported in Ref. [61], a resonance above $\psi(2 S)$ compatible with the $\psi(4160)$ has been observed by LHCb in the $B \rightarrow K \mu^{+} \mu^{-}$decay. Consequently, nearly all available contributions about the $J^{P C}=1^{--}$charmonium resonances above the open charm threshold should be taken into account [62]. In Table II, we list the properties of all considered intermediate resonance states: their mass, width, and branching fractions of the leptonic decay channel $V \rightarrow$ $l^{+} l^{-}$[30]. For the case $\ell=\tau$, only the fraction of $J / \psi(2 S) \rightarrow \tau^{+} \tau^{-}$does not vanish, which equals $3.1 \times$ $10^{-3}$ from Ref. [30].

\section{B. $B_{s} \rightarrow K, K^{*}$ transition form factors}

The $B_{s} \rightarrow K$ transition can be induced by the vector current $V^{\mu}$ and the tensor currents $T^{\mu \nu}$ :

$$
\left\langle K\left(p_{2}\right)\left|V^{\mu}\right| B_{s}\left(p_{1}\right)\right\rangle=f_{1}\left(q^{2}\right) p_{1}^{\mu}+f_{2}\left(q^{2}\right) p_{2}^{\mu},
$$

$\left\langle K\left(p_{2}\right)\left|T^{\mu \nu}\right| B_{s}\left(p_{1}\right)\right\rangle=i \frac{2}{m_{B_{s}}+m_{K}}\left[p_{2}^{\mu} q^{\nu}-q^{\mu} p_{2}^{\nu}\right] F_{T}\left(q^{2}\right)$,

where $V^{\mu}=\bar{d} \gamma^{\mu} b$ and $T^{\mu \nu}=\bar{d} \sigma^{\mu \nu} b$, and $q=p_{1}-p_{2}$ is the momentum carried off by the lepton pairs and $\sigma^{\mu \nu}=i\left[\gamma^{\mu}, \gamma^{\nu}\right] / 2$.

The $B_{s} \rightarrow K$ transition form factors $F_{+}\left(q^{2}\right)$ and $F_{0}\left(q^{2}\right)$ can be written as a combination of the auxiliary form factors $f_{1}\left(q^{2}\right)$ and $f_{2}\left(q^{2}\right)$ in Eq. (36):

$$
F_{+}\left(q^{2}\right)=\frac{1}{2}\left[f_{1}\left(q^{2}\right)+f_{2}\left(q^{2}\right)\right]
$$

$F_{0}\left(q^{2}\right)=F_{+}\left(q^{2}\right)+\frac{1}{2}\left[f_{1}\left(q^{2}\right)-f_{2}\left(q^{2}\right)\right] \frac{q^{2}}{m_{B_{s}}^{2}-m_{K}^{2}}$.

We also have the relation $F_{+}(0)=F_{0}(0)$ in order to smear the pole at $q^{2}=0$.

Using the well-studied wave functions as given in Sec. II, we calculated the three $B_{s} \rightarrow K$ form factors $f_{1}\left(q^{2}\right)$, $f_{2}\left(q^{2}\right)$, and $F_{T}\left(q^{2}\right)$ in the PQCD factorization approach:

$$
\begin{aligned}
f_{1}\left(q^{2}\right)= & 16 \pi m_{B_{s}}^{2} C_{F} \int d x_{1} d x_{2} \int b_{1} d b_{1} b_{2} d b_{2} \phi_{B_{s}}\left(x_{1}\right) \\
& \times\left\{\left[-x_{2} r^{2} \phi_{K}^{A}\left(x_{2}\right)+r_{0} \phi_{K}^{P}\left(x_{2}\right)-\frac{\eta+2 x_{2} r}{\sqrt{\eta^{2}-1}} r_{0} \phi_{K}^{T}\left(x_{2}\right)\right] \cdot H_{1}\left(t_{1}\right)\right. \\
& +\left[\left(x_{1}\left(\eta+\sqrt{\eta^{2}-1}\right)-r+\frac{x_{1}}{2 \sqrt{\eta^{2}-1}}\right) r \phi_{K}^{A}\left(x_{2}\right)\right. \\
& \left.\left.-x_{1}\left(1+\frac{\eta}{\sqrt{\eta^{2}-1}}\right) r_{0} \phi_{K}^{P}\left(x_{2}\right)\right] \cdot H_{2}\left(t_{2}\right)\right\},
\end{aligned}
$$




$$
\begin{aligned}
f_{2}\left(q^{2}\right)= & 16 \pi m_{B_{s}}^{2} C_{F} \int d x_{1} d x_{2} \int b_{1} d b_{1} b_{2} d b_{2} \phi_{B_{s}}\left(x_{1}\right) \\
& \times\left\{\left[\left(1+x_{2} r \eta\right) \phi_{K}^{A}\left(x_{2}\right)-2 x_{2} r_{0} \phi_{K}^{P}\left(x_{2}\right)-\frac{1-2 x_{2} r \eta}{r \sqrt{\eta^{2}-1}} r_{0} \phi_{K}^{T}\left(x_{2}\right)\right] \cdot H_{1}\left(t_{1}\right)\right. \\
& \left.+\left[-\frac{x_{1}}{2}\left(1+\frac{\eta}{\sqrt{\eta^{2}-1}}\right) \phi_{K}^{A}\left(x_{2}\right)+\left(2+\frac{x_{1}}{r \sqrt{\eta^{2}-1}}\right) r_{0} \phi_{K}^{P}\left(x_{2}\right)\right] \cdot H_{2}\left(t_{2}\right)\right\}, \\
F_{T}\left(q^{2}\right)= & 8 \pi m_{B_{s}}^{2} C_{F}(1+r) \int d x_{1} d x_{2} \int b_{1} d b_{1} b_{2} d b_{2} \phi_{B_{s}}\left(x_{1}\right) \\
& \times\left\{\left[\phi_{K}^{A}\left(x_{2}\right)-x_{2} r_{0} \phi_{K}^{P}\left(x_{2}\right)-\frac{1+x_{2} r \eta}{r \sqrt{\eta^{2}-1}} r_{0} \phi_{K}^{T}\left(x_{2}\right)\right] \cdot H_{1}\left(t_{1}\right)\right. \\
& \left.+\left[-\frac{x_{1}}{2}\left(1+\frac{\eta}{\sqrt{\eta^{2}-1}}\right) \phi_{K}^{A}\left(x_{2}\right)+\left(2+\frac{x_{1}}{r \sqrt{\eta^{2}-1}}\right) r_{0} \phi_{K}^{P}\left(x_{2}\right)\right] \cdot H_{2}\left(t_{2}\right)\right\} .
\end{aligned}
$$

where $C_{F}=4 / 3$ is a color factor, $r_{0}=m_{K}^{0} / m_{B_{s}}, r=m_{k} / m_{B_{s}}, \eta$ is defined in Eq. (8), and the function $H_{i}\left(t_{i}\right)$ in the following form:

$$
H_{i}\left(t_{i}\right)=h_{i}\left(x_{1}, x_{2}, b_{1}, b_{2}\right) \cdot \alpha_{s}\left(t_{i}\right) \exp \left[-S_{a b}\left(t_{i}\right)\right], \quad \text { for } i=(1,2) .
$$

The explicit expressions of the hard functions $h_{1,2}\left(x_{1}, x_{2}, b_{1}, b_{2}\right)$, the hard scales $t_{1,2}$, and the Sudakov factors $S_{a b}\left(t_{i}\right)$ will be given in the Appendix.

For the vector meson $K^{*}$ with polarization vector $\epsilon^{*}$, the relevant form factors for $B_{s} \rightarrow K^{*}$ transitions are $V\left(q^{2}\right)$ and $A_{0,1,2}\left(q^{2}\right)$ of the vector and axial-vector currents, and $T_{1,2,3}\left(q^{2}\right)$ of the tensor currents. In the PQCD factorization approach, these seven form factors of $B_{s} \rightarrow K^{*} \ell^{+} \ell^{-}$decays can be calculated and written in the following form:

$$
\begin{aligned}
V\left(q^{2}\right)= & 8 \pi m_{B_{s}}^{2} C_{F}(1+r) \int d x_{1} d x_{2} \int b_{1} d b_{1} b_{2} d b_{2} \phi_{B_{s}}\left(x_{1}\right) \\
& \times\left\{\left[-x_{2} r \phi_{K^{*}}^{v}\left(x_{2}\right)+\phi_{K^{*}}^{T}\left(x_{2}\right)+\frac{1+x_{2} r \eta}{\sqrt{\eta^{2}-1}} \phi_{K^{*}}^{a}\left(x_{2}\right)\right] \cdot H_{1}\left(t_{1}\right)\right. \\
& \left.+\left[\left(r+\frac{x_{1}}{2 \sqrt{\eta^{2}-1}}\right) \phi_{K^{*}}^{v}\left(x_{2}\right)-\frac{x_{1}-2 r \eta}{2 \sqrt{\eta^{2}-1}} \phi_{K^{*}}^{a}\left(x_{2}\right)\right] \cdot H_{2}\left(t_{2}\right)\right\} \\
A_{0}\left(q^{2}\right)= & 8 \pi m_{B_{s}}^{2} C_{F} \int d x_{1} d x_{2} \int b_{1} d b_{1} b_{2} d b_{2} \phi_{B_{s}}\left(x_{1}\right) \times\left\{\left[\left(1+x_{2} r(2 \eta-r)\right) \phi_{K^{*}}\left(x_{2}\right)\right.\right. \\
+ & \left.\left(1-2 x_{2}\right) r \phi_{K^{*}}^{t}\left(x_{2}\right)+\frac{(1-r \eta)-2 x_{2} r(\eta-r)}{\sqrt{\eta^{2}-1}} \phi_{K^{*}}^{s}\left(x_{2}\right)\right] \cdot H_{1}\left(t_{1}\right) \\
+ & {\left[\left[\frac{x_{1}}{\sqrt{\eta^{2}-1}}\left(\frac{\eta+r}{2}-r \eta^{2}\right)+\left(\frac{x_{1}}{2}-x_{1} r \eta+r^{2}\right)\right] \phi_{K^{*}}\left(x_{2}\right)\right.} \\
& -\left[\frac{x_{1}(1-r \eta)+2 r(r-\eta)}{\left.\left.\left.\sqrt{\eta^{2}-1}-x_{1} r\right] \phi_{K^{*}}^{s}\left(x_{2}\right)\right] \cdot H_{2}\left(t_{2}\right)\right\}}\right. \\
A_{1}\left(q^{2}\right)= & 16 \pi m_{B_{s}}^{2} C_{F} \frac{r}{1+r} \int d x_{1} d x_{2} \int b_{1} d b_{1} b_{2} d b_{2} \phi_{B_{s}}\left(x_{1}\right) \\
\times & \left\{\left[\left(1+x_{2} r \eta\right) \phi_{K^{*}}^{v}\left(x_{2}\right)+\left(\eta-2 x_{2} r\right) \phi_{K^{*}}^{T}\left(x_{2}\right)+x_{2} r \sqrt{\eta^{2}-1} \phi_{K^{*}}^{a}\left(x_{2}\right)\right] \cdot H_{1}\left(t_{1}\right)\right. \\
+ & {\left.\left[\left(r \eta-\frac{x_{1}}{2}\right) \phi_{K^{*}}^{v}\left(x_{2}\right)+\left(r \sqrt{\eta^{2}-1}+\frac{x_{1}}{2}\right) \phi_{K^{*}}^{a}\left(x_{2}\right)\right] \cdot H_{2}\left(t_{2}\right)\right\}, }
\end{aligned}
$$




$$
\begin{aligned}
& A_{2}\left(q^{2}\right)=\frac{(1+r)^{2}(\eta-r)}{2 r\left(\eta^{2}-1\right)} A_{1}\left(q^{2}\right)-8 \pi m_{B_{s}}^{2} C_{F} \frac{1+r}{\eta^{2}-r} \int d x_{1} d x_{2} \int b_{1} d b_{1} b_{2} d b_{2} \phi_{B_{s}}\left(x_{1}\right) \\
& \times\left\{\left[\left[\eta\left(1-x_{2} r^{2}\right)+r\left(x_{2}\left(2 \eta^{2}-1\right)-1\right)\right] \phi_{K^{*}}\left(x_{2}\right)+\left[1+2 x_{2} r^{2}-\left(1+2 x_{2}\right) r \eta\right] \phi_{K^{*}}^{t}\left(x_{2}\right)\right.\right. \\
& \left.+r\left(1-2 x_{2}\right) \sqrt{\eta^{2}-1} \phi_{K^{*}}^{s}\left(x_{2}\right)\right] \cdot H_{1}\left(t_{1}\right) \\
& +\left[\left[\left(r \eta-\frac{1}{2}\right) x_{1} \sqrt{\eta^{2}-1}-\left[r\left(r \eta-1-x_{1} \eta^{2}\right)+\frac{x_{1}(r+\eta)}{2}\right]\right] \phi_{K^{*}}\left(x_{2}\right)\right. \\
& \left.\left.+\left[x_{1}(r \eta-1)+\left(x_{2}-2\right) r \sqrt{\eta^{2}-1}\right] \phi_{K^{*}}^{s}\left(x_{2}\right)\right] \cdot H_{2}\left(t_{2}\right)\right\} \\
& T_{1}\left(q^{2}\right)=8 \pi m_{B_{s}}^{2} C_{F} \int d x_{1} d x_{2} \int b_{1} d b_{1} b_{2} d b_{2} \phi_{B_{s}}\left(x_{1}\right) \times\left\{\left[\left(1-2 x_{2}\right) r \phi_{K^{*}}^{v}\left(x_{2}\right)\right.\right. \\
& \left.+\left(1+2 x_{2} r \eta-x_{2} r^{2}\right) \phi_{K^{*}}^{T}\left(x_{2}\right)+\frac{1+2 x_{2} r^{2}-\left(1+2 x_{2}\right) r \eta}{\sqrt{\eta^{2}-1}} \phi_{K^{*}}^{a}\left(x_{2}\right)\right] \cdot H_{1}\left(t_{1}\right) \\
& \left.+\left[\left[\left(1-\frac{x_{1}}{2}\right) r-\frac{x_{1}(r \eta-1)}{2 \sqrt{\eta^{2}-1}}\right] \phi_{K^{*}}^{v}\left(x_{2}\right)+\left[\frac{r(\eta-r)}{\sqrt{\eta^{2}-1}}+\frac{x_{1}}{2}\left(r+\frac{r \eta-1}{\sqrt{\eta^{2}-1}}\right)\right] \phi_{K^{*}}^{a}\left(x_{2}\right)\right] \cdot H_{2}\left(t_{2}\right)\right\}, \\
& T_{2}\left(q^{2}\right)=16 \pi m_{B_{s}}^{2} C_{F} \frac{r}{1-r^{2}} \int d x_{1} d x_{2} \int b_{1} d b_{1} b_{2} d b_{2} \phi_{B_{s}}\left(x_{1}\right) \\
& \times\left\{\left[\left(1-\left(1+2 x_{2}\right) r \eta+2 x_{2} r^{2}\right) \phi_{K^{*}}^{v}\left(x_{2}\right)\right.\right. \\
& \left.+\left[x_{2} r \eta(2 \eta-r)-x_{2} r+\eta-r\right] \phi_{K^{*}}^{T}\left(x_{2}\right)+\left(1-2 x_{2}\right) r \sqrt{\eta^{2}-1} \phi_{K^{*}}^{a}\left(x_{2}\right)\right] \cdot H_{1}\left(t_{1}\right) \\
& +\left[\left[\frac{x_{2}}{2}\left(1+\frac{\eta}{\sqrt{\eta^{2}-1}}\right)(r \eta-1)+\left(r+\frac{x_{1}}{2 \sqrt{\eta^{2}-1}}\right)(\eta-r)\right] \phi_{K^{*}}^{v}\left(x_{2}\right)\right. \\
& \left.\left.+\left[\left(1-\frac{x_{1}}{2}\right) r \sqrt{\eta^{2}-1}+\frac{x_{1}}{2}(1-r \eta)\right] \phi_{K^{*}}^{a}\left(x_{2}\right)\right] \cdot H_{2}\left(t_{2}\right)\right\} \text {, } \\
& T_{3}\left(q^{2}\right)=\frac{(1-r)^{2}(\eta+r)}{2 r\left(\eta^{2}-1\right)} T_{2}\left(q^{2}\right)-8 \pi m_{B_{s}}^{2} C_{F} \frac{1-r^{2}}{\eta^{2}-1} \int d x_{1} d x_{2} \int b_{1} d b_{1} b_{2} d b_{2} \phi_{B_{s}}\left(x_{1}\right) \\
& \times\left\{\left[\frac{\eta^{2}-\left(1+2 x_{2}\right) r \eta+2 x_{2} r^{2}}{\eta-r} \phi_{K^{*}}\left(x_{2}\right)+\left(1+x_{2} r \eta\right) \phi_{K^{*}}^{t}\left(x_{2}\right)+x_{2} r \sqrt{\eta^{2}-1} \phi_{K^{*}}^{s}\left(x_{2}\right)\right] \cdot H_{1}\left(t_{1}\right)\right. \\
& \left.+\left[\left[r-\frac{x_{1}}{2}\left(\eta+\sqrt{\eta^{2}-1}\right)\right] \phi_{K^{*}}\left(x_{2}\right)+\left(x_{1}+2 r \sqrt{\eta^{2}-1}\right) \phi_{K^{*}}^{s}\left(x_{2}\right)\right] \cdot H_{2}\left(t_{2}\right)\right\} \text {, }
\end{aligned}
$$

where $r=m_{K^{*}} / m_{B_{s}}$, the twist-two DAs $\left(\phi_{K^{*}}, \phi_{K^{*}}^{T}\right)$ and other four twist-three DAs are defined in Eqs. (19), (20), and (24), and the functions $H_{1,2}\left(t_{1,2}\right)$ are the same ones as those defined in Eq. (43) for $B_{s} \rightarrow K$ transition, but with a replacement of $r=m_{K} / m_{B_{s}}$ by $r=m_{K^{*}} / m_{B_{s}}$.

\section{OBSERVABLES FOR $B_{s} \rightarrow K^{(*)} \ell^{+} \ell^{-}$DECAYS}

\section{A. Observables for $\boldsymbol{B}_{s} \rightarrow K \boldsymbol{\ell}^{+} \boldsymbol{\ell}^{-}$decays}

Within the SM operator basis, the decay amplitude of $B_{s} \rightarrow K \ell^{+} \ell^{-}$decay can be written in the following form [63]:

$$
\mathcal{A}\left(\bar{B}_{s}^{0} \rightarrow K \ell^{+} \ell^{-}\right)=\frac{G_{\mathrm{F}} \alpha_{e m}}{\sqrt{2} \pi} V_{t b} V_{t d}^{*}\left[\delta_{V} p_{1}^{\mu}\left(\bar{\ell} \gamma_{\mu} \ell\right)+\delta_{A} p_{1}^{\mu}\left(\bar{\ell} \gamma_{\mu} \gamma_{5} \ell\right)+\delta_{P}\left(\bar{\ell} \gamma_{5} \ell\right)\right]
$$


with

$$
\begin{aligned}
& \delta_{A}=C_{10} F_{+}\left(q^{2}\right), \\
& \delta_{V}=C_{9}^{\mathrm{eff}} F_{+}\left(q^{2}\right)+C_{7}^{\mathrm{eff}} \frac{2\left(m_{b}-m_{d}\right)}{m_{B_{s}}+m_{K}} F_{T}\left(q^{2}\right), \\
& \delta_{P}=-m_{\ell} C_{10}\left\{F_{+}\left(q^{2}\right)+\frac{m_{B_{s}}^{2}-m_{K}^{2}}{q^{2}}\left[F_{+}\left(q^{2}\right)-F_{0}\left(q^{2}\right)\right]\right\},
\end{aligned}
$$

where $p_{1}^{\mu}$ denotes the four-momentum of the $B_{s}$ meson and $m_{\ell}$ is the lepton mass.

Based on the matrix elements of the operators in terms of the form factors, we obtain the double differential decay rate for $\bar{B}_{s} \rightarrow K \ell^{+} \ell^{-}$with respect to $q^{2}$ and $\theta_{\ell}$ with lepton flavor $\ell$ [64],

$$
\frac{d^{2} \Gamma}{d q^{2} d \cos \theta_{\ell}}=a_{\ell}\left(q^{2}\right)+b_{\ell}\left(q^{2}\right) \cos \theta_{\ell}+c_{\ell}\left(q^{2}\right) \cos ^{2} \theta_{\ell} .
$$

The angle $\theta_{\ell}$ is defined as the angle between the $\bar{B}_{s}$ direction and the $\ell^{-}$direction in the $\ell^{+} \ell^{-}$rest frame. The corresponding angular coefficients $a_{\ell}, b_{\ell}$, and $c_{\ell}$ can be written as $[63,64]$

$$
\begin{gathered}
a_{\ell}\left(q^{2}\right)=\mathcal{N}\left[q^{2}\left|\delta_{P}\right|^{2}+\frac{\lambda}{4}\left(\left|\delta_{A}\right|^{2}+\left|\delta_{V}\right|^{2}\right)+4 m_{\ell}^{2} m_{B_{s}}^{2}\left|\delta_{A}\right|^{2}\right. \\
\left.+2 m_{\ell}\left(m_{B_{s}}^{2}-m_{K}^{2}+q^{2}\right) \operatorname{Re}\left(\delta_{P} \delta_{A}^{*}\right)\right] \\
b_{\ell}\left(q^{2}\right)=0 \\
c_{\ell}\left(q^{2}\right)=-\frac{\mathcal{N} \lambda \beta_{\ell}^{2}}{4}\left(\left|\delta_{A}\right|^{2}+\left|\delta_{V}\right|^{2}\right)
\end{gathered}
$$

with the factor $\mathcal{N}$,

$$
\mathcal{N}=\frac{G_{F}^{2} \alpha_{e m}^{2}\left|V_{t b} V_{t d}^{*}\right|^{2}}{2^{9} \pi^{5} m_{B_{s}}^{3}} \beta_{\ell} \sqrt{\lambda}
$$

where $\beta_{l}=\sqrt{1-4 \hat{m}_{l}^{2}}$ with $\hat{m}_{l}=m_{l} / \sqrt{q^{2}}, \alpha_{e m}=1 / 137$ is the fine structure constant, $m_{\ell}$ means the lepton mass, and $\lambda=\lambda\left(m_{B_{s}}^{2}, m_{K}^{2}, q^{2}\right)$ is the Källen function: $\lambda(a, b, c)=$ $a^{2}+b^{2}+c^{2}-2(a b+b c+c a)$.

Integration over the polar angle $\theta_{\ell}$ leads to the expression for the differential decay rate,

$$
\frac{d \Gamma}{d q^{2}}=2 a_{\ell}\left(q^{2}\right)+\frac{2}{3} c_{\ell}\left(q^{2}\right) .
$$

We see that the linear dependence on $\cos \theta_{\ell}$ is lost after integration over $\theta_{\ell}$, consequently, the lepton forwardbackward asymmetry $\mathcal{A}_{\mathrm{FB}}$ will also become zero,

$$
\begin{aligned}
\mathcal{A}_{\mathrm{FB}}\left(q^{2}\right) & =\frac{\int_{0}^{1} \frac{d^{2} \Gamma}{d q^{2} d \cos \theta_{\ell}} d \cos \theta_{\ell}-\int_{-1}^{0} \frac{d^{2} \Gamma}{d q^{2} d \cos \theta_{\ell}} d \cos \theta_{\ell}}{d \Gamma / d q^{2}} \\
& =\frac{b_{\ell}\left(q^{2}\right)}{d \Gamma / d q^{2}}=0 .
\end{aligned}
$$

Another observable of interest that we calculate is the longitudinal polarization asymmetry $P_{L}\left(q^{2}\right)$ of the leptons defined as [46]

$$
P_{L}\left(q^{2}\right)=\frac{1}{d \Gamma / d q^{2}}\left[\frac{d \Gamma^{h_{\ell}=-1}}{d q^{2}}-\frac{d \Gamma^{h_{\ell}=+1}}{d q^{2}}\right],
$$

where $h_{\ell}=+1(-1)$ implies a right-handed (left-handed) charged lepton $\ell^{-}$in the final state. For the $\bar{B}_{s} \rightarrow K \ell^{+} \ell^{-}$ decay, the lepton polarization is given by [46]

$P_{L}\left(q^{2}\right)=\frac{1}{3} \cdot \frac{G_{F}^{2} \alpha_{e m}^{2}\left|V_{t b} V_{t d}^{*}\right|^{2}}{256 \pi^{5} m_{B_{s}}^{3}} \cdot \frac{\beta_{\ell}^{2} \lambda^{3 / 2} \cdot \operatorname{Re}\left\{\delta_{V} \delta_{A}^{*}\right\}}{d \Gamma / d q^{2}}$.

For the $C P$-conjugated mode $B_{s}^{0} \rightarrow \bar{K} \ell^{+} \ell^{-}$, the decay amplitude and physical observables are obtained by making the substitution $\mathcal{A} \rightarrow \overline{\mathcal{A}}$, i.e., by making the complex conjugation of the CKM factor involved for the $\bar{B}_{s}^{0}$ decay modes. Analogous to Ref. [65], we also define the direct $C P$ asymmetry of the considered $B_{s} \rightarrow K l^{+} l^{-}$decays in the following form:

$$
\mathcal{A}_{C P}\left(q^{2}\right)=\frac{d \Gamma\left(\bar{B}_{s} \rightarrow K l^{+} l^{-}\right) / d q^{2}-d \bar{\Gamma}\left(B_{s} \rightarrow \bar{K} l^{+} l^{-}\right) / d q^{2}}{d \Gamma\left(\bar{B}_{s} \rightarrow K l^{+} l^{-}\right) / d q^{2}+d \bar{\Gamma}\left(B_{s} \rightarrow \bar{K} l^{+} l^{-}\right) / d q^{2}} .
$$

\section{B. Observables for $\boldsymbol{B}_{\boldsymbol{s}} \rightarrow \boldsymbol{K}^{*} \boldsymbol{\ell}^{+} \boldsymbol{\ell}^{-}$}

For a four body decay, $B_{s} \rightarrow K^{*}(\rightarrow \pi K) \ell^{+} \ell^{-}$, the decay distribution can be completely described in terms of four kinematic variables $[7,65,66]$ : the lepton invariant mass squared $\left(q^{2}\right)$ and three angles $\theta_{K^{*}}, \theta_{\ell}$, and $\phi$. The angle $\theta_{K^{*}}$ is the angle between the direction of flight of $K$ and the $B_{s}$ meson in the rest frame of $K^{*}, \theta_{\ell}$ is the angle made by $\ell^{-}$ with respect to the $B_{s}$ meson in the dilepton rest frame and $\phi$ is the azimuthal angle between the two planes formed by dilepton and $\pi K$. The full angular decay distribution of $\bar{B}_{s} \rightarrow K^{*}\left(\rightarrow \pi^{-} K^{+}\right) \ell^{+} \ell^{-}$is given by $[8,29,67]$,

$$
\frac{d^{4} \Gamma}{d q^{2} d \cos \theta_{K^{*}} d \cos \theta_{\ell} d \phi}=\frac{9}{32 \pi} I\left(q^{2}, \theta_{K^{*}}, \theta_{\ell}, \phi\right),
$$

where the functions $I\left(q^{2}, \theta_{K^{*}}, \theta_{\ell}, \phi\right)$ are of the following form [8]: 


$$
\begin{aligned}
I\left(q^{2}, \theta_{\ell}, \theta_{K^{*}}, \phi\right)= & \sum_{i} I_{i}\left(q^{2}\right) f_{i}\left(\theta_{K^{*}}, \theta_{\ell}, \phi\right) \\
= & I_{1}^{s} \sin ^{2} \theta_{K^{*}}+I_{1}^{c} \cos ^{2} \theta_{K^{*}}+\left(I_{2}^{s} \sin ^{2} \theta_{K^{*}}+I_{2}^{c} \cos ^{2} \theta_{K^{*}}\right) \cos 2 \theta_{\ell} \\
& +I_{3} \sin ^{2} \theta_{K^{*}} \sin ^{2} \theta_{\ell} \cos 2 \phi+I_{4} \sin 2 \theta_{K^{*}} \sin 2 \theta_{\ell} \cos \phi \\
& +I_{5} \sin 2 \theta_{K^{*}} \sin \theta_{\ell} \cos \phi+I_{6}^{s} \sin ^{2} \theta_{K^{*}} \cos \theta_{\ell}+I_{7} \sin 2 \theta_{K^{*}} \sin \theta_{\ell} \sin \phi \\
& +I_{8} \sin 2 \theta_{K^{*}} \sin 2 \theta_{\ell} \sin \phi+I_{9} \sin ^{2} \theta_{K^{*}} \sin ^{2} \theta_{\ell} \sin 2 \phi .
\end{aligned}
$$

For the $C P$-conjugated mode $B_{s} \rightarrow \bar{K}^{*}\left(\rightarrow \pi^{+} K^{-}\right) \ell^{+} \ell^{-}$, the corresponding expression of the angular decay distribution is

$$
\frac{d^{4} \bar{\Gamma}}{d q^{2} d \cos \theta_{K^{*}} d \cos \theta_{\ell} d \phi}=\frac{9}{32 \pi} \bar{I}\left(q^{2}, \theta_{K^{*}}, \theta_{\ell}, \phi\right) .
$$

The function $\bar{I}\left(q^{2}, \theta_{K^{*}}, \theta_{\ell}, \phi\right)$ is obtained from Eq. (64) by the substitution [8]:

$$
I_{1,2,3,4,7} \rightarrow \bar{I}_{1,2,3,4,7}, I_{5,6,8,9} \rightarrow-\bar{I}_{5,6,8,9}
$$

where $\bar{I}_{i}$ are obtained by making the complex conjugation for all weak phases in $I_{i}$. The minus sign in Eq. (66) is a result of the convention that, under the previous definitions of three angles, a $C P$ transformation interchanges the lepton and antilepton, leading to the modification $\theta_{\ell} \rightarrow$ $\theta_{\ell}-\pi$ and $\phi \rightarrow-\phi$.

The angular coefficients $I_{i}$ of the distributions in the above equation can be written in terms of the transverse amplitudes $[16,68]$. For the massless case there are six such complex amplitudes: $\mathcal{A}_{0}^{R, L}, \mathcal{A}_{\|}^{R, L}$, and $\mathcal{A}_{\perp}^{R, L}$. For the massive case, an additional complex amplitude $\mathcal{A}_{t}$ is required. In Table III, we show the expressions for those angular coefficients $I_{i}\left(q^{2}\right)$ and the corresponding angular factor $f_{i}\left(\theta_{K^{*}}, \theta_{\ell}, \phi\right)$ as those defined in Refs. [16,68].

The seven transversity amplitudes $\mathcal{A}_{0}^{R, L}, \mathcal{A}_{\|}^{R, L}, \mathcal{A}_{\perp}^{R, L}$, and

\begin{tabular}{|c|c|c|}
\hline$i$ & $I_{i}\left(q^{2}\right)$ & $f_{i}$ \\
\hline $1 s$ & $\left(\frac{3}{4}-\hat{m}_{\ell}^{2}\right)\left[\left|\mathcal{A}_{\|}^{\mathrm{L}}\right|^{2}+\left|\mathcal{A}_{\perp}^{\mathrm{L}}\right|^{2}+\left|\mathcal{A}_{\|}^{\mathrm{R}}\right|^{2}+\left|\mathcal{A}_{\perp}^{\mathrm{R}}\right|^{2}\right]+4 \hat{m}_{\ell}^{2} \operatorname{Re}\left[\mathcal{A}_{\perp}^{\mathrm{L}} \mathcal{A}_{\perp}^{\mathrm{R} *}+\mathcal{A}_{\|}^{\mathrm{L}} \mathcal{A}_{\|}^{\mathrm{R} *}\right]$ & $\sin ^{2} \theta_{K^{*}}$ \\
\hline $1 c$ & $\left|\mathcal{A}_{0}^{\mathrm{L}}\right|^{2}+\left|\mathcal{A}_{0}^{\mathrm{R}}\right|^{2}+4 \hat{m}_{\ell}^{2}\left[\left|\mathcal{A}_{t}\right|^{2}+2 \operatorname{Re}\left[\mathcal{A}_{0}^{\mathrm{L}} \mathcal{A}_{0}^{\mathrm{R} *}\right]\right]$ & $\cos ^{2} \theta_{K^{*}}$ \\
\hline $2 s$ & $\frac{1}{4} \beta_{\ell}^{2}\left[\left|\mathcal{A}_{\|}^{\mathrm{L}}\right|^{2}+\left|\mathcal{A}_{\perp}^{\mathrm{L}}\right|^{2}+\left|\mathcal{A}_{\|}^{\mathrm{R}}\right|^{2}+\left|\mathcal{A}_{\perp}^{\mathrm{R}}\right|^{2}\right]$ & $\sin ^{2} \theta_{K^{*}} \cos 2 \theta_{\ell}$ \\
\hline $2 c$ & $-\beta_{\ell}^{2}\left[\left|\mathcal{A}_{0}^{\mathrm{L}}\right|^{2}+\left|\mathcal{A}_{0}^{\mathrm{R}}\right|^{2}\right]$ & $\cos ^{2} \theta_{K^{*}} \cos 2 \theta_{\ell}$ \\
\hline 3 & $\frac{1}{2} \beta_{\ell}^{2}\left[\left|\mathcal{A}_{\perp}^{\mathrm{L}}\right|^{2}-\left|\mathcal{A}_{\|}^{\mathrm{L}}\right|^{2}+\left|\mathcal{A}_{\perp}^{\mathrm{R}}\right|^{2}-\left|\mathcal{A}_{\|}^{\mathrm{R}}\right|^{2}\right]$ & $\sin ^{2} \theta_{K^{*}} \sin ^{2} \theta_{\ell} \cos 2 \phi$ \\
\hline 4 & $\sqrt{\frac{1}{2}} \beta_{\ell}^{2} \operatorname{Re}\left(\mathcal{A}_{0}^{\mathrm{L}} \mathcal{A}_{\|}^{\mathrm{L} *}+\mathcal{A}_{0}^{\mathrm{R}} \mathcal{A}_{\|}^{\mathrm{R} *}\right)$ & $\sin 2 \theta_{K^{*}} \sin 2 \theta_{\ell} \cos \phi$ \\
\hline 5 & $\sqrt{2} \beta_{\ell} \operatorname{Re}\left(\mathcal{A}_{0}^{\mathrm{L}} \mathcal{A}_{\perp}^{\mathrm{L} *}-\mathcal{A}_{0}^{\mathrm{R}} \mathcal{A}_{\perp}^{\mathrm{R} *}\right)$ & $\sin 2 \theta_{K^{*}} \sin \theta_{\ell} \cos \phi$ \\
\hline $6 s$ & $2 \beta_{\ell} \operatorname{Re}\left(\mathcal{A}_{\|}^{\mathrm{L}} \mathcal{A}_{\perp}^{\mathrm{L} *}-\mathcal{A}_{\|}^{\mathrm{R}} \mathcal{A}_{\perp}^{\mathrm{R} *}\right)$ & $\sin ^{2} \theta_{K^{*}} \cos \theta_{\ell}$ \\
\hline 7 & $\sqrt{2} \beta_{\ell} \operatorname{Im}\left(\mathcal{A}_{0}^{\mathrm{L}} \mathcal{A}_{\|}^{\mathrm{L} *}-\mathcal{A}_{0}^{\mathrm{R}} \mathcal{A}_{\|}^{\mathrm{R} *}\right)$ & $\sin 2 \theta_{K^{*}} \sin \theta_{\ell} \sin \phi$ \\
\hline 8 & $\sqrt{\frac{1}{2}} \beta_{\ell}^{2} \operatorname{Im}\left(\mathcal{A}_{0}^{\mathrm{L}} \mathcal{A}_{\perp}^{\mathrm{L} *}+\mathcal{A}_{0}^{\mathrm{R}} \mathcal{A}_{\perp}^{\mathrm{R} *}\right)$ & $\sin 2 \theta_{K^{*}} \sin 2 \theta_{\ell} \sin \phi$ \\
\hline 9 & $\beta_{\ell}^{2} \operatorname{Im}\left(\mathcal{A}_{\|}^{\mathrm{L} *} \mathcal{A}_{\perp}^{\mathrm{L}}+\mathcal{A}_{\|}^{\mathrm{R} *} \mathcal{A}_{\perp}^{\mathrm{R}}\right)$ & $\sin ^{2} \theta_{K^{*}} \sin ^{2} \theta_{\ell} \sin 2 \phi$ \\
\hline
\end{tabular}
$\mathcal{A}_{t}$, in turn, can be expressed in terms of the relevant $B_{s} \rightarrow$ $K^{*} \ell^{+} \ell^{-}$form factors $[6,8]$ :

$$
\begin{gathered}
\mathcal{A}_{\perp}^{\mathrm{L}, \mathrm{R}}=-N_{\ell} \sqrt{2 N_{K^{*}}} \sqrt{\lambda}\left[\left(C_{9}^{\mathrm{eff}} \mp C_{10}\right) \frac{V\left(q^{2}\right)}{m_{B_{s}}+m_{K^{*}}}+2 \hat{m}_{b} C_{7}^{\mathrm{eff}} T_{1}\left(q^{2}\right)\right], \\
\mathcal{A}_{\|}^{\mathrm{L}, \mathrm{R}}=N_{\ell} \sqrt{2 N_{K^{*}}}\left[\left(C_{9}^{\mathrm{eff}} \mp C_{10}\right)\left(m_{B_{s}}+m_{K^{*}}\right) A_{1}\left(q^{2}\right)+2 \hat{m}_{b} C_{7}^{\mathrm{eff}}\left(m_{B_{s}}^{2}-m_{K^{*}}^{2}\right) T_{2}\left(q^{2}\right)\right],
\end{gathered}
$$

TABLE III. The explicit expressions of the angular coefficients $I_{i}\left(q^{2}\right)$ and $f_{i}$ that appeared in Eq. (64). 


$$
\begin{gathered}
\mathcal{A}_{0}^{\mathrm{L}, \mathrm{R}}=\frac{N_{\ell} \sqrt{N_{K^{*}}}}{2 m_{K^{*}} \sqrt{q^{2}}}\left\{\left(C_{9}^{\mathrm{eff}} \mp C_{10}\right)\left[\left(m_{B_{s}}^{2}-m_{K^{*}}^{2}-q^{2}\right)\left(m_{B_{s}}+m_{K^{*}}\right) A_{1}\left(q^{2}\right)-\frac{\lambda}{m_{B_{s}}+m_{K^{*}}} A_{2}\left(q^{2}\right)\right]\right. \\
\left.+2 m_{b} C_{7}^{\mathrm{eff}}\left[\left(m_{B_{s}}^{2}+3 m_{K^{*}}^{2}-q^{2}\right) T_{2}\left(q^{2}\right)-\frac{\lambda}{m_{B_{s}}^{2}-m_{K^{*}}^{2}} T_{3}\left(q^{2}\right)\right]\right\}, \\
\mathcal{A}_{t}=2 N_{\ell} \sqrt{N_{K^{*}}} \frac{\sqrt{\lambda}}{\sqrt{q^{2}}} C_{10} A_{0}\left(q^{2}\right),
\end{gathered}
$$

where the factors $N_{\ell}$ and $N_{K^{*}}$ are of the following form:

$$
N_{\ell}=\frac{i \alpha_{e m} G_{F}}{4 \sqrt{2} \pi} V_{t b} V_{t d}^{*}, \quad N_{K^{*}}=\frac{8 \beta_{\ell} \sqrt{\lambda} q^{2}}{3 \times 256 \pi^{3} m_{B_{s}^{3}}^{3}},
$$

with $\lambda \equiv\left(m_{B_{s}}^{2}-m_{K^{*}}^{2}-q^{2}\right)^{2}-4 m_{K^{*}}^{2} q^{2}, \beta_{\ell}=\sqrt{1-4 m_{\ell}^{2} / q^{2}}$, and $\hat{m}_{b}=m_{b} / q^{2}$.

Analogous to Ref. [69], one can write down three partially integrated decay distributions, integrating all but one angle at a time. For the $C P$-conjugated process, we can do the similar operation using the corresponding decay distributions.

(1) The $\theta_{K^{*}}$ distribution:

$$
\begin{aligned}
\frac{d^{2} \Gamma}{d q^{2} d \cos \theta_{K^{*}}} & =a_{\theta_{K^{*}}}\left(q^{2}\right)+c_{\theta_{K^{*}}}\left(q^{2}\right) \cos ^{2} \theta_{K^{*}}, \\
a_{\theta_{K^{*}}}\left(q^{2}\right) & =\frac{3}{8}\left(3 I_{1}^{s}-I_{2}^{s}\right), \\
c_{\theta_{K^{*}}}\left(q^{2}\right) & =\frac{3}{8}\left(3 I_{1}^{c}-3 I_{1}^{s}-I_{2}^{c}+I_{2}^{s}\right) .
\end{aligned}
$$

(2) The $\theta_{\ell}$ distribution:

$$
\begin{aligned}
\frac{d^{2} \Gamma}{d q^{2} d \cos \theta_{\ell}}= & a_{\theta_{\ell}}\left(q^{2}\right)+b_{\theta_{\ell}}\left(q^{2}\right) \cos \theta_{\ell} \\
& +c_{\theta_{\ell}}\left(q^{2}\right) \cos ^{2} \theta_{\ell}, \\
a_{\theta_{\ell}}\left(q^{2}\right)= & \frac{3}{8}\left(I_{1}^{c}+2 I_{1}^{s}-I_{2}^{c}-2 I_{2}^{s}\right), \\
b_{\theta_{\ell}}\left(q^{2}\right)= & \frac{3}{4}\left(I_{6}^{s}\right), \\
c_{\theta_{\ell}}\left(q^{2}\right)= & \frac{3}{4}\left(I_{2}^{c}+2 I_{2}^{s}\right) .
\end{aligned}
$$

(3) The $\phi$ distribution:

$$
\begin{aligned}
\frac{d^{2} \Gamma}{d q^{2} d \phi} & =a_{\phi}\left(q^{2}\right)+c_{\phi}^{c}\left(q^{2}\right) \cos 2 \phi+c_{\phi}^{s}\left(q^{2}\right) \sin 2 \phi \\
a_{\phi}\left(q^{2}\right) & =\frac{1}{8 \pi}\left(3 I_{1}^{c}+6 I_{1}^{s}-I_{2}^{c}-2 I_{2}^{s}\right), \\
c_{\phi}^{c}\left(q^{2}\right) & =\frac{1}{2 \pi} I_{3}, \\
c_{\phi}^{s}\left(q^{2}\right) & =\frac{1}{2 \pi} I_{9} .
\end{aligned}
$$

From the full angular distributions as defined in Eq. (64), we set various coefficients apart and combine them into diverse quantities normalized to the differential decay rate and other observables [69]. Analogous observables are constructed for the $C P$-conjugated mode, after making the replacements as shown in Eq. (65) and (66).

(1) The differential decay rate:

$$
\frac{d \Gamma}{d q^{2}}=\frac{1}{4}\left(3 I_{1}^{c}+6 I_{1}^{s}-I_{2}^{c}-2 I_{2}^{s}\right)
$$

(2) The lepton forward-backward asymmetry:

$$
\mathcal{A}_{\mathrm{FB}}\left(q^{2}\right)=\frac{b_{\theta_{\ell}}\left(q^{2}\right)}{d \Gamma / d q^{2}}=\frac{3 I_{6}^{s}}{3 I_{1}^{c}+6 I_{1}^{s}-I_{2}^{c}-2 I_{2}^{s}},
$$

(3) The $K^{*}$ polarization fraction:

$$
R_{L, T}\left(q^{2}\right)=\frac{d \Gamma_{L} / d q^{2}}{d \Gamma_{T} / d q^{2}}
$$

where $\Gamma_{L}$ and $\Gamma_{T}$ represent the longitudinal and transverse $K^{*}$ polarization decay rates,

$$
\begin{gathered}
\frac{d \Gamma_{L}}{d q^{2}}=\frac{2}{3}\left[a_{\theta_{K^{*}}}\left(q^{2}\right)+c_{\theta_{K^{*}}}\left(q^{2}\right)\right]=\frac{1}{4}\left(3 I_{1}^{c}-I_{2}^{c}\right), \\
\frac{d \Gamma_{T}}{d q^{2}}=\frac{4}{3} a_{\theta_{K^{*}}}\left(q^{2}\right)=\frac{1}{2}\left(3 I_{1}^{s}-I_{2}^{s}\right) .
\end{gathered}
$$

Alternatively, one can define the quantity $F_{L}^{K^{*}}$ which is a measure of the longitudinally polarized $K^{*}$ 's in the whole ensemble of $B_{s} \rightarrow K^{*} \ell \ell$ decays, which is linked to $R_{L, T}\left(q^{2}\right)$ as

$$
\begin{aligned}
F_{L}^{K^{*}}\left(q^{2}\right) & =\frac{R_{L, T}\left(q^{2}\right)}{1+R_{L, T}\left(q^{2}\right)}=\frac{1}{4} \cdot \frac{3 I_{1}^{c}-I_{2}^{c}}{d \Gamma / d q^{2}} \\
& =\frac{3 I_{1}^{c}-I_{2}^{c}}{3 I_{1}^{c}+6 I_{1}^{s}-I_{2}^{c}-2 I_{2}^{s}},
\end{aligned}
$$

where $F_{L}^{K^{*}}$ is a number obtained by integrating $F_{L}^{K^{*}}\left(q^{2}\right)$ over the proper phase space.

(4) The direct $C P$ asymmetry can also be defined in the same way as for $B_{s} \rightarrow K l^{+} l^{-}$decays: 


$$
\mathcal{A}_{C P}\left(q^{2}\right)=\frac{d \Gamma\left(\bar{B}_{s} \rightarrow K^{*} l^{+} l^{-}\right) / d q^{2}-d \bar{\Gamma}\left(B_{s} \rightarrow \bar{K}^{*} l^{+} l^{-}\right) / d q^{2}}{d \Gamma\left(\bar{B}_{s} \rightarrow K^{*} l^{+} l^{-}\right) / d q^{2}+d \bar{\Gamma}\left(B_{s} \rightarrow \bar{K}^{*} l^{+} l^{-}\right) / d q^{2}} .
$$

The above observables are constructed from Eqs. (63) and (64) by integrating over the angles in various ranges. These observables which have a form factor dependence in the leading order are called form factor dependent observables and generally plagued by the large uncertainties of the form factors. To avoid this problem, a lot of work has been done to construct observables which are theoretically clean in the low $-q^{2}$ region. Such observables are free from this dependence at the leading order and are called form factor independent (FFI) observables. In this paper, we study both kinds of observables.

As a necessary and sufficient condition, such FFI observable must be invariant under the symmetry transformations of the transverse amplitudes $A$ 's; we then say that the observable respects the symmetries of the angular distribution. Fortunately, there exists a systematic procedure to construct all such possible observables as discussed in Ref. [68].

We start defining the following complex vectors [70]:

$$
n_{\|}=\left(\begin{array}{c}
A_{\|}^{L} \\
A_{\|}^{R *}
\end{array}\right), \quad n_{\perp}=\left(\begin{array}{c}
A_{\perp}^{L} \\
-A_{\perp}^{R *}
\end{array}\right), \quad n_{0}=\left(\begin{array}{c}
A_{0}^{L} \\
A_{0}^{R *}
\end{array}\right) .
$$

With these vectors we can construct the products $\left|n_{i}\right|^{2}=$ $n_{i}^{\dagger} n_{i}$ and $n_{i}^{\dagger} n_{j}$,

$$
\begin{array}{cc}
\left|n_{\|}\right|^{2}=\left|A_{\|}^{L}\right|^{2}+\left|A_{\|}^{R}\right|^{2}=\frac{2 I_{2}^{s}-I_{3}}{\beta_{\ell}^{2}}, & n_{\perp}^{\dagger} n_{\|}=A_{\perp}^{L *} A_{\|}^{L}-A_{\perp}^{R} A_{\|}^{R *}=\frac{\beta_{\ell} I_{6}^{s}-2 i I_{9}}{2 \beta_{\ell}^{2}}, \\
\left|n_{\perp}\right|^{2}=\left|A_{\perp}^{L}\right|^{2}+\left|A_{\perp}^{R}\right|^{2}=\frac{2 I_{2}^{s}+I_{3}}{\beta_{\ell}^{2}}, & n_{0}^{\dagger} n_{\|}=A_{0}^{L *} A_{\|}^{L}+A_{0}^{R} A_{\|}^{R *}=\frac{2 I_{4}-i \beta_{\ell} I_{7}}{\sqrt{2} \beta_{\ell}^{2}}, \\
\left|n_{0}\right|^{2}=\left|A_{0}^{L}\right|^{2}+\left|A_{0}^{R}\right|^{2}=-\frac{I_{2}^{c}}{\beta_{\ell}^{2}}, & n_{0}^{\dagger} n_{\perp}=A_{0}^{L *} A_{\perp}^{L}-A_{0}^{R} A_{\perp}^{R *}=\frac{\beta_{\ell} I_{5}-2 i I_{8}}{\sqrt{2} \beta_{\ell}^{2}} .
\end{array}
$$

We examine the following (clean) FFI observables [68]:

$$
\begin{gathered}
P_{1}=\frac{\left|n_{\perp}\right|^{2}-\left|n_{\|}\right|^{2}}{\left|n_{\perp}\right|^{2}+\left|n_{\|}\right|^{2}}=\frac{I_{3}}{2 I_{2}^{s}}, \quad P_{2}=\frac{\operatorname{Re}\left(n_{\perp}^{\dagger} n_{\|}\right)}{\left|n_{\|}\right|^{2}+\left|n_{\perp}\right|^{2}}=\beta_{\ell} \frac{I_{6}^{s}}{8 I_{2}^{s}}, \\
P_{3}=\frac{\operatorname{Im}\left(n_{\perp}^{\dagger} n_{\|}\right)}{\left|n_{\|}\right|^{2}+\left|n_{\perp}\right|^{2}}=-\frac{I_{9}}{4 I_{2}^{s}}, \quad P_{4}=\frac{\operatorname{Re}\left(n_{0}^{\dagger} n_{\|}\right)}{\sqrt{\left|n_{\|}\right|^{2}\left|n_{0}\right|^{2}}}=\frac{\sqrt{2} I_{4}}{\sqrt{-I_{2}^{c}\left(2 I_{2}^{s}-I_{3}\right)}}, \\
P_{5}=\frac{\operatorname{Re}\left(n_{0}^{\dagger} n_{\perp}\right)}{\sqrt{\left|n_{\perp}\right|^{2}\left|n_{0}\right|^{2}}}=\frac{\beta_{\ell} I_{5}}{\sqrt{-2 I_{2}^{c}\left(2 I_{2}^{s}+I_{3}\right)}}, \\
P_{6}=\frac{\operatorname{Im}\left(n_{0}^{\dagger} n_{\|}\right)}{\sqrt{\left|n_{\|}\right|^{2}\left|n_{0}\right|^{2}}}=-\frac{\beta_{\ell} I_{7}}{\sqrt{-2 I_{2}^{c}\left(2 I_{2}^{s}-I_{3}\right)}}, \\
P_{8}=\frac{\operatorname{Im}\left(n_{0}^{\dagger} n_{\perp}\right)}{\sqrt{\left|n_{\perp}\right|^{2}\left|n_{0}\right|^{2}}}=-\frac{\sqrt{2} I_{8}}{\sqrt{-I_{2}^{c}\left(2 I_{2}^{s}+I_{3}\right)}} .
\end{gathered}
$$

The primed observables are also defined in the following form [29]:

$$
\begin{array}{cc}
P_{4}^{\prime} \equiv P_{4} \sqrt{1-P_{1}}=\frac{I_{4}}{\sqrt{-I_{2}^{c} I_{2}^{s}}}, \quad P_{5}^{\prime} \equiv P_{5} \sqrt{1+P_{1}}=\frac{\beta_{\ell} I_{5}}{2 \sqrt{-I_{2}^{c} I_{2}^{s}}}, \\
P_{6}^{\prime} \equiv P_{6} \sqrt{1-P_{1}}=-\frac{\beta_{\ell} I_{7}}{2 \sqrt{-I_{2}^{c} I_{2}^{s}}}, & P_{8}^{\prime} \equiv P_{8} \sqrt{1+P_{1}}=-\frac{I_{8}}{\sqrt{-I_{2}^{c} I_{2}^{s}}} .
\end{array}
$$


These primed observables $P_{4,5,6,8}^{\prime}$ are clean and good approximations to $P_{4,5,6,8}$ due to the fact that $P_{1} \simeq 0$ in the SM. From the experimental perspective, fitting the primed observables will be simpler and more efficient despite the fact that the whole analysis can be performed directly in terms of the observables $P_{4,5,6,8}$.

Since most observables are written in terms of the ratios, $O_{i}^{\ell}\left(q^{2}\right)=\mathcal{N}_{i}^{\ell}\left(q^{2}\right) / \mathcal{D}_{i}^{\ell}\left(q^{2}\right)$ with $\mathcal{N}$ and $\mathcal{D}$ being generically a numerator and a denominator, the integrated quantities are defined as in Ref. [69]:

$$
\left\langle O_{i}^{\ell}\right\rangle=\frac{\int_{4 m_{\ell}^{2}}^{q_{\max }^{2}} \mathcal{N}_{i}^{\ell}\left(q^{2}\right) d q^{2}}{\int_{4 m_{\ell}^{2}}^{q_{\max }^{2}} \mathcal{D}_{i}^{\ell}\left(q^{2}\right) d q^{2}} .
$$

We also check the physical observables $R_{K, K^{*}}^{e \mu}$ and $R_{K, K^{*}}^{\mu \tau}$, as defined in Eqs. (3) and (4), since the theoretical uncertainties are largely canceled in the ratio of the branching ratios of $B_{s} \rightarrow K^{(*)} \ell^{+} \ell^{-}$decays.
In the region $q^{2}<4 m_{\mu}^{2}$, where only the $e^{+} e^{-}$modes are allowed, there is a large enhancement of $B_{s} \rightarrow K^{*} e^{+} e^{-}$due to the $1 / q^{2}$ scaling of the photon penguin contribution [71]. In order to remove the phase space effects in the ratio $R_{K^{*}}^{e \mu}$ and keep consistent with the other analysis [72], here we also use the lower cut of $4 m_{\mu}^{2}$ for both the electron and muon modes in the definition of the ratio $R_{K^{*}}^{e \mu}$ as in Ref. [72]:

$$
R_{K^{*}}^{e \mu}=\frac{\int_{4 m_{\mu}^{2}}^{q_{\max }^{2}} d q^{2} \frac{d \mathcal{B}\left(B_{s} \rightarrow K^{*} \mu^{+} \mu^{-}\right)}{d q^{2}}}{\int_{4 m_{\mu}^{2}}^{q_{\max }^{2}} d q^{2} \frac{d \mathcal{B}\left(B_{s} \rightarrow K^{*} e^{+} e^{-}\right)}{d q^{2}}} .
$$

\section{NUMERICAL RESULTS AND DISCUSSIONS}

In the numerical calculations we use the following input parameters (here masses and decay constants are in units of $\mathrm{GeV})[30,31]$ :

$$
\begin{aligned}
& \Lambda_{\overline{\mathrm{MS}}}^{f=4}=0.250, \quad \tau_{B_{s}^{0}}=1.509 \mathrm{ps}, \quad m_{b}=4.8, \quad m_{W}=80.38, \\
& m_{K}=0.498, \quad m_{K^{*}}=0.892, \quad m_{B_{s}}=5.367, \quad m_{\tau}=1.777, \\
& f_{B_{s}}=0.23, \quad f_{K}=0.16, \quad f_{K^{*}}=0.217, \quad f_{K^{*}}^{T}=0.185,
\end{aligned}
$$

For the CKM matrix elements and angles, we use the values as given in PDG 2018 [30]:

$$
\begin{aligned}
& V_{t b}=1.019 \pm 0.025, \quad V_{u d}=0.97420 \pm 0.00021, \\
& V_{t d}=\left|V_{t d}\right| \cdot e^{-i \beta}, \quad\left|V_{t d}\right|=(8.1 \pm 0.5) \times 10^{-3}, \quad \sin (2 \beta)=0.691 \pm 0.017, \\
& V_{u b}=\left|V_{u b}\right| \cdot e^{-i \gamma}, \quad\left|V_{u b}\right|=(3.94 \pm 0.36) \times 10^{-3}, \quad \gamma=\left(73.5_{-5.1}^{+4.2}\right)^{\circ} .
\end{aligned}
$$

\section{A. The form factors}

For the considered semileptonic decays, the differential decay rates and other physical observables strongly rely on the value and the shape of the relevant form factors $F_{0,+}\left(q^{2}\right)$ and $F_{T}\left(q^{2}\right)$ for $B_{s} \rightarrow K \ell^{+} \ell^{-}$decays, and the form factors $V\left(q^{2}\right), A_{0,1,2}\left(q^{2}\right)$ and $T_{1,2,3}\left(q^{2}\right)$ for $B_{s} \rightarrow$ $K^{*} \ell^{+} \ell^{-}$decays. These form factors have been calculated in rather different theories or models, such as the relativistic quark model (RQM) [73], the LCSR [28,74], and the covariant confined quark model (CCQM) [75]. For the heavy $B / B_{s}$ to light meson (such as $K, \pi, \eta^{\prime}, \rho, K^{*}$, etc.) transitions, on the other hand, the relevant form factors at the low- $q^{2}$ region have been evaluated successfully by employing the PQCD factorization approach, for example, in Refs. [26,27,33-35,41,42,76].

Since the PQCD predictions for the considered form factors are reliable only at the low- $q^{2}$ region, we usually calculate explicitly the values of the relevant form factors at the low- $q^{2}$ region, say $0 \leq q^{2} \leq m_{\tau}^{2}$, and then make an extrapolation for all relevant form factors from the low- $q^{2}$ region to the large- $q^{2}$ region by using the pole model parametrization $[77,78]$ or other different methods.
In Refs. [79-81], we developed a new method: the socalled PQCD + Lattice approach. Here we still use the PQCD approach to evaluate the form factors at the low- $q^{2}$ region, but take those currently available lattice QCD results for the relevant form factors at the high- $q^{2}$ region as the lattice QCD input to improve the extrapolation of the form factors up to $q_{\max }^{2}$. In Refs. [80,81], we used the BCL parametrization method $[36,37]$ instead of the traditional pole model parametrization since the BCL method has better convergence.

In Tables IV and V, we list the values of the lattice QCD results for the relevant $B_{s} \rightarrow K^{*}$ transition form factors at

TABLE IV. The values for the lattice QCD results of the relevant $B_{s} \rightarrow K$ transition form factors at three reference points of $q^{2}$ : $q^{2}=17.9,21.2 \mathrm{GeV}^{2}$ and $q_{\max }^{2}=\left(m_{B_{s}}-m_{K}\right)^{2} \approx 23.8 \mathrm{GeV}^{2}[82]$.

\begin{tabular}{cccc}
\hline \hline & \multicolumn{3}{c}{$q^{2}$ Point } \\
\cline { 2 - 4 }$F F$ & 17.9 & \multicolumn{1}{c}{21.2} & \multicolumn{1}{c}{23.8} \\
\hline$F_{0}\left(q^{2}\right)$ & $0.48(5)$ & $0.63(5)$ & $0.80(5)$ \\
$F_{+}\left(q^{2}\right)$ & $0.98(7)$ & $1.64(10)$ & $2.76(16)$ \\
\hline \hline
\end{tabular}


TABLE V. The values for the lattice QCD results of the relevant $B_{s} \rightarrow K^{*}$ transition form factors at three reference points of $q^{2}$ : $q^{2}=12,16 \mathrm{GeV}^{2}$ and $q_{\max }^{2}=\left(m_{B_{s}}-m_{K^{*}}\right)^{2} \approx 20 \mathrm{GeV}^{2}$ [83].

\begin{tabular}{llllllll}
$q^{2}$ & $V\left(q^{2}\right)$ & $A_{0}\left(q^{2}\right)$ & $A_{1}\left(q^{2}\right)$ & $A_{2}\left(q^{2}\right)$ & $T_{1}\left(q^{2}\right)$ & $T_{2}\left(q^{2}\right)$ & $T_{3}\left(q^{2}\right)$ \\
\hline \hline 2 & $0.56(9)$ & $0.84(9)$ & $0.37(3)$ & $0.46(3)$ & $0.61(4)$ & $0.39(3)$ & $0.43(4)$
\end{tabular}

$\begin{array}{lllllllll}12 & 0.56(9) & 0.84(9) & 0.37(3) & 0.46(3) & 0.61(4) & 0.39(3) & 0.43(4)\end{array}$

$\begin{array}{llllllll}16 & 1.02(8) & 1.33(8) & 0.45(3) & 0.60(5) & 0.90(6) & 0.47(3) & 0.67(5)\end{array}$ $201.99(13) 2.38(16) 0.58(3) 0.85(12) 1.48(10) 0.60(3) 1.10(7)$

three reference points of $q^{2}[82,83]$ used in this paper. The systematic uncertainties are included.

In this work, we will use both the PQCD factorization approach and the PQCD + Lattice approach to evaluate all relevant form factors over the whole range of $q^{2}$.

(1) In the PQCD approach, we use the definitions and formulas as given in Eqs. (38)-(42), and (44)-(50) to calculate the values of all relevant form factors $F_{0,+, T}\left(q^{2}\right), V\left(q^{2}\right), A_{0,1,2}\left(q^{2}\right)$, and $T_{1,2,3}\left(q^{2}\right)$ in the low- $q^{2}$ region: $0 \leq q^{2} \leq m_{\tau}^{2}$. We then make the extrapolation for these form factors to the large- $q^{2}$ region up to $q_{\max }^{2}$ by using the proper parametrization method.

(2) In the PQCD + Lattice approach, we take the lattice QCD results for the form factors at some large- $q^{2}$ points as inputs and then make a combined fit to the PQCD and the lattice QCD results at the low- and high $q^{2}$-region.

(3) For both approaches, we always use the same $z$-series parametrization as in Refs. $[29,74]$ to make the extrapolation:

$$
z\left(q^{2}\right)=\frac{\sqrt{t_{+}-q^{2}}-\sqrt{t_{+}-t_{0}}}{\sqrt{t_{+}-q^{2}}+\sqrt{t_{+}-t_{0}}}
$$

where $\quad t_{ \pm}=\left(m_{B_{s}} \pm m_{K^{(*)}}\right)^{2} \quad$ and $\quad t_{0}=t_{+}(1-$ $\left.\sqrt{1-t_{-} / t_{+}}\right)$. Form factors are parametrized as

$$
F_{i}\left(q^{2}\right)=P_{i}\left(q^{2}\right) \Sigma_{k} \alpha_{k}^{i}\left[z\left(q^{2}\right)-z(0)\right]^{k},
$$

where $P_{i}\left(q^{2}\right)=\left(1-q^{2} / m_{R, i}^{2}\right)^{-1}$ is a simple pole corresponding to the $\bar{d} b$ resonance with appropriate $J^{P}$ in the spectrum and $m_{R, i}$ is the resonance mass: $m_{R} \rightarrow \infty$ for $F_{0}\left(q^{2}\right)$ (no pole), $5.279 \mathrm{GeV}$ for $A_{0}\left(q^{2}\right)$ couple to $B\left(0^{-}\right), 5.325 \mathrm{GeV}$ for $F_{+, T}\left(q^{2}\right)$, $V\left(q^{2}\right)$ and $T_{1}\left(q^{2}\right)$ couple to $B^{*}\left(1^{-}\right)$, and $5.724 \mathrm{GeV}$ for the rest of the form factors couple to $B_{1}\left(1^{+}\right)$.

In Table VI, as a comparison, we show the center values of all relevant form factors in this work and other theoretical predictions as given in Refs. [26,28,44,73,84-89] at the scale $q^{2}=0$. The PQCD factorization approach is applied in Refs. [44,86] and in Ref. [26] with the inclusion of the NLO corrections. The CCQM is used in Ref. [75]. Calculations are based on LCSRs in Refs. [28,74] with hadronic input parameters and in Ref. [84] with the inclusion of the one-loop radiative corrections. In Ref. [73], the authors used the relativistic quark model based on the quasipotential approach. In Ref. [85], the authors used the quark model and relativistic dispersion approach. In Ref. [87], the light-cone quark model is utilized based on the basis of the soft collinear effective theory. The authors of Ref. [88] employed the LCSR in the framework of the heavy quark effective theory. In Ref. [89], the authors evaluated the transition form factors in the six-quark effective Hamiltonian approach. One can see that there is no significant difference between the theoretical predictions for the $B_{s} \rightarrow K^{(*)}$ transition form factors evaluated at $q^{2}=0$ in various models or approaches.

In Table VII, we list the PQCD predictions for the form factors $F_{+, 0, T}\left(q^{2}\right), V\left(q^{2}\right), A_{0,1,2}\left(q^{2}\right)$, and $T_{1,2,3}\left(q^{2}\right)$ with the corresponding pole and resonance masses, and the fitting parametrization constants $\left(\alpha_{0}, \alpha_{1}, \alpha_{2}\right)$ in Eq. (98) for $B_{s} \rightarrow K^{(*)}$ transitions. It is simple to figure out the relation $F_{i}(0)=\alpha_{0}^{i}$ by substituting $q^{2}$ with zero on both sides of Eq. (98). The theoretical errors of the form factors as

TABLE VI. The theoretical predictions for the center values of the form factors of the $B_{s} \rightarrow K^{(*)}$ transitions at $q^{2}=0$ obtained by using rather different theories or models.

\begin{tabular}{lcccccccc}
\hline \hline & $F_{0,+}(0)$ & $F_{T}(0)$ & $V(0)$ & $A_{0}(0)$ & $A_{1}(0)$ & $A_{2}(0)$ & $T_{1,2}(0)$ & $T_{3}(0)$ \\
\hline This paper & 0.22 & 0.22 & 0.24 & 0.21 & 0.19 & 0.19 & 0.21 & 0.16 \\
PQCD[26] & 0.26 & 0.28 & $\ldots$ & $\ldots$ & $\ldots$ & $\ldots$ & $\ldots$ & $\ldots$ \\
PQCD[86] & $\ldots$ & $\ldots$ & 0.20 & 0.24 & 0.15 & 0.11 & 0.18 & 0.16 \\
PQCD[44] & 0.24 & $\ldots$ & 0.21 & 0.25 & 0.16 & $\ldots$ & $\ldots$ & $\ldots$ \\
CCQM[75] & $\ldots$ & $\ldots$ & 0.24 & 0.18 & 0.21 & 0.21 & 0.21 & 0.14 \\
LCSR[28] & 0.336 & 0.320 & $\ldots$ & $\ldots$ & $\ldots$ & $\ldots$ & $\ldots$ & $\ldots$ \\
LCSR[74] & $\ldots$ & $\ldots$ & 0.296 & 0.314 & 0.230 & $\ldots$ & 0.239 & $\ldots$ \\
LCSR[84] & 0.30 & $\ldots$ & 0.311 & 0.360 & 0.233 & 0.181 & 0.260 & 0.136 \\
RQM[73] & 0.284 & 0.236 & 0.291 & 0.289 & 0.287 & 0.286 & 0.238 & 0.122 \\
RDA[85] & 0.31 & 0.31 & 0.38 & 0.37 & 0.29 & 0.26 & 0.32 & 0.23 \\
SCET[87] & 0.290 & 0.317 & 0.323 & 0.279 & 0.232 & 0.210 & 0.271 & 0.165 \\
HQEFT[88] & 0.296 & 0.288 & 0.285 & 0.222 & 0.227 & 0.183 & 0.251 & 0.169 \\
SQEH[89] & 0.260 & $\ldots$ & 0.227 & 0.280 & 0.178 & $\ldots$ & $\ldots$ & $\ldots$ \\
\hline \hline
\end{tabular}


TABLE VII. The PQCD predictions for the form factors of $B_{s} \rightarrow K^{(*)}$ transitions. Form factors $F_{+, 0, T}\left(q^{2}\right), V\left(q^{2}\right)$, $A_{0,1,2}\left(q^{2}\right)$, and $T_{1,2,3}\left(q^{2}\right)$ are fitted by using Eq. (98).

\begin{tabular}{llccc}
\hline \hline PQCD & $B\left(J^{P}\right)$ & $\alpha_{0}$ & $\alpha_{1}$ & $\alpha_{2}$ \\
\hline$F_{+}^{B_{s} \rightarrow K}$ & $B^{*}\left(1^{-}\right)$ & $0.22_{-0.06}^{+0.04}\left(\omega_{B_{s}}\right) \pm 0.005\left(a_{i}^{K}\right)$ & $-1.21_{-0.23}^{+0.36} \pm 0.02$ & $0.06_{-1.09}^{+1.74} \pm 0.14$ \\
$F_{0}^{B_{s}} \rightarrow K$ & no pole & $0.22_{-0.06}^{+0.04}\left(\omega_{B_{s}}\right) \pm 0.005\left(a_{i}^{K}\right)$ & $-1.31_{-0.24}^{+0.40} \pm 0.03$ & $-0.22_{-1.11}^{+1.73} \pm 0.13$ \\
$F_{T}^{B_{s} \rightarrow K}$ & $B^{*}\left(1^{-}\right)$ & $0.22_{-0.06}^{+0.04}\left(\omega_{B_{s}}\right) \pm 0.005\left(a_{i}^{K}\right)$ & $-1.37_{-0.29}^{+0.40} \pm 0.03$ & $7.05_{-1.08}^{+1.85} \pm 0.15$ \\
$V^{B_{s} \rightarrow K^{*}}$ & $B^{*}\left(1^{-}\right)$ & $0.24_{-0.04}^{+0.05}\left(\omega_{B_{s}}\right) \pm 0.005\left(a_{i}^{K^{*}}\right)$ & $-1.87_{-0.42}^{+0.31} \pm 0.03$ & $7.56_{-1.41}^{+1.22} \pm 0.13$ \\
$A_{0}^{B_{s} \rightarrow K^{*}}$ & $B^{0}\left(0^{-}\right)$ & $0.21_{-0.03}^{+0.04}\left(\omega_{B_{s}}\right) \pm 0.003\left(a_{i}^{K^{*}}\right)$ & $-1.43_{-0.29}^{+0.25} \pm 0.02$ & $8.28_{-1.03}^{+1.38} \pm 0.11$ \\
$A_{1}^{B_{s} \rightarrow K^{*}}$ & $B_{1}\left(1^{+}\right)$ & $0.19_{-0.03}^{+0.04}\left(\omega_{B_{s}}\right) \pm 0.003\left(a_{i}^{K^{*}}\right)$ & $-0.64_{-0.14}^{+0.13} \pm 0.01$ & $0.35_{-1.02}^{+1.04} \pm 0.10$ \\
$A_{2}^{B_{s} \rightarrow K^{*}}$ & $B_{1}\left(1^{+}\right)$ & $0.19_{-0.03}^{+0.04}\left(\omega_{B_{s}}\right) \pm 0.003\left(a_{i}^{K^{*}}\right)$ & $-1.42_{-0.31}^{+0.28} \pm 0.03$ & $2.77_{-1.07}^{+1.68} \pm 0.12$ \\
$T_{1}^{B_{s} \rightarrow K^{*}}$ & $B^{*}\left(1^{-}\right)$ & $0.21_{-0.03}^{+0.04}\left(\omega_{B_{s}}\right) \pm 0.003\left(a_{i}^{K^{*}}\right)$ & $-1.52_{-0.34}^{+0.24} \pm 0.03$ & $9.10_{-1.46}^{+1.35} \pm 0.13$ \\
$T_{2}^{B_{s} \rightarrow K^{*}}$ & $B_{1}\left(1^{+}\right)$ & $0.21_{-0.03}^{+0.04}\left(\omega_{B_{s}}\right) \pm 0.003\left(a_{i}^{K^{*}}\right)$ & $-0.44_{-0.08}^{+0.13} \pm 0.01$ & $4.38_{-1.93}^{+1.33} \pm 0.16$ \\
$T_{3}^{B_{s} \rightarrow K^{*}}$ & $B_{1}\left(1^{+}\right)$ & $0.16_{-0.02}^{+0.03}\left(\omega_{B_{s}}\right) \pm 0.002\left(a_{i}^{K^{*}}\right)$ & $-0.99_{-0.27}^{+0.19} \pm 0.02$ & $8.12_{-1.81}^{+1.04} \pm 0.11$ \\
\hline \hline
\end{tabular}

shown in Table VII are the two major errors from the uncertainties of the parameter $\omega_{B_{s}}=0.50 \pm 0.05 \mathrm{GeV}$ and the Gegenbauer moments in the distribution amplitudes $a_{i}^{K}\left(a_{i}^{K^{*}}\right)$ of the light pseudoscalar (vector) mesons. The additional theoretical uncertainties from other input parameters, such as the decay constants $f_{B_{s}}, f_{K}, f_{K^{*}}, f_{K^{*}}^{T}$, are very small and have been neglected.

In Table VIII, we list the PQCD + Lattice predictions for the form factors $F_{+, 0}\left(q^{2}\right), V\left(q^{2}\right), A_{0,1,2}\left(q^{2}\right)$, and $T_{1,2,3}\left(q^{2}\right)$ by taking into account the lattice QCD results for the form factors at some points of $q^{2}$ as listed in Tables IV and V from Refs. $[82,83]$, in a similar way as what we did in Refs. [79-81]. The errors are obtained in the same way as those in Table VII. The additional form factors $A_{12}\left(q^{2}\right)$ and $T_{23}\left(q^{2}\right)$ can be defined as the linear combinations of $A_{1}\left(q^{2}\right)$ and $A_{2}\left(q^{2}\right), T_{2}\left(q^{2}\right)$ and $T_{3}\left(q^{2}\right)$, together the with kinematic variable $\lambda$ as given in Eqs. (10) and (11) from Ref. [83]. In Figs. 3 and 4, we show the $q^{2}$ dependence of the form factors $F_{+, 0, T}\left(q^{2}\right), V\left(q^{2}\right), A_{0,1,2}\left(q^{2}\right)$, and $T_{1,2,3}\left(q^{2}\right)$ in the PQCD (the red curves) and PQCD + Lattice (the blue curves) approaches for $B_{s} \rightarrow K^{(*)}$ transitions. The error bars of the initial PQCD and relevant lattice QCD results as listed in Tables IV and V are blackened, in order to show them clearly.

\section{B. Observables for $\boldsymbol{B}_{s} \rightarrow \mathrm{K} \boldsymbol{\ell}^{+} \boldsymbol{\ell}^{-}$}

From the differential decay rates as given in Eq. (58), it is conventional to make the integration over the range of $4 m_{\ell}^{2} \leq q^{2} \leq\left(m_{B_{s}}-m_{K}\right)^{2}$. In order to be consistent with the choices made by experiment collaborations in their data analysis, however, we have to cut off the regions of the dilepton mass squared around the charmonium resonances $J / \psi(1 S)$ and $\psi(2 S)$ : i.e., $8.0<q^{2}<11.0 \mathrm{GeV}^{2}$ and $12.5<q^{2}<15.0 \mathrm{GeV}^{2}$ for $\ell=(e, \mu, \tau)$ cases. The $\mathrm{PQCD}$ and PQCD + Lattice predictions for the BRs and the longitudinal polarization asymmetry $P_{L}$ of the semileptonic decays $\bar{B}_{s} \rightarrow K \ell^{+} \ell^{-}$and $B_{s} \rightarrow \bar{K} \ell^{+} \ell^{-}$at three different renormalization scales $\mu=\left(0.5 m_{b}, m_{b}, 1.5 m_{b}\right)$ are listed in Tables IX and X, respectively, where the total theoretical errors are the combinations of the uncertainties of all relevant input parameters: $\omega_{B_{s}}, a_{i}^{K}$ and $V_{i j}$. The direct $C P$ asymmetries $\mathcal{A}_{C P}$ are obtained by making integration

TABLE VIII. The PQCD + Lattice predictions for the form factors of $B_{s} \rightarrow K^{(*)}$ transitions. All form factors are fitted by using Eq. (98).

\begin{tabular}{lcccr}
\hline \hline PQCD + Lattice & $B\left(J^{P}\right)$ & $\alpha_{0}$ & \multicolumn{1}{c}{$\alpha_{1}$} & \multicolumn{1}{c}{$\alpha_{2}$} \\
\hline$F_{+}^{B_{s} \rightarrow K}$ & $B^{*}\left(1^{-}\right)$ & $0.22_{-0.06}^{+0.04}\left(\omega_{B_{s}}\right) \pm 0.005\left(a_{i}^{K}\right)$ & $-0.89_{-0.36}^{+0.23} \pm 0.02$ & $-0.44_{-0.44}^{+0.37} \pm 0.03$ \\
$F_{0}^{B_{s} \rightarrow K}$ & No pole & $0.22_{-0.06}^{+0.04}\left(\omega_{B_{s}}\right) \pm 0.005\left(a_{i}^{K}\right)$ & $-0.50_{-0.15}^{+0.08} \pm 0.01$ & $5.07_{-0.31}^{+0.27} \pm 0.02$ \\
$V^{B_{s} \rightarrow K^{*}}$ & $B^{*}\left(1^{-}\right)$ & $0.24_{-0.03}^{+0.05}\left(\omega_{B_{s}}\right) \pm 0.005\left(a_{i}^{K^{*}}\right)$ & $-0.13_{-0.07}^{+0.11} \pm 0.01$ & $10.14_{-0.49}^{+0.47} \pm 0.04$ \\
$A_{0}^{B_{s} \rightarrow K^{*}}$ & $B^{0}\left(0^{-}\right)$ & $0.21_{-0.03}^{+0.03}\left(\omega_{B_{s}}\right) \pm 0.003\left(a_{i}^{K^{*}}\right)$ & $-3.38_{-0.15}^{+0.17} \pm 0.02$ & $-4.13_{-0.49}^{+0.43} \pm 0.04$ \\
$A_{1}^{B_{s} \rightarrow K^{*}}$ & $B_{1}\left(1^{+}\right)$ & $0.19_{-0.03}^{+0.04}\left(\omega_{B_{s}}\right) \pm 0.004\left(a_{i}^{K^{*}}\right)$ & $-0.65_{-0.15}^{+0.27} \pm 0.02$ & $-2.66_{-0.32}^{+0.43} \pm 0.03$ \\
$A_{2}^{B_{s} \rightarrow K^{*}}$ & $B_{1}\left(1^{+}\right)$ & $0.19_{-0.03}^{+0.04}\left(\omega_{B_{s}}\right) \pm 0.004\left(a_{i}^{K^{*}}\right)$ & $-1.35_{-0.55}^{+0.58} \pm 0.04$ & $-3.12_{-1.43}^{+1.96} \pm 0.15$ \\
$T_{1}^{B_{s} \rightarrow K^{*}}$ & $B^{*}\left(1^{-}\right)$ & $0.21_{-0.03}^{+0.04}\left(\omega_{B_{s}}\right) \pm 0.003\left(a_{i}^{K^{*}}\right)$ & $-1.85_{-0.19}^{+0.30} \pm 0.02$ & $-3.23_{-1.32}^{+1.26} \pm 0.12$ \\
$T_{2}^{B_{s} \rightarrow K^{*}}$ & $B_{1}\left(1^{+}\right)$ & $0.21_{-0.03}^{+0.04}\left(\omega_{B_{s}}\right) \pm 0.003\left(a_{i}^{K^{*}}\right)$ & $-0.65_{-0.16}^{+0.29} \pm 0.02$ & $-2.85_{-0.55}^{+0.73} \pm 0.05$ \\
$T_{3}^{B_{s} \rightarrow K^{*}}$ & $B_{1}\left(1^{+}\right)$ & $0.16_{-0.02}^{+0.03}\left(\omega_{B_{s}}\right) \pm 0.002\left(a_{i}^{K^{*}}\right)$ & $-1.00_{-0.15}^{+0.19} \pm 0.02$ & $2.82_{-1.08}^{+1.26} \pm 0.09$ \\
\hline \hline
\end{tabular}



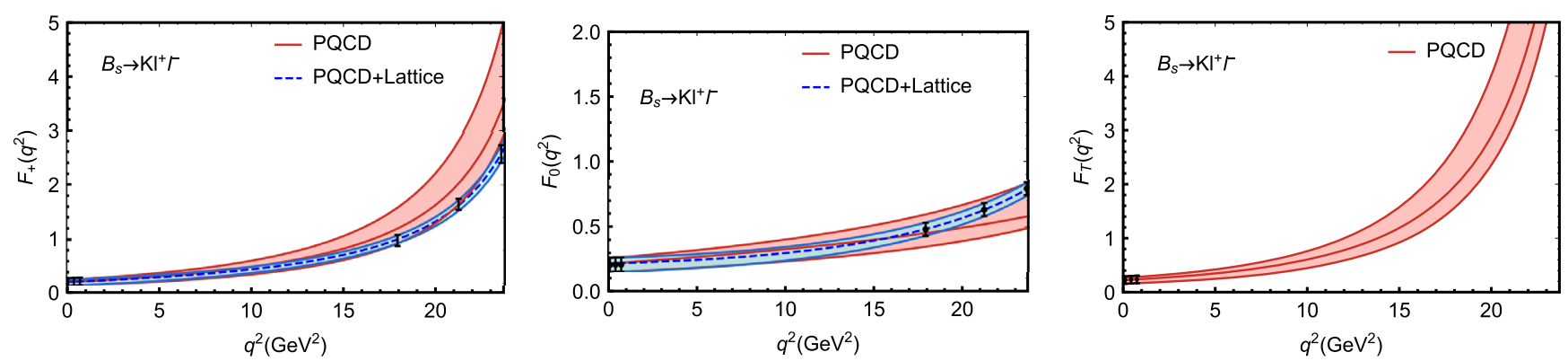

FIG. 3. The $q^{2}$ dependence of the form factors $F_{+, 0, T}\left(q^{2}\right)$ in the PQCD (red) and PQCD + Lattice (blue) approaches for $B_{s} \rightarrow K$ transition, while the red (blue) shaded band shows the major theoretical uncertainty.
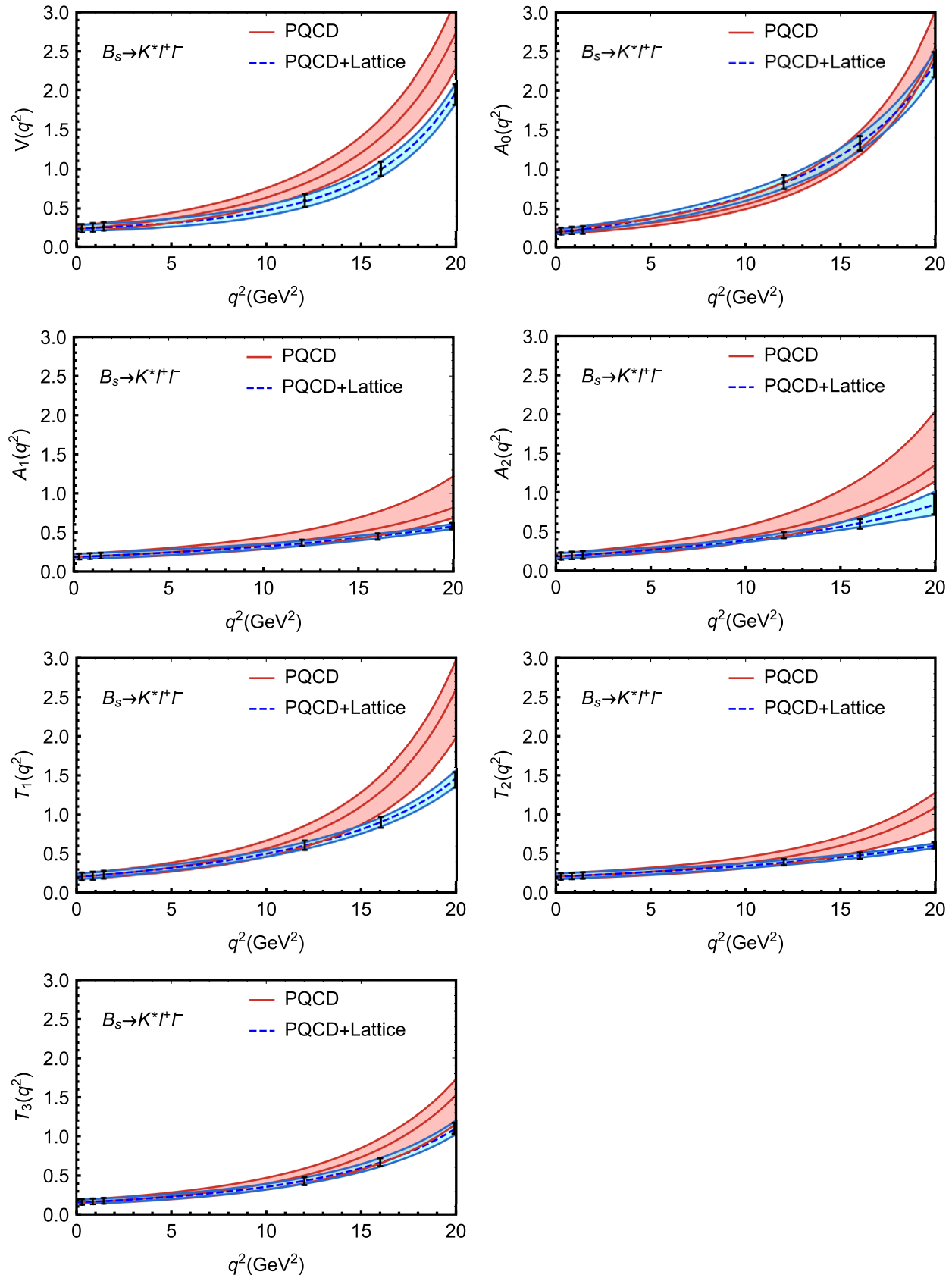

FIG. 4. The $q^{2}$ dependence of the form factors $V\left(q^{2}\right), A_{0,1,2}\left(q^{2}\right)$, and $T_{1,2,3}\left(q^{2}\right)$ in the PQCD (red) and PQCD + Lattice (blue) approaches for $B_{s} \rightarrow K^{*}$ transition, while the red (blue) shaded band shows the major theoretical uncertainty. 
TABLE IX. The PQCD predictions for the branching ratios (in units of $10^{-8}$ ), the longitudinal polarization asymmetry $P_{L}$ and the ratios $\left(R_{K}^{e \mu}, R_{K}^{\mu \tau}\right)$ of the decays $\bar{B}_{s} \rightarrow K \ell^{+} \ell^{-}$(the first row) and $B_{s} \rightarrow \bar{K} \ell^{+} \ell^{-}$(the second row) at three different renormalization scales. The direct $C P$ asymmetries $\mathcal{A}_{C P}$ (in units of $10^{-2}$ ) are also listed.

\begin{tabular}{|c|c|c|c|c|c|}
\hline Mode & Observables & $\mu=0.5 m_{b}$ & $\mu=m_{b}$ & $\mu=1.5 m_{b}$ & $\mathrm{PQCD}[26]$ \\
\hline \multirow[t]{5}{*}{$\ell=e$} & $\mathcal{B}\left(\bar{B}_{s} \rightarrow K \ell^{+} \ell^{-}\right)$ & $1.17_{-0.56}^{+0.68}$ & $1.24_{-0.60}^{+0.70}$ & $1.26_{-0.61}^{+0.71}$ & $1.63_{-0.58}^{+0.73}$ \\
\hline & $\mathcal{B}\left(B_{s} \rightarrow \bar{K} \ell^{+} \ell^{-}\right)$ & $1.21_{-0.59}^{+0.56}$ & $1.32_{-0.64}^{+0.73}$ & $1.63_{-0.66}^{+0.74}$ & $\ldots$ \\
\hline & $P_{L}\left(\bar{B}_{s} \rightarrow K \ell^{+} \ell^{-}\right)$ & $-0.986 \pm 0.002$ & $-0.979 \pm 0.002$ & $-0.972 \pm 0.002$ & $\ldots$ \\
\hline & $P_{L}\left(B_{s} \rightarrow \bar{K} \ell^{+} \ell^{-}\right)$ & $-0.980 \pm 0.002$ & $-0.954 \pm 0.008$ & $-0.937 \pm 0.004$ & $\cdots$ \\
\hline & $\mathcal{A}_{C P}$ & $-1.7 \pm 0.9$ & $-3.1 \pm 0.4$ & $-3.8 \pm 0.6$ & $\ldots$ \\
\hline \multirow[t]{5}{*}{$\ell=\mu$} & $\mathcal{B}$ & $1.17_{-0.56}^{+0.68}$ & $1.24_{-0.60}^{+0.71}$ & $1.25_{-0.61}^{+0.71}$ & $1.63_{-0.58}^{+0.73}$ \\
\hline & & $1.21_{-0.69}^{+0.60}$ & $1.32_{-0.64}^{+0.73}$ & $1.35_{-0.66}^{+0.74}$ & $\ldots$ \\
\hline & $P_{L}$ & $-0.974 \pm 0.002$ & $-0.966 \pm 0.002$ & $-0.959 \pm 0.002$ & $\ldots$ \\
\hline & & $-0.964 \pm 0.003$ & $-0.938 \pm 0.005$ & $-0.920 \pm 0.004$ & $\cdots$ \\
\hline & $\mathcal{A}_{C P}$ & $-1.7 \pm 0.9$ & $-3.1 \pm 0.4$ & $-3.8 \pm 0.6$ & $\cdots$ \\
\hline \multirow[t]{9}{*}{$\ell=\tau$} & $\mathcal{B}$ & $0.380_{-0.157}^{+0.202}$ & $0.382_{-0.158}^{+0.204}$ & $0.377_{-0.156}^{+0.203}$ & $0.43_{-0.15}^{+0.18}$ \\
\hline & & $0.382_{-0.158}^{+0.202}$ & $0.386_{-0.159}^{+0.206}$ & $0.382_{-0.158}^{+0.204}$ & $\cdots$ \\
\hline & $P_{L}$ & $-0.347 \pm 0.002$ & $-0.348 \pm 0.002$ & $-0.346 \pm 0.002$ & $\cdots$ \\
\hline & & $-0.348 \pm 0.002$ & $-0.349 \pm 0.002$ & $-0.348 \pm 0.001$ & $\ldots$ \\
\hline & $\mathcal{A}_{C P}$ & $-0.3 \pm 0.2$ & $-0.5 \pm 0.2$ & $-0.7 \pm 0.2$ & $\ldots$ \\
\hline & $R_{K}^{e \mu}$ & $0.996 \pm 0.002$ & $0.996 \pm 0.002$ & $0.996 \pm 0.002$ & 1 \\
\hline & & $0.996 \pm 0.002$ & $0.996 \pm 0.002$ & $0.996 \pm 0.002$ & $\ldots$ \\
\hline & $R_{K}^{\mu \tau}$ & $0.332 \pm 0.045$ & $0.325 \pm 0.045$ & $0.322 \pm 0.045$ & 0.26 \\
\hline & & $0.320 \pm 0.046$ & $0.303 \pm 0.046$ & $0.296 \pm 0.046$ & $\ldots$ \\
\hline
\end{tabular}

over $q^{2}$ for $\mathcal{A}_{C P}\left(q^{2}\right)$ as defined in Eqs. (62) and (81) and are also listed in these two tables. To reduce the large theoretical uncertainties, we also check the physical observables $R_{K}^{e \mu}$ and $R_{K}^{\mu \tau}$, as defined in Eqs. (3) and (4), i.e., the ratio of the branching ratios of $B_{s} \rightarrow K \ell^{+} \ell^{-}$ decays. As a comparison, the previous PQCD predictions as given in Ref. [26] for the decay rates and the ratios $R_{K}^{e \mu}$ and $R_{K}^{\mu \tau}$ are listed in last column of Table s IX and X.

TABLE X. The PQCD + Lattice predictions for the branching ratios (in unit of $10^{-8}$ ), the longitudinal polarization asymmetry $P_{L}$ and the direct $C P$ asymmetry $\mathcal{A}_{C P}$ (in unit of $10^{-2}$ ), as well as the ratios $\left(R_{K}^{e \mu}, R_{K}^{\mu \tau}\right)$ of the decays $\bar{B}_{s} \rightarrow K \ell^{+} \ell^{-}$(the first row) and $B_{s} \rightarrow \bar{K} \ell^{+} \ell^{-}$(the second row) at three different renormalization scales.

\begin{tabular}{|c|c|c|c|c|c|}
\hline Mode & Observables & $\mu=0.5 m_{b}$ & $\mu=m_{b}$ & $\mu=1.5 m_{b}$ & PQCD [26] \\
\hline$\ell=e$ & $\begin{aligned} & \mathcal{B}\left(\bar{B}_{s}\right.\left.\rightarrow K \ell^{+} \ell^{-}\right) \\
& \mathcal{B}\left(B_{s}\right.\left.\rightarrow \bar{K} \ell^{+} \ell^{-}\right) \\
& P_{L}\left(\bar{B}_{s}\right.\left.\rightarrow K \ell^{+} \ell^{-}\right) \\
& P_{L}\left(B_{s}\right.\left.\rightarrow \bar{K} \ell^{+} \ell^{-}\right) \\
& \mathcal{A}_{C P}\end{aligned}$ & $\begin{array}{c}0.95_{-0.31}^{+0.26} \\
0.99_{-0.33}^{+0.27} \\
-0.983 \pm 0.007 \\
-0.977 \pm 0.008 \\
-2.0 \pm 0.5\end{array}$ & $\begin{array}{c}1.01_{-0.33}^{+0.29} \\
1.10_{-0.38}^{+0.31} \\
-0.975 \pm 0.005 \\
-0.949 \pm 0.013 \\
-4.3 \pm 1.4\end{array}$ & $\begin{array}{c}1.03_{-0.35}^{+0.28} \\
1.14_{-0.40}^{+0.32} \\
-0.967 \pm 0.006 \\
-0.930 \pm 0.010 \\
-5.0 \pm 0.8\end{array}$ & $\begin{array}{c}1.63_{-0.58}^{+0.73} \\
\ldots \\
\ldots \\
\ldots \\
\ldots\end{array}$ \\
\hline$\ell=\mu$ & $\begin{array}{c}\mathcal{B} \\
P_{L} \\
\mathcal{A}_{C P}\end{array}$ & $\begin{array}{c}0.95_{-0.31}^{+0.25} \\
0.99_{-0.33}^{+0.27} \\
-0.968 \pm 0.008 \\
-0.958 \pm 0.009 \\
-2.0 \pm 0.5\end{array}$ & $\begin{array}{c}1.01_{-0.33}^{+0.27} \\
1.10_{-0.38}^{+0.31} \\
-0.960 \pm 0.007 \\
-0.929 \pm 0.012 \\
-4.3 \pm 1.4\end{array}$ & $\begin{array}{c}1.03_{-0.34}^{+0.28} \\
1.13_{-0.40}^{+0.32} \\
-0.953 \pm 0.008 \\
-0.911 \pm 0.014 \\
-5.1 \pm 0.8\end{array}$ & $\begin{array}{c}1.63_{-0.58}^{+0.73} \\
\ldots \\
\ldots \\
\ldots \\
\ldots\end{array}$ \\
\hline$\ell=\tau$ & $\begin{array}{l}P_{L} \\
\mathcal{A}_{C P} \\
R_{K}^{e \mu}\end{array}$ & $\begin{array}{c}0.365_{-0.075}^{+0.072} \\
0.366_{-0.075}^{+0.072} \\
-0.234 \pm 0.020 \\
-0.234 \pm 0.020 \\
-0.1 \pm 0.1 \\
0.996 \pm 0.002 \\
0.996 \pm 0.002 \\
0.395 \pm 0.081 \\
0.375 \pm 0.084\end{array}$ & $\begin{array}{c}0.368_{-0.076}^{+0.072} \\
0.370_{-0.076}^{+0.076} \\
-0.235 \pm 0.018 \\
-0.237 \pm 0.019 \\
-0.3 \pm 0.1 \\
0.996 \pm 0.002 \\
0.996 \pm 0.002 \\
0.384 \pm 0.080 \\
0.350 \pm 0.085\end{array}$ & $\begin{array}{c}0.365_{-0.075}^{+0.072} \\
0.368_{-0.076}^{+0.073} \\
-0.233 \pm 0.019 \\
-0.236 \pm 0.019 \\
-0.4 \pm 0.1 \\
0.996 \pm 0.002 \\
0.996 \pm 0.002 \\
0.381 \pm 0.080 \\
0.341 \pm 0.085\end{array}$ & $\begin{array}{c}0.43_{-0.15}^{+0.18} \\
\ldots \\
\ldots \\
\ldots \\
\ldots \\
1 \\
\ldots \\
0.26 \\
\ldots\end{array}$ \\
\hline
\end{tabular}


In order to show the major theoretical uncertainties from different sources explicitly, for instance, we show the PQCD predictions for $\mathcal{B}\left(\bar{B}_{s} \rightarrow K \mu^{+} \mu^{-}\right)$with the four kinds of errors:

$$
\begin{aligned}
\mathcal{B}\left(\bar{B}_{s} \rightarrow K \mu^{+} \mu^{-}\right)= & \left(1.24_{-0.58}^{+0.69}\left(\omega_{B_{s}}\right) \pm 0.15\left(a_{i}^{K}\right)\right. \\
& \left. \pm 0.06\left(V_{i j}\right)_{-0.07}^{+0.01}(\mu)\right) \times 10^{-8},
\end{aligned}
$$

where the dominant theoretical error comes from $\omega_{B_{s}}=$ $0.50 \pm 0.05$, the second one from the Gegenbauer moments $a_{1}^{K}=0.06 \pm 0.03$ and $a_{2}^{K}=0.25 \pm 0.15$ as given in Eq. (17), the third one from the CKM elements $V_{i j}$ in Eq. (96), and the last error from the renormalization scale $\mu=(1 \pm 0.5) m_{b}$. The possible errors from other input parameters are very small and have been neglected.

From Tables IX and X, it is easy to find the $C P$-averaged decay rates and the direct $C P$ asymmetries $\mathcal{A}_{C P}$ for the considered semileptonic decays:

$$
\begin{aligned}
\mathcal{B}\left(\bar{B}_{s}\right. & \left.\rightarrow K l^{+} l^{-}\right)\left.\right|_{\mathrm{CP}-\text { av }}, \\
= & \begin{cases}\left(1.28_{-0.48}^{+0.52}\right) \times 10^{-8}, & \text { PQCD }, \\
\left(1.06_{-0.29}^{+0.22}\right) \times 10^{-8}, & \mathrm{PQCD}+\text { Lattice, }\end{cases} \\
\mathcal{A}_{C P}\left(\bar{B}_{s} \rightarrow K l^{+} l^{-}\right) & \\
& = \begin{cases}-\left(3.1_{-1.5}^{+0.8}\right) \times 10^{-2}, & \text { PQCD }, \\
-\left(4.3_{-2.7}^{+1.6}\right) \times 10^{-2}, & \text { PQCD + Lattice, }\end{cases}
\end{aligned}
$$
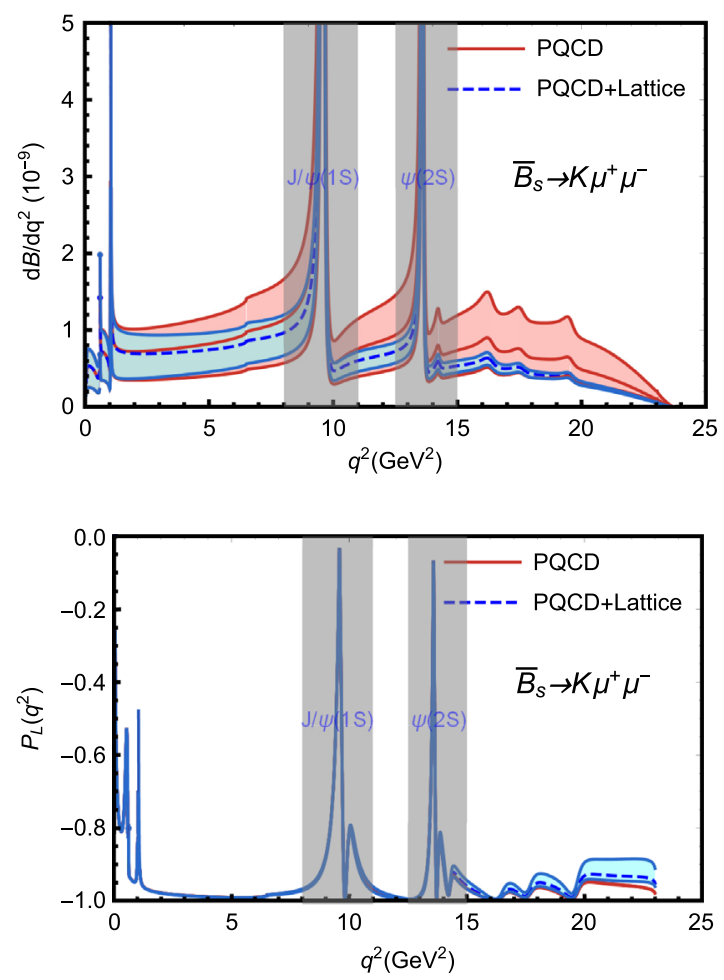

for the case of $l=(e, \mu)$, and

$$
\begin{gathered}
\left.\mathcal{B}\left(\bar{B}_{s} \rightarrow K \tau^{+} \tau^{-}\right)\right|_{\mathrm{CP}-\mathrm{av} .} \\
= \begin{cases}\left(0.38_{-0.12}^{+0.14}\right) \times 10^{-8}, & \mathrm{PQCD}, \\
\left(0.37_{-0.06}^{+0.05}\right) \times 10^{-8}, & \mathrm{PQCD}+\text { Lattice, }\end{cases} \\
\mathcal{A}_{C P}\left(\bar{B}_{s} \rightarrow K \tau^{+} \tau^{-}\right) \\
= \begin{cases}-(0.5 \pm 0.3) \times 10^{-2}, & \mathrm{PQCD}, \\
-(0.3 \pm 0.2) \times 10^{-2}, & \mathrm{PQCD}+\text { Lattice },\end{cases}
\end{gathered}
$$

for the case of $\tau$ lepton.

In Fig. 5, we show the $q^{2}$ dependence of the theoretical predictions of the differential branching fraction $d \mathcal{B} / d q^{2}$ and the longitudinal lepton polarization $P_{L}\left(q^{2}\right)$ for the decays $\bar{B}_{s} \rightarrow K \ell^{+} \ell^{-}$with $l=(\mu, \tau)$, evaluated by using the PQCD (the red solid curves) and the PQCD + Lattice (the blue dashed curves) approach, with the choice of the scale $\mu=m_{b}$ and $q_{\max }^{2}=23.71 \mathrm{GeV}^{2}$. The shaded bands indicate the theoretical error of our predictions due to the uncertainties of the input parameters. The two vertical grey blocks are the experimental veto regions [1] in order to remove contributions from $\bar{B}_{s} \rightarrow J / \psi(1 S)\left(\rightarrow \ell^{+} \ell^{-}\right) K$ (the left-hand band) and $\bar{B}_{s} \rightarrow \psi^{\prime}(2 S)\left(\rightarrow \ell^{+} \ell^{-}\right) K$ (the righthand band) for the $q^{2}$ dependence of $d \mathcal{B} / d q^{2}$ and $P_{L}$. The figure for the electron mode is almost identical with the one for muon, and therefore not shown here.
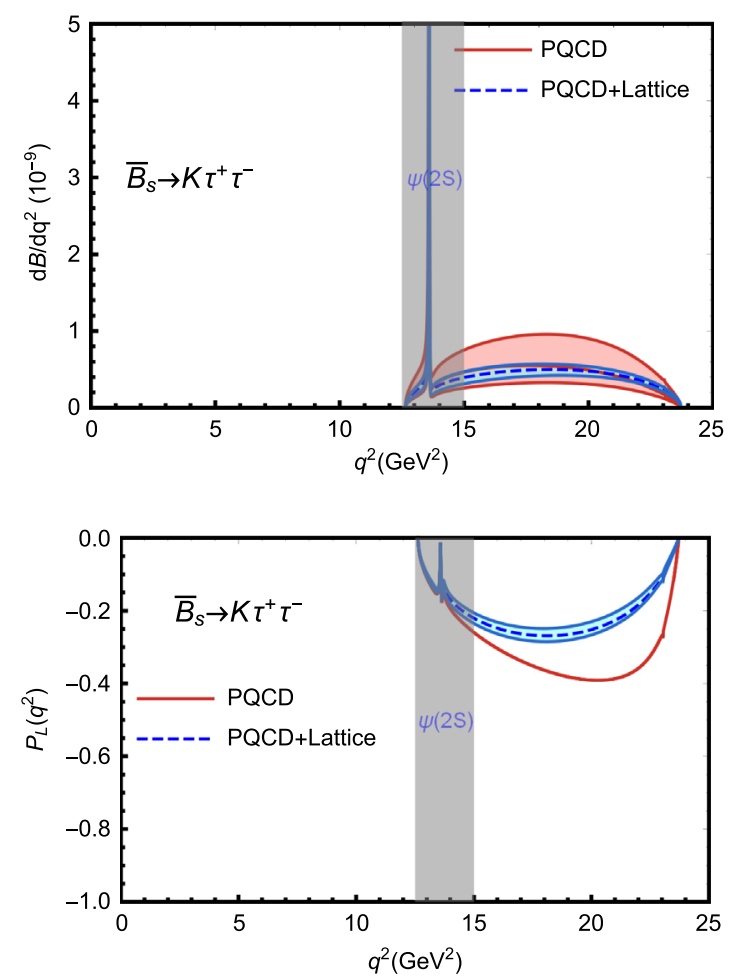

FIG. 5. The PQCD or "PQCD + Lattice" predictions for the $q^{2}$-dependence of $d \mathcal{B} / d q^{2}$ and $P_{L}\left(q^{2}\right)$ for the decays $\bar{B}_{s} \rightarrow K \ell^{+} \ell^{-}$with $l=\mu, \tau$. For details see the text. 
From the numerical results as listed in Tables IX and X, one can see the following points:

(1) The theoretical predictions from both PQCD and PQCD + Lattice approaches have a relatively weak dependence on the choice of the renormalization scale $\mu$. The variations of the central values due to the $\mu$ dependence are about $10 \%$ in magnitude and smaller than the combined errors from the uncertainties of other input parameters.

(2) Because of the term proportional to $\lambda_{u}$ in the effective Hamiltonian $\mathcal{H}_{\text {eff }}$ in Eq. (25), the PQCD and PQCD + Lattice predictions for the considered observables for $\bar{B}_{s} \rightarrow K l^{+} l^{-}$and its $C P$ conjugated mode have a relatively small differences, roughly $(5-15) \%$ in magnitude. For the $C P$-averaged branching ratios, the PQCD and PQCD + Lattice predictions agree well within 1 standard deviation. As generally expected, the direct $C P$ asymmetries $\mathcal{A}_{C P}$ are very small: less than $5 \%$.

(3) For the ratio $R_{K}^{e \mu}$, we find $R_{K}^{e \mu}=0.996(2)$ in both the PQCD and PQCD + Lattice approaches. For the ratio $R_{K}^{\mu \tau}$, the PQCD prediction is about 0.33 and a little smaller than the PQCD + Lattice prediction $R_{K}^{\mu \tau} \approx 0.39$, and they also show a weak $\mu$ dependence: less than $9 \%$ in magnitude for $0.5 m_{b} \leq$ $\mu \leq 1.5 m_{b}$.

(4) For the case of $l^{-}=\left(e^{-}, \mu^{-}\right)$, the PQCD and PQCD + Lattice predictions for the values of $P_{L}$ are very similar and close to -1 in value. For the $\tau^{-}$ lepton, however, the PQCD and PQCD + Lattice predictions for $P_{L}$ show a moderate difference: -0.35 against -0.24 .

\section{Observables for $\boldsymbol{B}_{s} \rightarrow \boldsymbol{K}^{*} \boldsymbol{\ell}^{+} \boldsymbol{\ell}^{-}$}

Analogous to the cases of $B_{s} \rightarrow K \ell^{+} \ell^{-}$decays, we follow the same procedure to calculate the physical observables of $B_{s} \rightarrow K^{*} \ell^{+} \ell^{-}$by using the PQCD and $\mathrm{PQCD}+$ Lattice approaches, respectively. For $B_{s} \rightarrow$ $K^{*} \ell^{+} \ell^{-}$decays, however, many more physical observables are defined and studied.

In Table XI we listed the PQCD and PQCD + Lattice predictions for the branching ratios $\mathcal{B}\left(\bar{B}_{s} \rightarrow K^{*} \ell^{+} \ell^{-}\right)$ and $\mathcal{B}\left(B_{s} \rightarrow \bar{K}^{*} \ell^{+} \ell^{-}\right)$with $l=(e, \mu, \tau)$, the lepton forward-backward asymmetries $\mathcal{A}_{F B}$, the longitudinal polarization asymmetries $F_{L}^{K^{*}}$ of the leptons, the direct $C P$ asymmetries $\mathcal{A}_{C P}$, and the ratios of the branching ratios $R_{K^{*}}^{e \mu}$ and $R_{K^{*}}^{\mu \tau}$ with the choice of the scale $\mu=m_{b}$. In numerical calculations, here we use the mean value of decay rate $\Gamma_{B_{s}^{0}}=1.509 \times 10^{12} \mathrm{~s}^{-1}$ [30]. For the branching ratios, the extra error from the $S$-wave pollution up to $10 \%$ should also be added [32]. In Table XII we listed the PQCD and PQCD + Lattice predictions for the values of those angular observables $P_{i}(i=1,2,3)$ and $P_{j}^{\prime}(j=4,5,6,8)$ in $l=(e, \mu, \tau)$ mode. The total errors of all theoretical predictions in Tables XI and XII are estimated in the same way as that for the case of $\bar{B}_{s} \rightarrow$ $K l^{+} l^{-}$decays.

From Table XI, it is easy to find the $C P$-averaged branching ratio $\mathcal{B}\left(\bar{B}_{s} \rightarrow K^{*} l^{+} l^{-}\right)$for $l=(e, \mu, \tau)$ :

TABLE XI. The PQCD and PQCD + Lattice predictions for the branching ratios (in unit of $10^{-8}$ ) of the semileptonic decays $B_{s} \rightarrow K^{*} \ell^{+} \ell^{-}$, the lepton forward-backward asymmetry $\mathcal{A}_{F B}$, the $K^{*}$ polarization fraction $F_{L}^{K^{*}}$, the direct $C P$ asymmetry $\mathcal{A}_{C P}$ (in unit of $10^{-2}$ ), and the ratios $R_{K^{*}}^{e \mu}$ and $R_{K^{*}}^{\mu \tau}$. The theoretical errors from the input parameters are combined in quadrature.

\begin{tabular}{|c|c|c|c|c|c|}
\hline \multirow[b]{2}{*}{ Mode } & \multirow[b]{2}{*}{ Observables } & \multicolumn{2}{|c|}{ PQCD } & \multicolumn{2}{|c|}{ PQCD + Lattice } \\
\hline & & $\overline{\bar{B}_{s}} \rightarrow K^{*} \ell^{+} \ell^{-}$ & $\overline{B_{s} \rightarrow \bar{K}^{*} \ell^{+} \ell^{-}}$ & $\overline{\bar{B}_{s}} \rightarrow K^{*} \ell^{+} \ell^{-}$ & $B_{s} \rightarrow \bar{K}^{*} \ell^{+} \ell^{-}$ \\
\hline \multirow[t]{2}{*}{$\ell=e$} & $\begin{array}{c}\mathcal{B} \\
\mathcal{A}_{F B} \\
F_{L}^{K^{*}}\end{array}$ & $\begin{array}{c}3.74_{-1.16}^{+1.38} \\
-0.244(9) \\
0.373(2)\end{array}$ & $\begin{array}{c}3.68_{-1.14}^{+1.33} \\
-0.235(17) \\
0.393(5)\end{array}$ & $\begin{array}{c}3.00_{-0.76}^{+0.91} \\
-0.183(10) \\
0.408(7)\end{array}$ & $\begin{array}{c}2.99_{-0.78}^{+0.94} \\
-0.176(10) \\
0.432(9)\end{array}$ \\
\hline & $\mathcal{A}_{C P}$ & \multicolumn{2}{|c|}{$0.8 \pm 0.3$} & \multicolumn{2}{|c|}{$0.2 \pm 0.4$} \\
\hline \multirow[t]{2}{*}{$\ell=\mu$} & $\begin{array}{c}\mathcal{B} \\
\mathcal{A}_{F B} \\
F_{L}^{K^{*}}\end{array}$ & $\begin{array}{c}3.20_{-1.00}^{+1.26} \\
-0.285(17) \\
0.434(12)\end{array}$ & $\begin{array}{c}3.14_{-0.98}^{+1.19} \\
-0.275(14) \\
0.457(16)\end{array}$ & $\begin{array}{c}2.48_{-0.59}^{+0.67} \\
-0.222(6) \\
0.494(16)\end{array}$ & $\begin{array}{c}2.47_{-0.61}^{+0.70} \\
-0.214(6) \\
0.522(18)\end{array}$ \\
\hline & $\mathcal{A}_{C P}$ & \multicolumn{2}{|c|}{$1.0 \pm 0.5$} & \multicolumn{2}{|c|}{$0.2 \pm 0.5$} \\
\hline \multirow[t]{2}{*}{$\ell=\tau$} & $\begin{array}{c}\mathcal{B} \\
\mathcal{A}_{F B} \\
F_{L}^{K^{*}}\end{array}$ & $\begin{array}{c}0.71_{-0.21}^{+0.35} \\
-0.235(33) \\
0.420(13)\end{array}$ & $\begin{array}{c}0.72_{-0.21}^{+0.36} \\
-0.232(33) \\
0.418(13)\end{array}$ & $\begin{array}{c}0.49 \pm 0.09 \\
-0.196(5) \\
0.480(5)\end{array}$ & $\begin{array}{c}0.50 \pm 0.09 \\
-0.194(5) \\
0.478(5)\end{array}$ \\
\hline & $\begin{array}{l}\mathcal{A}_{C P} \\
R_{K^{*}}^{e \mu} \\
R_{K^{*}}^{\mu \tau}\end{array}$ & $\begin{array}{c}0.993(2) \\
0.227(30)\end{array}$ & $\begin{array}{c}0.3 \\
0.993(2) \\
0.238(35)\end{array}$ & $\begin{array}{c}0.992(2) \\
0.205(20)\end{array}$ & $\begin{array}{c}0.2 \\
0.992(2) \\
0.211(22)\end{array}$ \\
\hline
\end{tabular}


TABLE XII. The PQCD and PQCD + Lattice predictions for the angular observables $P_{i}(i=1,2,3)$ and $P_{j}^{\prime}$ $(j=4,5,6,8)$ of the decays $\bar{B}_{s} \rightarrow K^{*} \ell^{+} \ell^{-}$(the first row) and $B_{s} \rightarrow \bar{K}^{*} \ell^{+} \ell^{-}$(the second row). The total uncertainties of the input parameters are combined in quadrature.

\begin{tabular}{|c|c|c|c|c|c|c|}
\hline \multirow[b]{2}{*}{ Obs. } & \multicolumn{2}{|c|}{$e$ Mode } & \multicolumn{2}{|c|}{$\mu$ Mode } & \multicolumn{2}{|c|}{$\tau$ Mode } \\
\hline & PQCD & PQCD + Lattice & PQCD & PQCD + Lattice & PQCD & PQCD + Lattice \\
\hline$-P_{1}$ & $\begin{array}{l}0.34 \pm 0.12 \\
0.33 \pm 0.11\end{array}$ & $\begin{array}{l}0.37 \pm 0.05 \\
0.36 \pm 0.05\end{array}$ & $\begin{array}{l}0.44 \pm 0.12 \\
0.44 \pm 0.12\end{array}$ & $\begin{array}{l}0.52 \pm 0.04 \\
0.52 \pm 0.04\end{array}$ & $\begin{array}{l}0.62 \pm 0.12 \\
0.62 \pm 0.12\end{array}$ & $\begin{array}{l}0.68 \pm 0.02 \\
0.68 \pm 0.02\end{array}$ \\
\hline$-P_{2}$ & $\begin{array}{l}0.27 \pm 0.01 \\
0.26 \pm 0.01\end{array}$ & $\begin{array}{l}0.21 \pm 0.01 \\
0.21 \pm 0.01\end{array}$ & $\begin{array}{l}0.34 \pm 0.03 \\
0.35 \pm 0.03\end{array}$ & $\begin{array}{l}0.30 \pm 0.01 \\
0.31 \pm 0.01\end{array}$ & $\begin{array}{l}0.36 \pm 0.05 \\
0.36 \pm 0.05\end{array}$ & $\begin{array}{l}0.35 \pm 0.01 \\
0.35 \pm 0.01\end{array}$ \\
\hline$-P_{3} \times 10^{3}$ & $\begin{array}{l}0.17 \pm 1.17 \\
1.27 \pm 0.99\end{array}$ & $\begin{array}{l}0.34 \pm 0.21 \\
2.84 \pm 0.51\end{array}$ & $\begin{array}{l}0.19 \pm 1.43 \\
1.68 \pm 1.15\end{array}$ & $\begin{array}{l}0.45 \pm 0.29 \\
4.11 \pm 0.52\end{array}$ & $\begin{array}{l}0.35 \pm 0.99 \\
0.38 \pm 1.08\end{array}$ & $\begin{array}{l}0.91 \pm 0.13 \\
0.98 \pm 0.14\end{array}$ \\
\hline$P_{4}^{\prime}$ & $\begin{array}{l}1.06 \pm 0.07 \\
1.00 \pm 0.01\end{array}$ & $\begin{array}{l}1.06 \pm 0.04 \\
0.99 \pm 0.04\end{array}$ & $\begin{array}{l}1.10 \pm 0.07 \\
1.07 \pm 0.07\end{array}$ & $\begin{array}{l}1.11 \pm 0.03 \\
1.07 \pm 0.03\end{array}$ & $\begin{array}{l}1.27 \pm 0.05 \\
1.27 \pm 0.05\end{array}$ & $\begin{array}{l}1.29 \pm 0.01 \\
1.29 \pm 0.01\end{array}$ \\
\hline$-P_{5}^{\prime}$ & $\begin{array}{l}0.57 \pm 0.06 \\
0.57 \pm 0.05\end{array}$ & $\begin{array}{l}0.48 \pm 0.02 \\
0.48 \pm 0.02\end{array}$ & $\begin{array}{l}0.61 \pm 0.07 \\
0.61 \pm 0.06\end{array}$ & $\begin{array}{l}0.52 \pm 0.02 \\
0.53 \pm 0.02\end{array}$ & $\begin{array}{l}0.58 \pm 0.10 \\
0.57 \pm 0.10\end{array}$ & $\begin{array}{l}0.54 \pm 0.01 \\
0.54 \pm 0.01\end{array}$ \\
\hline$-P_{6}^{\prime} \times 10^{3}$ & $\begin{array}{l}0.76 \pm 0.15 \\
0.77 \pm 0.14\end{array}$ & $\begin{array}{l}0.96 \pm 0.09 \\
0.96 \pm 0.08\end{array}$ & $\begin{array}{l}0.68 \pm 0.13 \\
0.70 \pm 0.13\end{array}$ & $\begin{array}{l}0.87 \pm 0.08 \\
0.87 \pm 0.07\end{array}$ & $\begin{array}{l}0.11 \pm 0.03 \\
0.11 \pm 0.02\end{array}$ & $\begin{array}{l}0.12 \pm 0.01 \\
0.12 \pm 0.01\end{array}$ \\
\hline$-P_{8}^{\prime} \times 10^{2}$ & $\begin{array}{l}0.61 \pm 0.06 \\
1.33 \pm 0.09\end{array}$ & $\begin{array}{l}0.67 \pm 0.07 \\
1.70 \pm 0.06\end{array}$ & $\begin{array}{l}-0.36 \pm 0.27 \\
2.20 \pm 0.27\end{array}$ & $\begin{array}{l}-0.57 \pm 0.26 \\
2.83 \pm 0.26\end{array}$ & $\begin{array}{l}0.27 \pm 0.07 \\
0.30 \pm 0.07\end{array}$ & $\begin{array}{l}0.37 \pm 0.03 \\
0.39 \pm 0.03\end{array}$ \\
\hline
\end{tabular}

$$
\begin{aligned}
\left.\mathcal{B}\left(\bar{B}_{s} \rightarrow K^{*} e^{+} e^{-}\right)\right|_{\text {CP-av. }} \\
\quad= \begin{cases}\left(3.71_{-0.82}^{+0.98} \pm 0.37\right) \times 10^{-8}, & \text { PQCD }, \\
\left(3.00_{-0.55}^{+0.66} \pm 0.30\right) \times 10^{-8}, & \text { PQCD + Lattice, }\end{cases}
\end{aligned}
$$

$$
\begin{aligned}
\left.\mathcal{B}\left(\bar{B}_{s} \rightarrow K^{*} \mu^{+} \mu^{-}\right)\right|_{\mathrm{CP}-\text { av. }} & \\
& = \begin{cases}\left(3.17_{-0.71}^{+0.89} \pm 0.32\right) \times 10^{-8}, & \mathrm{PQCD}, \\
\left(2.48_{-0.43}^{+0.50} \pm 0.25\right) \times 10^{-8}, & \mathrm{PQCD}+\text { Lattice },\end{cases}
\end{aligned}
$$

$$
\begin{aligned}
\left.\mathcal{B}\left(\bar{B}_{s} \rightarrow K^{*} \tau^{+} \tau^{-}\right)\right|_{\text {CP-av. }} \\
\quad= \begin{cases}\left(0.72_{-0.17}^{+0.25} \pm 0.07\right) \times 10^{-8}, & \text { PQCD }, \\
(0.50 \pm 0.06 \pm 0.05) \times 10^{-8}, & \text { PQCD + Lattice }\end{cases}
\end{aligned}
$$

where the second errors come from the $10 \% S$-wave pollution as estimated in Ref. [32].

In Fig. 6, we show the PQCD and the PQCD + Lattice predictions of $q^{2}$ dependence of the differential decay rate $d \mathcal{B} / d q^{2}$, the forward-backward asymmetry $\mathcal{A}_{F B}\left(q^{2}\right)$, the longitudinal polarization $F_{L}^{K^{*}}\left(q^{2}\right)$ for $B_{s} \rightarrow K^{*} \ell^{+} \ell^{-}$decays with $\ell=(\mu, \tau), q_{\max }^{2}=20.02 \mathrm{GeV}^{2}$, and the renormalization scale $\mu=m_{b}$. The red (blue) lines (dashed lines) correspond to the predictions obtained using the PQCD (PQCD + Lattice) approach, while the shaded narrow bands (red and blue) indicate the uncertainty of our predictions due to the variations of the input parameters.
For the cases of the decays $B_{s} \rightarrow K^{*} \mu^{+} \mu^{-}$, the two vertical grey blocks show the experimental veto regions [1] in order to remove the contributions from the resonance $J / \psi(1 S)$ (left-hand band) and $\psi^{\prime}(2 S)$ (right-hand band) to the form factor dependent observables. For the case of the decay $B_{s} \rightarrow K^{*} \tau^{+} \tau^{-}$, on the other hand, there is one vertical grey block which shows the experimental veto region [1] for the resonance $\psi^{\prime}(2 S)$ only.

In Figs. 7 and 8, we show the $q^{2}$ dependence of the angular observables $P_{i}(i=1,2,3)$ and $P_{j}^{\prime}(j=4,5,6,8)$ for the considered semileptonic decays $B_{s} \rightarrow K^{*} l^{+} l^{-}$with $\ell=(\mu, \tau)$, respectively. Since the relevant figures for the electron mode are very similar with those for the muon mode, we do not draw them in Figs. 6-8. The symbols in these two figures have the same meaning as those in Fig. 5.

From the numerical predictions as given in Tables XI and XII and in Figs. 6-8, we find the following points about the physical observables of the $\bar{B}_{s} \rightarrow K^{*} l^{+} l^{-}(\ell=e, \mu, \tau)$ decays:

(1) For the considered decay modes, the PQCD and $\mathrm{PQCD}+$ Lattice predictions for $\mathcal{B}\left(\bar{B}_{s} \rightarrow K^{*} l^{+} l^{-}\right)$ with $\ell=(e, \mu, \tau)$ do agree well with each other within the errors. The PQCD + Lattice predictions of $\mathcal{B}\left(\bar{B}_{s} \rightarrow K^{*} l^{+} l^{-}\right)$have smaller errors than those of the PQCD predictions. Both PQCD and PQCD + Lattice predictions of $\mathcal{B}\left(\bar{B}_{s} \rightarrow K^{*} \mu^{+} \mu^{-}\right)$do agree well with the LCSR prediction $(2.85 \pm 0.72) \times 10^{-8}$ [29] and with the currently available LHCb measured value $(2.9 \pm 1.1) \times 10^{-8}$ [20]. For the electron and muon modes, on the other hand, we have to wait for the future experimental measurements. 

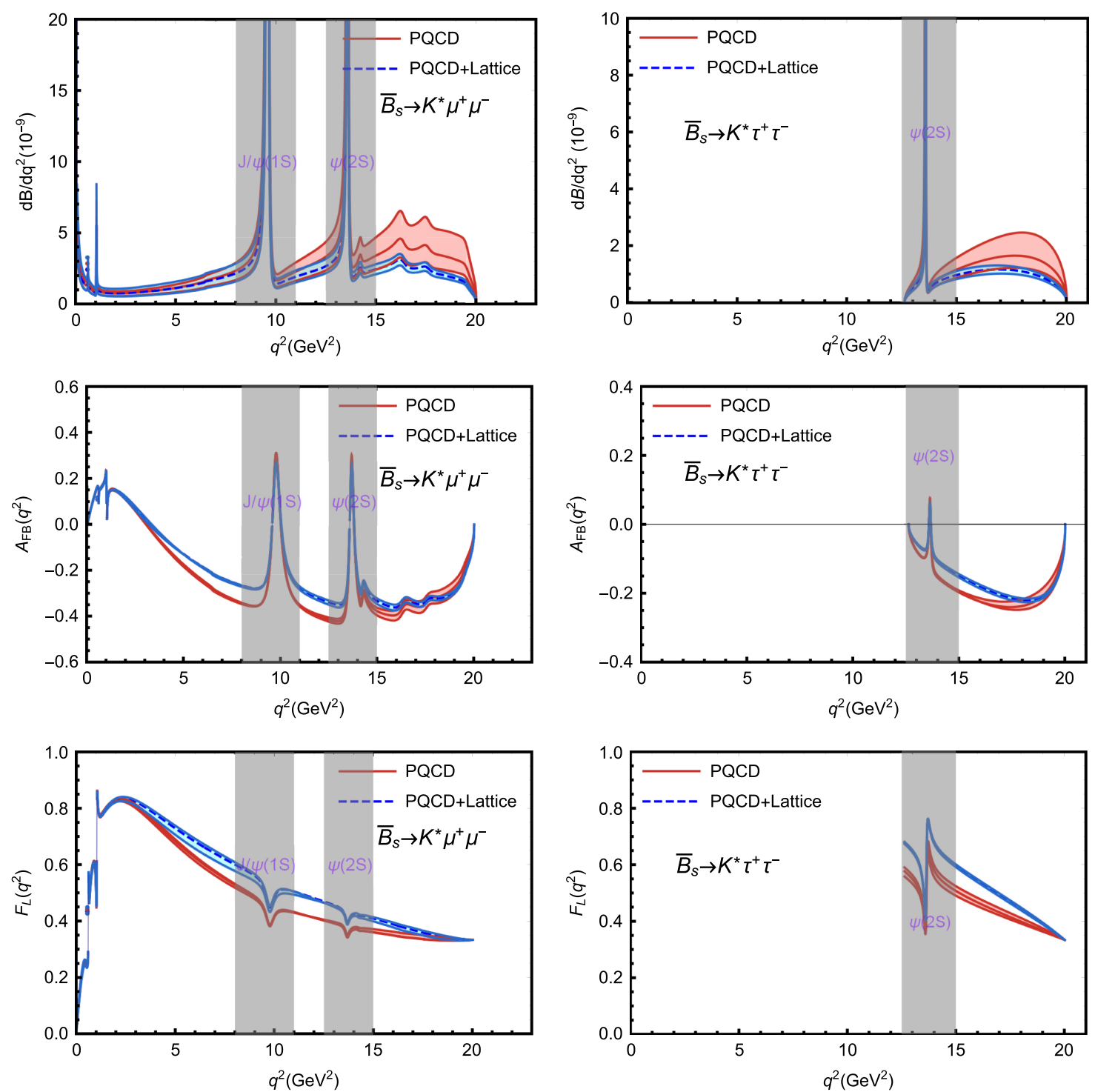

FIG. 6. The theoretical predictions for the $q^{2}$ dependence of $d \mathcal{B} / d q^{2}, \mathcal{A}_{F B}\left(q^{2}\right)$, and $F_{L}^{K^{*}}\left(q^{2}\right)$ for the decays $\bar{B}_{s} \rightarrow K^{*} \ell^{+} \ell^{-}$with $\ell=(\mu, \tau)$ in the PQCD and PQCD + Lattice approaches. For more details see the text.

(2) For the ratio $R_{K^{*}}^{e \mu}$, the theoretical predictions from both PQCD and PQCD + Lattice approaches are almost the same one, with a tiny $\sim 1 \%$ error because of the great cancellation of the errors in the ratio of the branching ratios. For the ratio $R_{K^{*}}^{\mu \tau}$, however, the remaining error of the theoretical predictions from both PQCD and PQCD + Lattice approaches are still around $10 \%$. These two ratios should be measured in the future experiments.

(3) For the direct $C P$ asymmetries $\mathcal{A}_{C P}$ of the considered decays, they are always very small as expected: less than $2 \%$ in magnitude. For physical observables $\mathcal{A}_{F B}$ and $F_{L}^{K^{*}}$, the differences between the central values of the PQCD and PQCD + Lattice are about $20 \%$ in magnitude, while the errors of the theoretical predictions are less than $10 \%$.
(4) For the angular observables $P_{i}$ and $P_{j}^{\prime}$, the PQCD and PQCD + Lattice predictions for each mode are consistent within errors. The values of $P_{3}$ and $P_{6,8}^{\prime}$ are close to zero: $\sim 10^{-2}$ for $P_{8}^{\prime}$ and $\sim 10^{-3}$ for $P_{3}$ and $P_{6}^{\prime}$. For the remaining $P_{1,2}$ and $P_{4,5}^{\prime}$, their magnitudes are small: $-1<\left(P_{1,2}, P_{5}^{\prime}\right)<-0.2$ while $P_{4}^{\prime} \sim 1$.

(5) One can see from the curves in Figs. 7 and 8 that most angular observables $P_{i}$ and $P_{j}^{\prime}$ have weak $q^{2}$ dependence in the major region of $q^{2}$ due to the large cancellation of $q^{2}$ dependence in the ratios.

For the semileptonic decays $\bar{B}_{s} \rightarrow K^{*} \ell^{+} \ell^{-}(\ell=e, \mu)$, some regions of $q^{2}$ do correspond to some resonance states, such as the charmonium $J / \Psi, \psi(2 S)$, etc., and should be removed for the sake of data analysis. Following Ref. [29], 

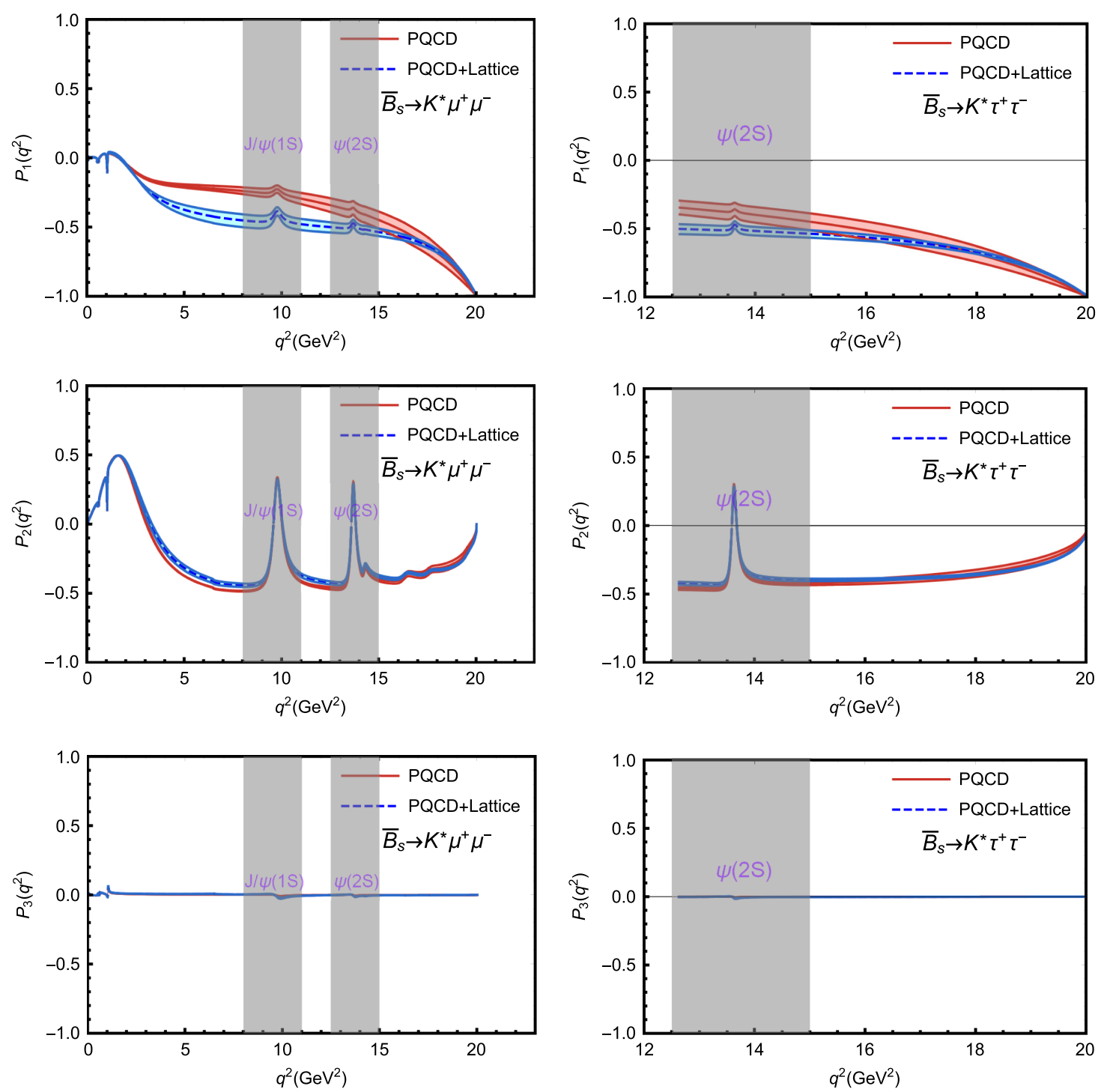

FIG. 7. The theoretical predictions for the $q^{2}$ dependence of the angular observables $P_{i}(i=1,2,3)$ for the decays $\bar{B}_{s} \rightarrow K^{*} l^{+} l^{-}$ $(\ell=\mu, \tau)$ in the PQCD and PQCD + Lattice approaches. For details see the text.

we here also present the binned value of the observables as a function of lepton-pair momentum $q^{2}$ covering two $q^{2}$ regions: [0.1-0.98] $\mathrm{GeV}^{2}$ and [1.1-6] $\mathrm{GeV}^{2}$ and consider the mass effect in the final state. We employ the PQCD and PQCD + Lattice approach to evaluate the form factors and compare the resultant results. Analogous to Ref. [29], we also define the $q^{2}$-binned observables in the following form:

$$
\begin{gathered}
\left\langle P_{1}\right\rangle_{b i n}=\frac{\int_{b i n} d q^{2}\left(I_{3}\right)}{2 \int_{b i n} d q^{2}\left(I_{2}^{s}\right)}, \quad\left\langle P_{2}\right\rangle_{b i n}=\frac{\int_{b i n} d q^{2}\left(\beta_{\ell} I_{6}^{s}\right)}{8 \int_{b i n} d q^{2}\left(I_{2}^{s}\right)}, \quad\left\langle P_{3}\right\rangle_{b i n}=-\frac{\int_{b i n} d q^{2}\left(I_{9}\right)}{4 \int_{b i n} d q^{2}\left(I_{2}^{s}\right)}, \\
\left\langle P_{4}^{\prime}\right\rangle_{b i n}=\frac{\int_{b i n} d q^{2}\left(I_{4}\right)}{\sqrt{-\int_{b i n} d q^{2}\left(I_{2}^{c} I_{2}^{s}\right)}}, \quad\left\langle P_{5}^{\prime}\right\rangle_{b i n}=\frac{\int_{b i n} d q^{2}\left(\beta_{\ell} I_{5}\right)}{2 \sqrt{-\left(\int_{b i n} d q^{2}\left(I_{2}^{c} I_{2}^{s}\right)\right)}}, \\
\left\langle P_{6}^{\prime}\right\rangle_{b i n}=-\frac{\int_{b i n} d q^{2}\left(\beta_{\ell} I_{7}\right)}{2 \sqrt{-\left(\int_{b i n} d q^{2}\left(I_{2}^{c} I_{2}^{s}\right)\right)}}, \quad\left\langle P_{8}^{\prime}\right\rangle_{b i n}=-\frac{\int_{b i n} d q^{2}\left(I_{8}\right)}{\sqrt{-\left(\int_{b i n} d q^{2}\left(I_{2}^{c} I_{2}^{s}\right)\right)}},
\end{gathered}
$$



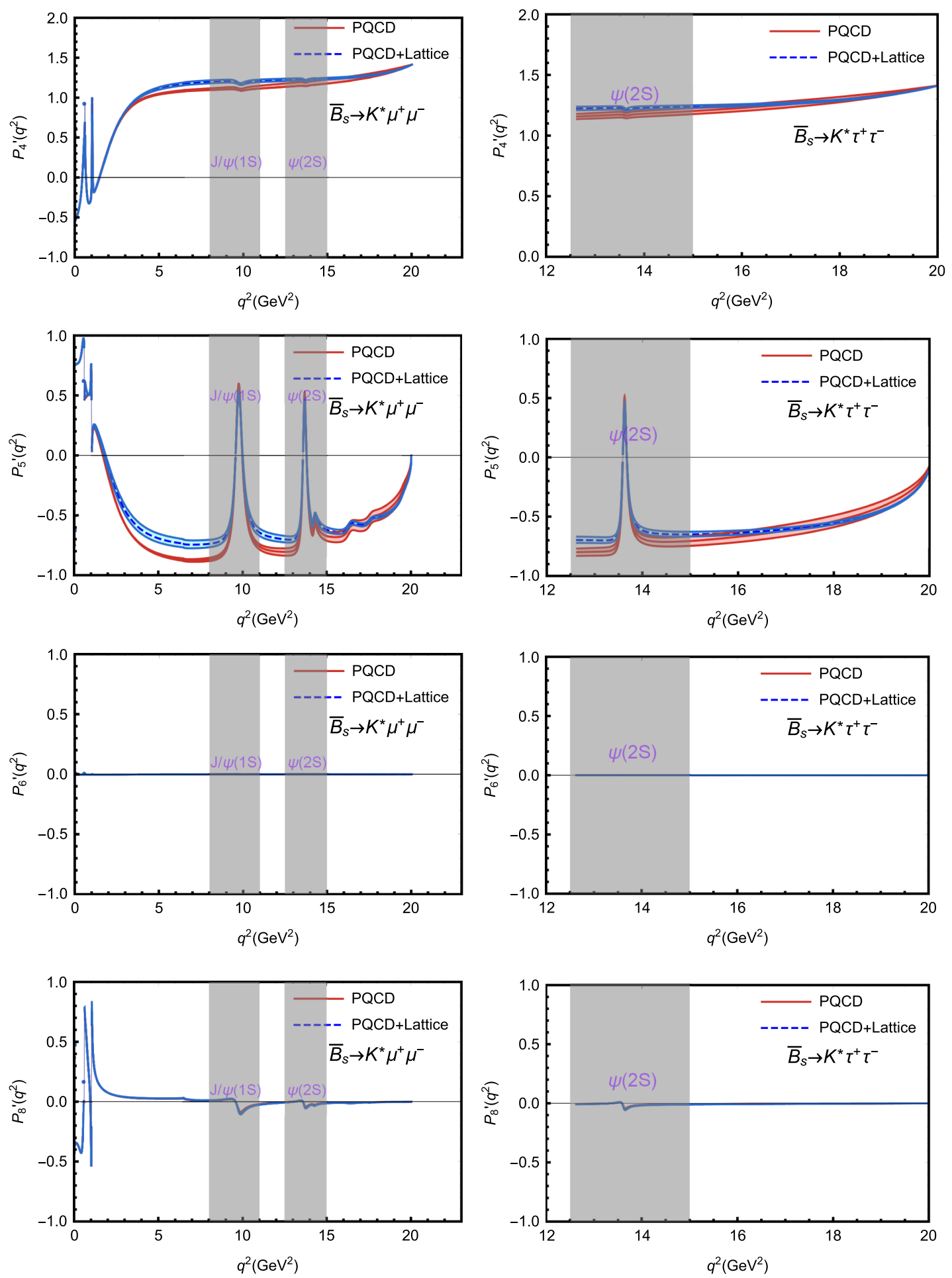

FIG. 8. The theoretical predictions for the $q^{2}$ dependence of the angular observables $P_{j}^{\prime}(j=4,5,6,8)$ for the decays $\bar{B}_{s} \rightarrow K^{*} l^{+} l^{-}$ $(\ell=\mu, \tau)$ in the PQCD and PQCD + Lattice approaches. For details see the text.

$$
\begin{gathered}
\langle\mathcal{B}\rangle_{\text {bin }}=\int_{\text {bin }} d q^{2} \frac{d \mathcal{B}\left(B_{s} \rightarrow K^{*} \ell^{+} \ell^{-}\right)}{d q^{2}}, \\
\left\langle R_{K^{*}}\right\rangle_{\text {bin }}=\frac{\langle B R\rangle_{\text {bin }}(\ell=\mu)}{\langle B R\rangle_{\text {bin }}(\ell=e)},
\end{gathered}
$$

$$
\begin{aligned}
& \left\langle\mathcal{A}_{\mathrm{FB}}(\ell)\right\rangle_{b i n}=\frac{\int_{b i n} d q^{2} b_{\theta_{\ell}}\left(q^{2}\right)}{\int_{b i n} d q^{2} d \Gamma / d q^{2}}=\frac{3 \int_{b i n} d q^{2} I_{6}^{s}}{4 \Gamma_{b i n}}, \\
& \left\langle F_{L}^{K^{*}}\right\rangle_{b i n}=\frac{3 \int_{b i n} d q^{2}\left(I_{1}^{c}-I_{2}^{c}\right)}{\int_{b i n} d q^{2}\left[3\left(I_{1}^{c}+2 I_{1}^{s}\right)-\left(I_{2}^{c}+2 I_{2}^{s}\right)\right]} .
\end{aligned}
$$


TABLE XIII. The binned values of observables for the process $\bar{B}_{s} \rightarrow K^{*} \mu^{+} \mu^{-}$and $B_{s} \rightarrow \bar{K}^{*} \mu^{+} \mu^{-}$at $\mu=m_{b}$ scale using the PQCD and PQCD + Lattice factorization approaches. The uncertainties shown are due to errors in determination of form factors and CKM parameters. The LCSR predictions for $B_{s} \rightarrow K^{*} \mu^{+} \mu^{-}$decay as given in Ref. [29] were added as a comparison. For details see the text.

\begin{tabular}{|c|c|c|c|c|c|}
\hline \multirow{2}{*}{\multicolumn{2}{|c|}{ Decay Mode Observables/Bin }} & \multicolumn{2}{|c|}{$\bar{B}_{s} \rightarrow K^{*} \mu^{+} \mu^{-}$} & \multicolumn{2}{|c|}{$B_{s} \rightarrow \bar{K}^{*} \mu^{+} \mu^{-}$} \\
\hline & & {$[0.1-0.98] \mathrm{GeV}^{2}$} & {$[1.1-6] \mathrm{GeV}^{2}$} & {$[0.1-0.98] \mathrm{GeV}^{2}$} & {$[1.1-6] \mathrm{GeV}^{2}$} \\
\hline$\left\langle P_{1}\right\rangle$ & $\begin{array}{l}\text { PQCD } \\
\text { PQCD + Lattice } \\
\text { LCSR [29] }\end{array}$ & $\begin{array}{c}0.004(2) \\
0.003(2) \\
0.012(129)\end{array}$ & $\begin{array}{c}-0.157(16) \\
-0.279(25) \\
-0.081(111)\end{array}$ & $\begin{array}{c}0.023(2) \\
0.021(3) \\
0.011(135)\end{array}$ & $\begin{array}{c}-0.163(16) \\
-0.292(28) \\
-0.075(108)\end{array}$ \\
\hline$\left\langle P_{2}\right\rangle$ & $\begin{array}{l}\text { PQCD } \\
\text { PQCD + Lattice } \\
\text { LCSR [29] }\end{array}$ & $\begin{array}{c}0.127(2) \\
0.128(2) \\
0.118(13)\end{array}$ & $\begin{array}{c}-0.127(6) \\
-0.101(18) \\
0.112(80)\end{array}$ & $\begin{array}{c}0.139(2) \\
0.141(2) \\
0.112(13)\end{array}$ & $\begin{array}{c}-0.223(5) \\
-0.155(16) \\
0.142(79)\end{array}$ \\
\hline$\left\langle P_{3}\right\rangle \times 10^{2}$ & $\begin{array}{l}\text { PQCD } \\
\text { PQCD + Lattice } \\
\text { LCSR [29] }\end{array}$ & $\begin{array}{l}0.1 \pm 0.1 \\
0.1 \pm 0.1 \\
0.1 \pm 0.2\end{array}$ & $\begin{array}{l}0.4 \pm 0.2 \\
0.8 \pm 0.2 \\
0.4 \pm 1.0\end{array}$ & $\begin{array}{l}0.2 \pm 0.2 \\
0.2 \pm 0.2 \\
0.1 \pm 0.7\end{array}$ & $\begin{array}{l}0.5 \pm 0.2 \\
0.9 \pm 0.2 \\
0.3 \pm 1.0\end{array}$ \\
\hline$\left\langle P_{4}^{\prime}\right\rangle$ & $\begin{array}{l}\text { PQCD } \\
\text { PQCD + Lattice } \\
\text { LCSR [29] }\end{array}$ & $\begin{array}{c}-0.131(2) \\
-0.131(3) \\
-0.593(58)\end{array}$ & $\begin{array}{c}0.817(14) \\
0.854(7) \\
0.464(165)\end{array}$ & $\begin{array}{l}-0.603(2) \\
-0.604(3) \\
-0.650(60)\end{array}$ & $\begin{array}{c}0.849(13) \\
0.890(9) \\
0.379(172)\end{array}$ \\
\hline$\left\langle P_{5}^{\prime}\right\rangle$ & $\begin{array}{l}\text { PQCD } \\
\text { PQCD + Lattice } \\
\text { LCSR [29] }\end{array}$ & $\begin{array}{c}0.711(3) \\
0.715(2) \\
0.547(53)\end{array}$ & $\begin{array}{c}-0.608(3) \\
-0.486(40) \\
-0.286(133)\end{array}$ & $\begin{array}{c}0.394(2) \\
0.392(2) \\
0.543(55)\end{array}$ & $\begin{array}{c}-0.650(4) \\
-0.524(39) \\
-0.273(140)\end{array}$ \\
\hline$\left\langle P_{6}^{\prime}\right\rangle \times 10^{2}$ & $\begin{array}{l}\text { PQCD } \\
\text { PQCD + Lattice } \\
\text { LCSR [29] }\end{array}$ & $\begin{array}{c}-0.4 \pm 0.2 \\
-0.3 \pm 0.5 \\
-10.4 \pm 1.7\end{array}$ & $\begin{array}{l}-0.2 \pm 0.2 \\
-0.2 \pm 0.2 \\
-9.5 \pm 1.1\end{array}$ & $\begin{array}{l}-0.3 \pm 0.2 \\
-0.3 \pm 0.2 \\
-6.9 \pm 0.5\end{array}$ & $\begin{array}{l}-0.2 \pm 0.2 \\
-0.2 \pm 0.2 \\
-7.8 \pm 0.4\end{array}$ \\
\hline$\left\langle P_{8}^{\prime}\right\rangle$ & $\begin{array}{l}\text { PQCD } \\
\text { PQCD + Lattice } \\
\text { LCSR [29] }\end{array}$ & $\begin{array}{c}0.042(2) \\
0.041(2) \\
0.015(16)\end{array}$ & $\begin{array}{c}0.050(2) \\
0.053(2) \\
0.040(17)\end{array}$ & $\begin{array}{c}0.044(2) \\
0.045(2) \\
0.044(16)\end{array}$ & $\begin{array}{c}0.057(2) \\
0.062(2) \\
0.034(19)\end{array}$ \\
\hline$\langle\mathcal{B}\rangle \times 10^{9}$ & $\begin{array}{l}\text { PQCD } \\
\text { PQCD + Lattice } \\
\text { LCSR [29] }\end{array}$ & $\begin{array}{c}1.44 \pm 0.47 \\
1.43_{-0.42}^{+0.48} \\
3.81 \pm 0.46\end{array}$ & $\begin{array}{c}4.65_{-1.39}^{+1.52} \\
4.64_{-1.41}^{+1.54} \\
7.80 \pm 1.79\end{array}$ & $\begin{array}{c}1.72_{-0.49}^{+0.69} \\
1.71_{-0.48}^{+0.66} \\
4.41 \pm 0.57\end{array}$ & $\begin{array}{c}4.99_{-1.65}^{+2.09} \\
4.98_{-1.71}^{+2.13} \\
8.39 \pm 1.89\end{array}$ \\
\hline$\left\langle R_{K^{*}}\right\rangle$ & $\begin{array}{l}\text { PQCD } \\
\text { PQCD + Lattice } \\
\text { LCSR [29] }\end{array}$ & $\begin{array}{l}0.983(1) \\
0.982(1) \\
0.940(9)\end{array}$ & $\begin{array}{l}0.995(1) \\
0.996(1) \\
0.998(4)\end{array}$ & $\begin{array}{l}0.984(1) \\
0.984(1) \\
0.942(8)\end{array}$ & $\begin{array}{l}0.996(1) \\
0.997(1) \\
0.998(4)\end{array}$ \\
\hline$\left\langle A_{F B}(\ell)\right\rangle$ & $\begin{array}{l}\text { PQCD } \\
\text { PQCD + Lattice } \\
\text { LCSR [29] }\end{array}$ & $\begin{array}{c}0.110(2) \\
0.110(2) \\
-0.060(8)\end{array}$ & $\begin{array}{l}-0.067(5) \\
-0.034(4) \\
-0.029(22)\end{array}$ & $\begin{array}{c}0.076(2) \\
0.077(2) \\
-0.056(8)\end{array}$ & $\begin{array}{c}-0.087(5) \\
-0.053(3) \\
-0.036(22)\end{array}$ \\
\hline$\left\langle F_{L}^{K^{*}}\right\rangle$ & $\begin{array}{l}\text { PQCD } \\
\text { PQCD + Lattice } \\
\text { LCSR [29] }\end{array}$ & $\begin{array}{l}0.297(5) \\
0.297(10) \\
0.453(68)\end{array}$ & $\begin{array}{l}0.741(11) \\
0.769(16) \\
0.853(39)\end{array}$ & $\begin{array}{l}0.543(8) \\
0.543(13) \\
0.464(65)\end{array}$ & $\begin{array}{l}0.738(10) \\
0.769(16) \\
0.851(39)\end{array}$ \\
\hline
\end{tabular}

In Table XIII, we listed the PQCD and PQCD + Lattice predictions for the binned values of all eleven physical observables considered in this paper for the $\bar{B}_{s} \rightarrow K^{*} \mu^{+} \mu^{-}$and the $B_{s} \rightarrow \bar{K}^{*} \mu^{+} \mu^{-}$decays. The theoretical errors from the input parameters are combined in quadrature in the tabulated error estimates. As a comparison, we also insert an extra row of the results from the LCSR approach [29] into the table, for each physical observable. It is necessary to note that there exist three differences between our predictions and the LCSR results as given in Ref. [29]:
(1) The sign definition of the forward-backward asymmetry $A_{F B}$ in Ref. [29] is opposite to ours as given in Eq. (76).

(2) Our choices of the $q^{2}$ bin are $[0.1-0.98] \mathrm{GeV}^{2}$ and [1.1-6] GeV $\mathrm{Ge}^{2}$, instead of the $[0.1-1] \mathrm{GeV}^{2}$ and [1-6] $\mathrm{GeV}^{2}$ in Ref. [29], because we try to remove the possible contribution from the light resonance $\phi(1020)$.

(3) The authors in Ref. [29] considered the nonfactorizable corrections like weak annihilation and spectator scattering in the bin $[1-6] \mathrm{GeV}^{2}$ while these 
effects in our analysis are very small and have been neglected.

On the theoretical side, from the numerical results as listed in Table XIII, one can find the following points:

(1) For binned values of observables $\left\langle P_{1,2}\right\rangle$ and $\left\langle A_{F B}(\ell)\right\rangle$, the differences between the PQCD and $\mathrm{PQCD}+$ Lattice predictions are around (20-40)\% of the central values. For other eight physical observables, however, the PQCD and PQCD + Lattice predictions agree very well within errors. The source of the difference comes from a little different $q^{2}$ dependence of the form factors of these two factorization approaches.

(2) The differences between our results and LCSR predictions [29] are generally not large in magnitude and could be understood if one takes the three differences between our approaches and the LCSR as specified in the previous paragraph. Current differences will be tested in the future when the experimental measurements become available.

(3) For observables $\left\langle P_{3}\right\rangle$ and $\left\langle P_{6,8}^{\prime}\right\rangle$, their $\mathrm{SM}$ values are tiny, about $10^{-3}$ to $10^{-2}$ in magnitude, because they are basically driven by the NLO contributions. It is noted that the observable $P_{6}^{\prime}$ stems from the absorptive part of $b \rightarrow d \gamma$, a small imaginary number. Since these observables are not protected from hadronic uncertainties in general, their values are more sensitive to the choice of the method of calculating the form factors or to the variations of the input parameters being used in calculations.

(4) In this paper, the possible long-distance charm-loop effects have been taken into account. The modification induced to $C_{9}$ is encoded in a shift where the factorizable charm loop and nonfactorizable soft gluon are taken into account. We also use a phenomenological model to account for light resonances like $\rho(770)$ and $\omega(782)$ in the low $-q^{2}$ region. It is interesting to note that such a particular effect is difficult to estimate and can be large in size, casting some doubts on the possibility to exploit the bins between $J / \psi$ and $\psi(2 S)$ for comparison with experiments.

\section{SUMMARY AND CONCLUSIONS}

In the framework of the SM, here we studied the rare semileptonic decays $\bar{B}_{s} \rightarrow K^{(*)} \ell^{+} \ell^{-}$with $l^{-}=$ $\left(e^{-}, \mu^{-}, \tau^{-}\right)$by using the PQCD and PQCD + Lattice factorization approaches and provided the theoretical predictions for the thirteen kinds of physical observables: the branching ratios $\mathcal{B}\left(\bar{B}_{s} \rightarrow K^{(*)} \ell^{+} \ell^{-}\right), \mathcal{B}\left(B_{s} \rightarrow \bar{K}^{(*)} \ell^{+} \ell^{-}\right)$ and their $C P$ averages, the ratios of the branching ratios $R_{K, K^{*}}^{e \mu}$ and $R_{K, K^{*}}^{\mu \tau}$, the lepton FB asymmetry $\mathcal{A}_{F B}(l)$, the longitudinal polarization asymmetry of the leptons $P_{L}$ and the quantity $F_{L}^{K^{*}}$, the angular observables $P_{i}$ with $(i=1,2,3)$, and $P_{j}^{\prime}$ with $(j=4,5,6,8)$. In the PQCD factorization approach, specifically, we first evaluated the relevant form factors $F_{0,+, T}\left(q^{2}\right), V\left(q^{2}\right), A_{0,1,2}\left(q^{2}\right)$ and $T_{1,2,3}\left(q^{2}\right)$ in the low- $q^{2}$ region and then extrapolate them to the whole $q^{2}$ region using the BCL parametrization method. In the PQCD + Lattice approach, we also take those currently available lattice QCD results for the relevant form factors at the end point $q_{\max }^{2}$ as additional input to improve the extrapolation of the form factors from the low $-q^{2}$ region to the whole range of $q^{2}$.

Based on our numerical calculations and the phenomenological analysis, we find the following main points:

(1) For all physical observables considered in this paper, the PQCD and PQCD + Lattice predictions do agree well within 1 standard deviation. The theoretical errors of the PQCD + Lattice predictions for the branching ratios become much smaller than those of the PQCD predictions.

(2) For $\bar{B}_{s} \rightarrow\left(K, K^{*}\right) \mu^{+} \mu^{-}$decays, for example, the PQCD and PQCD + Lattice predictions for the $C P$ averaged branching ratios are the following:

$$
\begin{aligned}
&\left.\mathcal{B}\left(\bar{B}_{s} \rightarrow K \mu^{+} \mu^{-}\right)\right|_{\mathrm{CP}-\mathrm{av} .} \\
&= \begin{cases}\left(1.28_{-0.48}^{+0.52}\right) \times 10^{-8}, & \text { PQCD, } \\
\left(1.06_{-0.29}^{+0.22}\right) \times 10^{-8}, & \text { PQCD + Lattice, }\end{cases} \\
&\left.\mathcal{B}\left(\bar{B}_{s} \rightarrow K^{*} \mu^{+} \mu^{-}\right)\right|_{\mathrm{CP} \text {-av. }} \\
&= \begin{cases}\left(3.17_{-0.78}^{+0.95}\right) \times 10^{-8}, & \text { PQCD }, \\
\left(2.48_{-0.50}^{+0.56}\right) \times 10^{-8}, & \text { PQCD + Lattice. }\end{cases}
\end{aligned}
$$

Our theoretical predictions for the $\mathcal{B}\left(\bar{B}_{s} \rightarrow K^{*} \mu^{+} \mu^{-}\right)$ do agree well with the measured one $(2.9 \pm 1.1) \times$ $10^{-8}$ as reported by the LHCb Collaboration [20].

(3) For the ratios $R_{K^{*}}^{e \mu}$ and $R_{K^{*}}^{\mu \tau}$, the PQCD and PQCD + Lattice predictions agree very well and have a small error less than $10 \%$ due to the cancellation of the theoretical uncertainties in the ratios of the branching ratios. For the direct $C P$ asymmetries $\mathcal{A}_{C P}$, they are always very small: less than $5 \%$ in magnitude. For physical observables $\mathcal{A}_{F B}$ and $F_{L}^{K^{*}}$, the differences between the central values of the PQCD and PQCD + Lattice are about $(10 \sim 30) \%$ in magnitude, while the errors of the theoretical predictions are less than $10 \%$.

(4) For the angular observables $P_{1,2,3}$ and $P_{4,5,6,8}^{\prime}$, the PQCD and PQCD + Lattice predictions for each lepton $l^{-}$are consistent within errors. The theoretical predictions of $P_{3}$ and $P_{6.8}^{\prime}$ are tiny, say less than $10^{-2}$ in absolute value, and thus hardly to be measured. For the remaining $P_{1,2}$ and $P_{4,5}^{\prime}$, on the other hand, 
their magnitudes are larger than 0.2 and therefore could be measured by future LHCb and Belle-II experiments.

(5) For the sake of data analysis, we also defined eleven $q^{2}$-binned observables and presented our theoretical predictions of the binned values of all considered observables with the choice of two $q^{2}$ bins $[0.1-0.98] \mathrm{GeV}^{2}$ and $[1.1-6] \mathrm{GeV}^{2}$. The PQCD and PQCD + Lattice predictions generally agree with each other and are also consistent with most LCSR results within errors.

In general, we believe that most physical observables considered in this paper could be measured in the future $\mathrm{LHCb}$ or Belle-II experiments. Any clear deviations from above SM predictions might be a signal of new physics beyond the SM.

\section{ACKNOWLEDGMENTS}

This work was supported by the National Natural Science Foundation of China under Grants No. 11775117 and No. 11235005.

\section{APPENDIX: RELEVANT FUNCTIONS}

The threshold resummation factor $S_{t}(x)$ is adopted from [76]:

$$
S_{t}=\frac{2^{1+2 c} \Gamma(3 / 2+c)}{\sqrt{\pi} \Gamma(1+c)}[x(1-x)]^{c},
$$

and here we set the parameter $c=0.3$. The hard functions $h_{1}$ and $h_{2}$ come form the Fourier transform and can be written as

$$
\begin{aligned}
h_{1}\left(x_{1}, x_{2}, b_{1}, b_{2}\right)= & K_{0}\left(\beta_{1} b_{1}\right)\left[\theta\left(b_{1}-b_{2}\right) I_{0}\left(\alpha_{1} b_{2}\right) K_{0}\left(\alpha_{1} b_{1}\right)\right. \\
& \left.+\theta\left(b_{2}-b_{1}\right) I_{0}\left(\alpha_{1} b_{1}\right) K_{0}\left(\alpha_{1} b_{2}\right)\right] S_{t}\left(x_{2}\right),
\end{aligned}
$$

$$
\begin{aligned}
h_{2}\left(x_{1}, x_{2}, b_{1}, b_{2}\right)= & K_{0}\left(\beta_{2} b_{1}\right)\left[\theta\left(b_{1}-b_{2}\right) I_{0}\left(\alpha_{2} b_{2}\right) K_{0}\left(\alpha_{2} b_{1}\right)\right. \\
& \left.+\theta\left(b_{2}-b_{1}\right) I_{0}\left(\alpha_{2} b_{1}\right) K_{0}\left(\alpha_{2} b_{2}\right)\right] S_{t}\left(x_{2}\right),
\end{aligned}
$$

where $K_{0}$ and $I_{0}$ are modified Bessel functions, and

$$
\begin{aligned}
& \alpha_{1}=m_{B_{s}} \sqrt{x_{2} r \eta^{+}}, \quad \alpha_{2}=m_{B_{s}} \sqrt{x_{1} r \eta^{+}-r^{2}+r_{d}^{2}}, \\
& \beta_{1}=\beta_{2}=m_{B_{s}} \sqrt{x_{1} x_{2} r \eta^{+}},
\end{aligned}
$$

where $r=m_{K^{(*)}} / m_{B_{s}}, r_{d}=m_{d} / m_{B_{s}}$.

The factor $\exp \left[-S_{a b}(t)\right]$ contains the Sudakov logarithmic corrections and the renormalization group evolution effects of both the wave functions and the hard scattering amplitude with $S_{a b}(t)=S_{B}(t)+S_{M}(t)$ [76],

$$
\begin{aligned}
S_{B}(t) & =s\left(x_{1} \frac{m_{B_{s}}}{\sqrt{2}}, b_{1}\right)+\frac{5}{3} \int_{1 / b_{1}}^{t} \frac{d \bar{\mu}}{\bar{\mu}} \gamma_{q}\left(\alpha_{s}(\bar{\mu})\right), \\
S_{M}(t)= & s\left(x_{2} \frac{m_{B_{s}}}{\sqrt{2}} r \eta^{+}, b_{2}\right)+s\left(\left(1-x_{2}\right) \frac{m_{B_{s}}}{\sqrt{2}} r \eta^{+}, b_{2}\right) \\
& +2 \int_{1 / b_{2}}^{t} \frac{d \bar{\mu}}{\bar{\mu}} \gamma_{q}\left(\alpha_{s}(\bar{\mu})\right),
\end{aligned}
$$

with the quark anomalous dimension $\gamma_{q}=-\alpha_{s} / \pi$. The explicit expressions of the functions $s(Q, b)$ can be found, for example, in Appendix A of Ref. [34]. The hard scales $t_{i}$ in the above equation are chosen as the largest scale of the virtuality of the internal particles in the hard $b$-quark decay diagrams,

$t_{1}=\max \left\{\alpha_{1}, 1 / b_{1}, 1 / b_{2}\right\}, \quad t_{2}=\max \left\{\alpha_{2}, 1 / b_{1}, 1 / b_{2}\right\}$.
[1] J.-T. Wei et al. (Belle Collaboration), Measurement of the Differential Branching Fraction and Forward-Backword Asymmetry for $B \rightarrow K^{(*)} \ell^{+} \ell^{-}$, Phys. Rev. Lett. 103, 171801 (2009).

[2] A. Ali, P. Ball, L. T. Handoko, and G. Hiller, A comparative study of the decays $B \rightarrow\left(K, K^{*}\right) \ell^{+} \ell^{-}$in standard model and supersymmetric theories, Phys. Rev. D 61, 074024 (2000).

[3] M. Beneke, T. Feldmann, and D. Seidel, Systematic approach to exclusive $B \rightarrow V \ell^{+} \ell^{-}, V \gamma$ decays, Nucl. Phys. B612, 25 (2001).

[4] C.H. Chen and C.Q. Geng, Exclusive decays of $B \rightarrow$ $K^{(*)} \ell^{+} \ell^{-}$in the PQCD, Phys. Rev. D 63, 114025 (2001).
[5] A. Ali, G. Kramer, and G. h. Zhu, $B \rightarrow K^{+} \ell^{+} \ell^{-}$decay in soft-collinear effective theory, Eur. Phys. J. C 47, 625 (2006).

[6] U. Egede, T. Hurth, J. Matias, M. Ramon, and W. Reece, New observables in the decay mode $\bar{B}_{d} \rightarrow \bar{K}^{* 0} \ell^{+} \ell^{-}$, J. High Energy Phys. 11 (2008) 032.

[7] C. Bobeth, G. Hiller, and G. Piranishvili, $C P$ asymmetries in bar $B \rightarrow \bar{K}^{*}(\rightarrow \bar{K} \pi) \bar{\ell} \ell$ and untagged $\bar{B}_{s}, \quad B_{s} \rightarrow \phi(\rightarrow$ $\left.K^{+} K^{-}\right) \bar{\ell} \ell$ decays at NLO, J. High Energy Phys. 07 (2008) 106.

[8] W. Altmannshofer, P. Ball, A. Bharucha, A. J. Buras, D. M. Straub, and M. Wick, Symmetries and asymmetries 
of $B \rightarrow K^{*} \mu^{+} \mu^{-}$decays in the Standard Model and beyond, J. High Energy Phys. 01 (2009) 019.

[9] T. Aaltonen et al. (CDF Collaboration), Measurement of the Forward-Backward Asymmetry in the $B \rightarrow K^{(*)} \mu^{+} \mu^{-}$ Decay and First Observation of the $B_{s}^{0} \rightarrow \phi \mu^{+} \mu^{-}$Decay, Phys. Rev. Lett. 106, 161801 (2011).

[10] J. P. Lees et al. (BABAR Collaboration), Measurement of branching fractions and rate asymmetries in the rare decays $B \rightarrow K^{(*)} l^{+} l^{-}$, Phys. Rev. D 86, 032012 (2012).

[11] R. Aaij et al. (LHCb Collaboration), Test of lepton universality using $B^{+} \rightarrow K^{+} \ell^{+} \ell^{-}$decays, Phys. Rev. Lett. 113, 151601 (2014).

[12] V. Khachatryan et al. (CMS Collaboration), Angular analysis of the decay $B^{0} \rightarrow K^{* 0} \mu^{+} \mu^{-}$from pp collisions at $\sqrt{s}=8 \mathrm{TeV}$, Phys. Lett. B 753, 424 (2016).

[13] R. Aaij et al. (LHCb Collaboration), Measurements of the S-wave fraction in $B^{0} \rightarrow K^{+} \pi^{-} \mu^{+} \mu^{-}$decays and the $B^{0} \rightarrow$ $K^{*}(892)^{0} \mu^{+} \mu^{-}$differential branching fraction, J. High Energy Phys. 11 (2016) 047.

[14] R. Aaij et al. (LHCb Collaboration), Test of lepton universality with $B^{0} \rightarrow K^{* 0} \ell^{+} \ell^{-}$decays, J. High Energy Phys. 08 (2017) 055.

[15] R. Aaij et al. (LHCb Collaboration), Measurement of Form-Factor-Independent Observables in the Decay $B^{0} \rightarrow$ $K^{* 0} \mu^{+} \mu^{-}$, Phys. Rev. Lett. 111, 191801 (2013).

[16] R. Aaij et al. (LHCb Collaboration), Angular analysis of the $B^{0} \rightarrow K^{* 0} \mu^{+} \mu^{-}$decay using $3 \mathrm{fb}^{-1}$ of integrated luminosity, J. High Energy Phys. 02 (2016) 104.

[17] S. L. Glashow, J. Iliopoulos, and L. Maiani, Weak interactions with Lepton-Hadron symmetry, Phys. Rev. D 2, 1285 (1970).

[18] M. Ciuchini, A. M. Coutinho, M. Fedele, E. Franco, A. Paul, L. Silvestrini, and M. Valli, On flavourful Easter eggs for new physics hunger and Lepton flavour universality violation, Eur. Phys. J. C 77, 688 (2017).

[19] R. Aaij et al. (LHCb Collaboration), First measurement of the differential branching fraction and $C P$ asymmetry of the $B^{ \pm} \rightarrow \pi^{ \pm} \mu^{+} \mu^{-}$decay, J. High Energy Phys. 10 (2015) 034.

[20] R. Aaij et al. (LHCb Collaboration), Evidence for the decay $B_{s}^{0} \rightarrow \bar{K}^{* 0} \mu^{+} \mu^{-}$, J. High Energy Phys. 07 (2018) 020.

[21] J. J. Wang, R. M. Wang, Y. G. Xu, and Y. D. Yang, The rare decays $B_{u}^{+} \rightarrow \pi^{+} l^{+} l^{-}, \rho^{+} l^{+} l^{-}$and $B_{d}^{0} \rightarrow l^{+} l^{-}$in the R-parity violating supersymmetry, Phys. Rev. D 77, 014017 (2008).

[22] A. Ali, A. Ya. Parkhomenko, and A. V. Rusov, Precise calculation of the dilepton invariant-mass spectrum and the decay rate in $B^{ \pm} \rightarrow \pi^{ \pm} \mu^{+} \mu^{-}$in the SM, Phys. Rev. D 89, 094021 (2014).

[23] W. S. Hou, M. Kohda, and F. Xu, Rates and asymmetries of $B \rightarrow \pi l^{+} l^{-}$decays, Phys. Rev. D 90, 013002 (2014).

[24] C. Hambrock, A. Khodjamirian, and A. Rusov, Hadronic effects and observables in $B \rightarrow \pi l^{+} l^{-}$decay at large recoil, Phys. Rev. D 92, 074020 (2015).

[25] J. A. Bailey et al. (Fermilab Lattice and MILC Collaborations), $B \rightarrow \pi l^{+} l^{-}$form Factors for New-Physics Searches from Lattice QCD, Phys. Rev. Lett. 115, 152002 (2015).

[26] W.F. Wang and Z.J. Xiao, The semileptonic decays $B / B_{s} \rightarrow(\pi, K)\left(\ell^{+} \ell^{-}, \ell \nu, \nu \bar{\nu}\right)$ in the perturbative QCD approach beyond the leading-order, Phys. Rev. D 86, 114025 (2012).
[27] W. F. Wang, Y. Y. Fan, M. Liu, and Z. J. Xiao, Semileptonic decays $B / B_{s} \rightarrow\left(\eta, \eta^{\prime}, G\right)\left(\ell^{+} \ell^{-}, \ell \bar{\nu}, \nu \bar{\nu}\right.$ in the perturbative QCD approach beyond the leading order, Phys. Rev. D 87, 097501 (2013).

[28] A. Khodjamirian and A. V. Rusov, $B_{s} \rightarrow K \ell \nu_{\ell}$ and $B_{(s)} \rightarrow$ $\pi(K) \ell^{+} \ell^{-}$decays at large recoil and CKM matrix elements, J. High Energy Phys. 08 (2017) 112.

[29] B. Kindra and N. Mahajan, Predictions of angular observables for $\bar{B}_{s} \rightarrow K^{*} \ell \ell$ and $\bar{B} \rightarrow \rho \ell \ell$ in the standard model, Phys. Rev. D 98, 094012 (2018).

[30] M. Tanabashi et al. (Particle Data Group), Review of particle physics, Phys. Rev. D 98, 030001 (2018).

[31] S. Aoki et al. (Flavour Lattice Averaging Group), FLAG Review 2019, Eur. Phys. J. C 80, 113 (2020).

[32] M. Döring, U. G. Meißner, and W. Wang, Chiral dynamics and S-wave contributions in semileptonic B decays, J. High Energy Phys. 10 (2013) 011.

[33] Y. Y. Kim, H. N. Li, and A. I. Sanda, Penguin enhancement and $B \rightarrow K \pi$ decays in perturbative QCD, Phys. Rev. D 63, 054008 (2001).

[34] C. D. Lu, K. Ukai, and M. Z. Yang, Branching ratio and $C P$ violation of $B \rightarrow \pi \pi$ decays in the perturbative QCD approach, Phys. Rev. D 63, 074009 (2001).

[35] H. N. Li, QCD Aspects of exclusive B meson decays, Prog. Part. Nucl. Phys. 51, 85 (2003), and references therein.

[36] C. Bourrely, I. Caprini, and L. Lellouch, Model-independent description of $B \rightarrow \pi l \nu$ decays and a determination of $\left|V_{u b}\right|$, Phys. Rev. D 79, 013008 (2009); Erratum, Phys. Rev. D 82, 099902 (2010).

[37] D. Leljak, B. Melic, and M. Patra, On lepton flavour universality in semileptonic $B_{c} \rightarrow \eta_{c}, J / \Psi$ decays, J. High Energy Phys. 05 (2019) 094.

[38] Y. Y. Fan, W. F. Wang, S. Cheng, and Z. J. Xiao, Semileptonic decays $B \rightarrow D^{(*)} l \nu$ in the perturbative QCD factorization approach, Chin. Sci. Bull. 59, 125 (2014).

[39] Z. J. Xiao, W. F. Wang, and Y. Y. Fan, Revisiting the pure annihilation decays $B_{s} \rightarrow \pi^{+} \pi^{-}$and $B^{0} \rightarrow K^{+} K^{-}$: The data and the pQCD predictions, Phys. Rev. D 85, 094003 (2012).

[40] Y. Y. Fan, W. F. Wang, S. Cheng, and Z. J. Xiao, Anatomy of $B \rightarrow K \eta^{\left({ }^{\prime}\right)}$ decays in different mixing schemes and effects of next-to-leading order contributions in the perturbative QCD approach, Phys. Rev. D 87, 094003 (2013).

[41] D. C. Yan, P. Yang, X. Liu, and Z. J. Xiao, Anatomy of $B_{s} \rightarrow P V$ decays and effects of next-to-leading order contributions in the perturbative QCD factorization approach, Nucl. Phys. B931, 79 (2018).

[42] D. C. Yan, X. Liu, and Z. J. Xiao, Anatomy of $B_{s} \rightarrow V V$ decays and effects of next-to-leading order contributions in the perturbative QCD factorization approach, Nucl. Phys. B935, 17 (2018).

[43] P. Ball, V. M. Braun, and A. Lenz, Higher-twist distribution amplitudes of the K meson in QCD, J. High Energy Phys. 05 (2006) 004.

[44] A. Ali, G. Kramer, Y. Li, C. D. Lu, Y. L. Shen, W. Wang, and Y. M. Wang, Charmless non-leptonic $B_{s}$ decays to $P P$, $P V$ and $V V$ final states in the pQCD approach, Phys. Rev. D 76, 074018 (2007).

[45] R. H. Li, C. D. Lu, W. Wang, and X.X. Wang, $B \rightarrow S$ transition form factors in the PQCD approach, Phys. Rev. D 79, 014013 (2009). 
[46] S. R. Singh and B. Mawlong, 331- $Z^{\prime}$ mediated FCNC effects on $b \rightarrow d \mu^{+} \mu^{-}$transitions, Int. J. Mod. Phys. A 33, 1850225 (2018).

[47] P. Nayek, P. Maji, and S. Sahoo, Study of semileptonic decays $B \rightarrow \pi \ell^{+} \ell^{-}$and $B \rightarrow \rho \ell^{+} \ell^{-}$in nonuniversal Z model, Phys. Rev. D 99, 013005 (2019).

[48] R. Khosravi, Form factors and branching ratios of the FCNC $B \rightarrow a_{1} \ell^{+} \ell^{-}$decays, Eur. Phys. J. C 75, 220 (2015).

[49] C.H. Chen and C.Q. Geng, Baryonic rare decays of $\Lambda(b) \rightarrow \Lambda \ell^{+} \ell^{-}$, Phys. Rev. D 64, 074001 (2001).

[50] G. Buchalla, A. J. Buras, and M. E. Lautenbacher, Weak decays beyond leading logarithms, Rev. Mod. Phys. 68, 1125 (1996).

[51] C. S. Lim, T. Morozumi, and A. I. Sanda, A prediction for $d \Gamma(b \rightarrow s \ell \bar{\ell}) / d q^{2}$ including the long distance effects, Phys. Lett. B 218, 343 (1989).

[52] N. G. Deshpande, J. Trampetic, and K. Panose, Resonance background to the decays $b \rightarrow s \ell^{+} \ell^{-}, B \rightarrow K^{*} \ell^{+} \ell^{-}$and $B \rightarrow K \ell^{+} \ell^{-}$, Phys. Rev. D 39, 1461 (1989).

[53] A. Ali, T. Mannel, and T. Morozumi, Forward backward asymmetry of dilepton angular distribution in the decay $b \rightarrow s \ell^{+} \ell^{-}$, Phys. Lett. B 273, 505 (1991).

[54] P. J. O'Donnell and H. K. K. Tung, Resonance contributions to the decay $b \rightarrow s \ell^{+} \ell^{-}$, Phys. Rev. D 43, R2067 (1991).

[55] M. Jezabek and J. H. Kuhn, Lepton spectra from heavy quark decay, Nucl. Phys. B320, 20 (1989).

[56] B. Grinstein, M. J. Savage, and M. B. Wise, $B \rightarrow X_{s} e^{+} e^{-}$in the six quark model, Nucl. Phys. B319, 271 (1989).

[57] M. Misiak, The $b \rightarrow s e^{+} e^{-}$and $b \rightarrow s \gamma$ decays with nextto-leading logarithmic QCD corrections, Nucl. Phys. B393, 23 (1993); B439, 461(E) (1995).

[58] A. J. Buras and M. Munz, Effective Hamiltonian for $B \rightarrow$ $X_{s} e^{+} e^{-}$beyond leading logarithms in the NDR and HV schemes, Phys. Rev. D 52, 186 (1995).

[59] A. Khodjamirian, T. Mannel, A. A. Pivovarov, and Y.-M. Wang, Charm-loop effect in $B \rightarrow K^{(*)} \ell^{+} \ell^{-}$and $B \rightarrow K^{*} \gamma$, J. High Energy Phys. 09 (2010) 089.

[60] D. P. Du, A. X. El-Khadra, S. Gottlieb, A. S. Kronfeld, J. Laiho, E. Lunghi, R. S. Van de Water, and R. Zhou (Fermilab Lattice and MILC Collaborations), Phenomenology of semileptonic B-meson decays with form factors from lattice QCD, Phys. Rev. D 93, 034005 (2016).

[61] R. Aaij et al. (LHCb Collaboration), Observation of a Resonance in $B^{+} \rightarrow K^{+} \mu^{+} \mu^{-}$Decays at Low Recoil, Phys. Rev. Lett. 111, 112003 (2013).

[62] C. D. Lu and D. X. Zhang, Reexamination of long distance effects in $b \rightarrow s \ell^{+} \ell^{-}$, Phys. Lett. B 397, 279 (1997).

[63] C. Bobeth, G. Hiller, D. van Dyk, and C. Wacker, The decay $B \rightarrow K \ell^{+} \ell^{-}$at low Hadronic recoil and model-independent $\Delta B=1$ constraints, J. High Energy Phys. 01 (2012) 107.

[64] C. Bobeth, G. Hiller, and G. Piranishvili, Angular distributions of $\bar{B} \rightarrow \bar{K} \ell^{+} \ell^{-}$decays, J. High Energy Phys. 12 (2007) 040.

[65] S. Descotes-Genon, T. Hurth, J. Matias, and J. Virto, Optimizing the basis of $B \rightarrow K^{*} l l$ observables in the full kinematic range, J. High Energy Phys. 05 (2013) 137.

[66] S. Descotes-Genon, J. Matias, M. Ramon, and J. Virto, Implications from clean observables for the binned analysis of $B \rightarrow K^{*} \mu^{+} \mu^{-}$at large recoil, J. High Energy Phys. 01 (2013) 048.

[67] D. Becirevic and E. Schneider, On transverse asymmetries in $B \rightarrow K^{*} l^{+} l^{-}$, Nucl. Phys. B854, 321 (2012).

[68] J. Matias, F. Mescia, M. Ramon, and J. Virto, Complete anatomy of $\bar{B}_{d} \rightarrow \bar{K}^{* 0}(\rightarrow K \pi) \ell^{+} \ell^{-}$and its angular distribution, J. High Energy Phys. 04 (2012) 104.

[69] D. Becirevic, M. Fedele, I. Nisandzic, and A. Tayduganov, Lepton flavor universality tests through angular observables of $\bar{B} \rightarrow D^{(*)} \ell \bar{\nu}$ decay modes, arXiv:1907.02257.

[70] U. Egede, T. Hurth, J. Matias, M. Ramon, and W. Reece, New physics reach of the decay mode $\bar{B} \rightarrow \bar{K}^{* 0} \ell^{+} \ell^{-}$, J. High Energy Phys. 10 (2010) 056.

[71] B. Aubert et al. (BABAR Collaboration), Direct $C P$, Lepton Flavor and Isospin Asymmetries in the Decays $B \rightarrow K^{(*)} \ell^{+} \ell^{-}$, Phys. Rev. Lett. 102, 091803 (2009).

[72] G. Hiller and F. Kruger, More model-independent analysis of $b \rightarrow s$ processes, Phys. Rev. D 69, 074020 (2004).

[73] R. N. Faustov and V. O. Galkin, Charmless weak $B_{s}$ decays in the relativistic quark model, Phys. Rev. D 87, 094028 (2013).

[74] A. Bharucha, D. M. Straub, and R. Zwicky, $B \rightarrow V \ell^{+} \ell^{-}$in the Standard Model from light-cone sum rules, J. High Energy Phys. 08 (2016) 098.

[75] A. Issadykov, $B_{s} \rightarrow K^{* 0}$ decay form factors from covariant confined quark model, EPJ Web Conf. 204, 08003 (2019).

[76] T. Kurimoto, H. N. Li, and A. I. Sanda, Leading power contributions to $B \rightarrow \pi, \rho$ transition form-factors, Phys. Rev. D 65, 014007 (2001).

[77] H. Y. Cheng, C. K. Chua, and C. W. Hwang, Covariant light-front approach for s-wave and p-wave mesons: its application to decay constants and form factors, Phys. Rev. D 69, 074025 (2004).

[78] W. Wang, Y. L. Shen, and C. D. Lü, Covariant light-front approach for $B_{c}$ transition form factors, Phys. Rev. D 79, 054012 (2009).

[79] Y. Y. Fan, Z. J. Xiao, R. M. Wang, and B. Z. Li, The $B \rightarrow$ $D^{(*)} l \nu_{l}$ decays in the pQCD approach with the Lattice QCD input, Sci. Bull. 60, 2009 (2015).

[80] X. Q. Hu, S. P. Jin, and Z. J. Xiao, Semileptonic decays $B_{c} \rightarrow\left(\eta_{c}, J / \psi\right) l \bar{\nu}_{l}$ in the "PQCD + Lattice" approach, Chin. Phys. C 44, 023104 (2020).

[81] X. Q. Hu, S. P. Jin, and Z. J. Xiao, Semileptonic decays $B / B_{s} \rightarrow\left(D^{(*)}, D_{s}^{(*)}\right) l \nu_{l}$ in the PQCD factorization approach with the lattice QCD input, Chin. Phys. C 44, 053102 (2020).

[82] J. M. Flynn, T. Izubuchi, T. Kawanai, C. Lehner, A. Soni, R. S. Van de Water, and O. Witzel, $B \rightarrow \pi \ell \nu$ and $B_{s} \rightarrow K \ell \nu$ form factors and $\left|V_{u b}\right|$ from $2+1$-flavor lattice QCD with domain-wall light quarks and relativistic heavy quarks, Phys. Rev. D 91, 074510 (2015).

[83] R. R. Horgan, Z. Liu, S. Meinel, and M. Wingate, Lattice QCD calculation of form factors describing the rare decays $B \rightarrow K^{*} \ell^{+} \ell^{-}$and $B_{s} \rightarrow \phi \ell^{+} \ell^{-}$, Phys. Rev. D 89, 094501 (2014).

[84] P. Ball and R. Zwicky, $B_{d, s} \rightarrow \rho, \omega, K^{*}, \phi$ decay formfactors from light-cone sum rules revisited, Phys. Rev. D 71, 014029 (2005). 
[85] D. Melikhov and B. Stech, Weak form-factors for heavy meson decays: An update, Phys. Rev. D 62, 014006 (2000).

[86] R. H. Li, C. D. Lu, and W. Wang, Transition form factors of B decays into p-wave axial-vector mesons in the perturbative QCD approach, Phys. Rev. D 79, 034014 (2009).

[87] C. D. Lu, W. Wang, and Z. T. Wei, Heavy-to-light form factors on the light cone, Phys. Rev. D 76, 014013 (2007).
[88] Y.L. Wu, M. Zhong, and Y.B. Zuo, $B_{s}, D_{s} \rightarrow \pi, K, \eta, \rho$, $K^{*}, \omega, \phi$ transition form factors and decay rates with extraction of the CKM parameters $\left|V_{u b}\right|,\left|V_{c s}\right|,\left|V_{c d}\right|$, Int. J. Mod. Phys. A 21, 6125 (2006).

[89] F. Su, Y. L. Wu, C. Zhuang, and Y. B. Yang, Charmless $B_{s} \rightarrow P P, P V, V V$ decays based on the six-quark effective Hamiltonian with strong phase effects II, Eur. Phys. J. C 72, 1914 (2012). 